\title{
Conjugacy in Garside groups I: cyclings, powers and rigidity
}

\author{
Joan S. Birman, ${ }^{1}$ Volker Gebhardt and Juan González-Meneses ${ }^{2}$
}

\begin{abstract}
In this paper a relation between iterated cyclings and iterated powers of elements in a Garside group is shown. This yields a characterization of elements in a Garside group having a rigid power, where 'rigid' means that the left normal form changes only in the obvious way under cycling and decycling. It is also shown that, given $X$ in a Garside group, if some power $X^{m}$ is conjugate to a rigid element, then $m$ can be bounded above by $\|\Delta\|^{3}$. In the particular case of braid groups $\left\{B_{n} ; n \in \mathbb{N}\right\}$, this implies that a pseudo-Anosov braid has a small power whose ultra summit set consists of rigid elements. This solves one of the problems in the way of a polynomial solution to the conjugacy decision problem (CDP) and the conjugacy search problem (CSP) in braid groups. In addition to proving the rigidity theorem, it will be shown how this paper fits into the authors' program for finding a polynomial algorithm to the $\mathrm{CDP} / \mathrm{CSP}$, and what remains to be done.
\end{abstract}

Mathematics Subject Classification (2000). 20F36, 20F10, 20E45.

Keywords. Garside groups, conjugacy problem, ultra summit set, rigidity, stable ultra summit set.

\section{Contents}

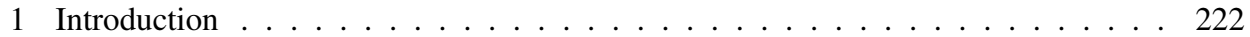

1.1 Garside groups . . . . . . . . . . . . . . . . . . . . . . 224

1.2 Solving the word and conjugacy problems in Garside groups . . . . . . . . . 228

1.3 The Thurston-Nielsen trichotomy in the braid groups . . . . . . . . . . . 237

1.4 A project to solve the conjugacy problems in braid groups and a summary of our results . . . . . . . . . . . . . . . . . 238

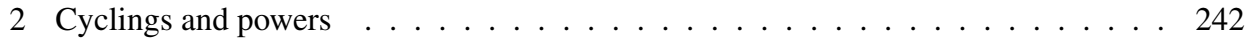

2.1 Decomposition of powers of $X \ldots \ldots \ldots \ldots \ldots \ldots \ldots \ldots$

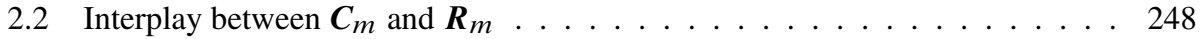

2.3 The absolute initial and final factors . . . . . . . . . . . . 251

2.4 The stable ultra summit set . . . . . . . . . . . . . . . . . . 257

\footnotetext{
${ }^{1}$ Partially supported by the U.S. National Science Foundation under Grants DMS-9973232 and DMS0405586.

${ }^{2}$ Partially supported by MTM2004-07203-C02-01 and FEDER.
} 


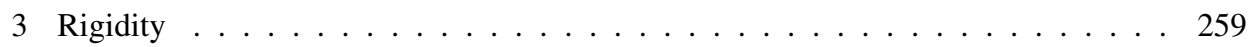

3.1 Rigidity of an element and behavior under cyclings and powers . . . . . . . . 259

3.2 The ultra summit set of a rigid element is made of rigid elements . . . . . . . 265

3.3 Elements having a rigid power . . . . . . . . . . . . . . . 267

3.4 Consequences for pseudo-Anosov braids . . . . . . . . . . . . . . . 270

3.5 A bound for the rigid power of an element . . . . . . . . . . . . 271

\section{Introduction}

Braid groups $B_{n}, n=1,2,3, \ldots$, were introduced in a foundational paper by Emil Artin [3] in 1925. In it Artin gave the well-known presentation:

$$
B_{n}=\left\langle\begin{array}{l|l}
\sigma_{1}, \ldots, \sigma_{n-1} \mid \begin{array}{ll}
\sigma_{i} \sigma_{j}=\sigma_{j} \sigma_{i} & \text { if }|i-j|>1, \\
\sigma_{i} \sigma_{j} \sigma_{i}=\sigma_{j} \sigma_{i} \sigma_{j} & \text { if }|i-j|=1
\end{array}
\end{array}\right\}
$$

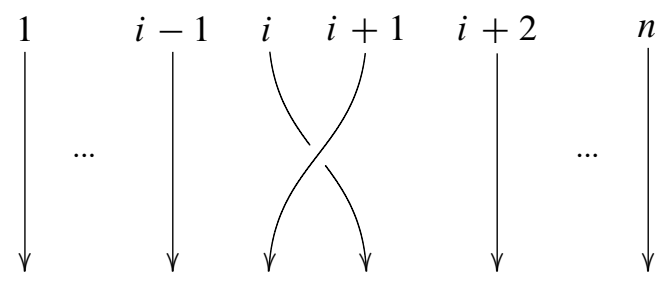

Figure 1. The elementary braid $\sigma_{i}$.

The elementary braid $\sigma_{i}$ is depicted in Figure 1 . To study $B_{n}$, Artin used the fact that there is a canonical homomorphism $\pi: B_{n} \rightarrow \Sigma_{n}$, where the image is the symmetric group, defined by sending a braid to the associated permutation of its endpoints. He went on to uncover the structure of the kernel of $\pi$, and used what he learned to solve the word problem in $B_{n}$ : to decide, for arbitrary words $X, Y$ in the generators and their inverses, whether they represent the same element of $B_{n}$. Artin also posed the conjugacy decision problem (CDP): to decide whether, for arbitrary $X, Y \in B_{n}$, there exists $Z \in B_{n}$ such that $Y=Z^{-1} X Z$. A different but related problem, the conjugacy search problem (CSP) asks to find $Z$, provided that one knows that it exists.

During the period 1925-1969 various efforts were made to solve the conjugacy problem, building on techniques which had been introduced in [3], but there was no significant progress. Then, in 1969 F. Garside [23] brought completely new techniques to bear, looking at $B_{n}$ in a very new way which stressed the similarity of its combinatorics to those of $\Sigma_{n}$, rather than focusing on $\operatorname{ker}\left(\pi: B_{n} \rightarrow \Sigma_{n}\right)$. Garside succeeded in solving both the word and conjugacy search problems simultaneously, and in a unified way. His methods were soon shown to apply to other groups too [14], 
[18], and over the years broadened to an entire class of groups which subsequently became known as Garside groups. The ideas that Garside introduced, and their subsequent improvements, are the subject of this paper, which is the first in a series with the unifying title 'Conjugacy in Garside groups I, II, III, ... They have a common goal: to improve Garside's algorithm for the CDP/CSP in a Garside group to obtain, in the particular case of the braid group $B_{n}$, an algorithm which is polynomial both in $n$ and an appropriate measure $\|X\|,\|Y\|$ of the complexity of $X$ and $Y$. This would have implications as regards the security of certain codes in public key cryptography [2], [28].

The existence of such a polynomial algorithm for the word problem in all Garside groups is now known via the work of [20], [21] for the braid groups. In [17] and [16] the class of Garside groups is defined in a more general setting, and shown to be biautomatic. It is a consequence of the way the definitions were chosen in [17], [16] that in fact, Garside's algorithm solves the word and CDP/CSP's in all Garside groups. Our work in this paper is a step in a program that we have developed to prove that the CDP/CSP in $B_{n}$ is polynomial in both $n$ and $\|X\|$. But all results in this paper are valid in every Garside group, except the results in $\$ 3.4$ and Theorem 3.37, where we consider applications of these results to the special case of braid groups, in particular to pseudo-Anosov braids.

Before we can state exactly what we do in this paper, and describe it in context, we need to set up necessary notation and review the known results and techniques. The combinatorial structure that we will use, and the new structure that we have uncovered, is quite complicated and, we think, interesting. In order to make this paper accessible to non-experts we give details and examples which those who are acquainted with the literature will probably wish to bypass quickly, moving on to $\$ 1.4$, where we describe the essential content of this paper and its context in our larger goal, and thence to $\S 2$, where our new contributions begin.

Acknowledgements. J. Birman and J. González-Meneses, who were working together, and V. Gebhardt, became acquainted with each other's partial results at a conference in the Banff International Research Station for Mathematical Innovation and Discovery, in October 2004. There was some overlap, and also some recognition that differing viewpoints could lead to progress, so they decided to pool forces at that time. The three authors thank the PIMS, MSRI, MITACS and IM-UNAM for their wisdom in sponsoring international conferences which foster exactly this kind of fruitful interchange and collaboration.

J. Birman thanks the Project MTM2004-07203-C02-01 of the Spanish Ministerio de Ciencia y Tecnología for hosting her visit to Seville in November 2004, so that she and J. González-Meneses could work together on this project.

J. González-Meneses thanks the project MTM2004-07203-C02-01 and the Department of Mathematics of Columbia University for hosting his two visits to New York, in July 2004 and March-April 2006. 
V. Gebhardt thanks the Department of Algebra of the University of Seville, and the Junta de Andalucía, for funding his visit to Seville in January 2006.

The work in this paper and [9], [10] was done simultaneously and independently from the work of S. J. Lee and E. K. Lee in [30], [31], [32]. We first became aware of that work when we were in the process of writing up this one. Some of this work, notably Proposition 2.23 below and the results in [9], were reported on in talks at conferences in Banff in October 2004 and Luminy in June 2005.

1.1. Garside groups. Among the known equivalent definitions of Garside groups, we use the one which was suggested to us by John Crisp [15], because it seems the most natural of the many possible definitions. A group $G$ is said to be a Garside group if it satisfies properties (A), (B) and (C) below:

(A) $G$ admits a lattice order $(G, \preccurlyeq, \vee, \wedge)$, invariant under left-multiplication.

This means that there is a partial order $\preccurlyeq$ on the elements of $G$ such that $a \preccurlyeq b$ implies $c a \preccurlyeq c b$ for every $c \in G$. Also, every pair of elements $s, t \in G$ admits a unique $\operatorname{lcm} s \vee t$ and a unique ged $s \wedge t$ with respect to $\preccurlyeq$. This partial order $\preccurlyeq$ defines a submonoid $P \subset G$, called the positive cone of $G$, defined by $P=\{p \in G ; 1 \preccurlyeq p\}$. Notice that the invariance of $\preccurlyeq$ under left-multiplication implies that $P \cap P^{-1}=\{1\}$, and also that $a \preccurlyeq b \Longleftrightarrow a^{-1} b \in P$. Hence the submonoid $P$ determines the partial order $\preccurlyeq$, so we shall equally talk about the lattice $(G, P)$. We remark that if $a, b \in P$ then $a \preccurlyeq b$ if and only if $a$ is a prefix of $b$, that is, there exists $c \in P$ such that $a c=b$. This is why $\preccurlyeq$ is sometimes called the prefix order.

There is also a related suffix order, defined by $b \succcurlyeq a$ if $b a^{-1} \in P$. It is important that $a \preccurlyeq b$ does not imply that $b \succcurlyeq a$. Sometimes we will get genuinely new information by using both orderings, even when the proofs are little more than copies of one-another.

(B) There exists an element $\Delta \in P$, called the Garside element, satisfying:

(a) The interval $[1, \Delta]=\{s \in G ; 1 \preccurlyeq s \preccurlyeq \Delta\}$ generates $G$. Its elements are called the simple elements of $G$. We shall always assume that $[1, \Delta]$ is finite, that is, that $G$ has finite type.

(b) Conjugation by $\Delta$ preserves the positive cone $P: \Delta^{-1} P \Delta=P$.

We remark that if $\Delta$ satisfies both (a) and (b), then $[1, \Delta]$ also generates $P$ as a monoid, which is one of the properties usually required in the definition of a Garside element.

(C) The monoid $P$ is atomic.

This means that for every $x \in P$ there exists an upper bound on the length of a (strict) chain $1 \prec x_{1} \prec \cdots \prec x_{r}=x$. In other words, if we define the atoms of $G$ as the elements $a \in P$ which cannot be decomposed in $P$ (there are no 
nontrivial $b, c \in P$ such that $a=b c$ ), then for every $x \in P$ there exists an upper bound on the number of atoms in a product $x=a_{1} a_{2} \ldots a_{r}$ with each $a_{i}$ an atom. In particular, if $P$ is atomic, one can define the length of an element $x \in P$ as the maximal length of such a chain, that is,

$$
\|x\|=\max \left\{n ; x=a_{1} a_{2} \ldots a_{n}, \text { where } a_{i} \in P \backslash\{1\}\right\} .
$$

Notice that the atoms generate $G$.

These data determine a Garside structure on $G$, which may be defined as follows: Let $G$ be a countable group, $P$ be a submonoid, and $\Delta \in P$. The triple $(G, P, \Delta)$ is said to be a (finite type) Garside structure on $G$ if $(G, P)$ is a lattice, $\Delta$ is a Garside element (with $[1, \Delta]$ finite), and $P$ is atomic. We remark that a given group $G$ may admit more than one Garside structure.

Example 1. Our first example is very simple. We consider the braid group $B_{3}$ and its two known Garside structures:

1A. The classical Garside structure is associated to the presentation (1) of $B_{3}$. The Garside element is $\sigma_{1} \sigma_{2} \sigma_{1}=\sigma_{2} \sigma_{1} \sigma_{2}$. The elements in $P$ correspond to the braids in which all crossings are positive. The atoms are $\sigma_{1}$ and $\sigma_{2}$.

1B. If we set $x=\sigma_{1}, y=\sigma_{2}$ and $z=\sigma_{2} \sigma_{1} \sigma_{2}^{-1}$ we get the presentation $\langle x, y, z \mid x y=y z=z x\rangle$. The Garside element is now $\Delta=x y$. See [39] for the way in which this structure was used to solve the shortest word problem in $B_{3}$ and to give an algorithm for determining the genus of knots and links which are closed 3-braids. This Garside structure was generalized to all $n$ in [11].

Example 2 (Free abelian groups of finite rank). This is another very simple example of a Garside group:

$$
\mathbb{Z}^{n}=\left\langle x_{1}, \ldots, x_{n} \mid x_{i} x_{j}=x_{j} x_{i}, i<j\right\rangle .
$$

The positive cone is

$$
\mathbb{N}^{n}=\left\{x_{1}^{k_{1}} \ldots x_{n}^{k_{n}} ; k_{i} \geq 0 \text { for all } i\right\} .
$$

The Garside element is $\Delta=x_{1} \ldots x_{n}$, and the simple elements have the form $x_{1}^{k_{1}} \ldots x_{n}^{k_{n}}$ where $k_{i} \in\{0,1\}$ for every $i=1, \ldots, n$. Hence there are $2^{n}$ simple elements.

Example 3 (The braid group $B_{n}$, with the classical Garside structure). Garside used the presentation (1). The usual Garside structure in this group is determined by $\left(B_{n}, B_{n}^{+}, \Delta\right)$, where $B_{n}^{+}$is the monoid of positive braids, consisting of the elements in $B_{n}$ that can be written as a product of $\sigma_{i}$ 's with no $\sigma_{i}^{-1}$, and

$$
\Delta=\left(\sigma_{1}\right)\left(\sigma_{2} \sigma_{1}\right)\left(\sigma_{3} \sigma_{2} \sigma_{1}\right) \ldots\left(\sigma_{n-1} \ldots \sigma_{1}\right)
$$


is a half-twist on all of the strands. The atoms are $\sigma_{1}, \ldots, \sigma_{n-1}$. The elements in $P$ correspond to the braids in which all crossings are positive. The Garside element $\Delta$ can be characterized as the only positive braid in which every pair of strands cross exactly once. The simple elements, in the case of $B_{n}$, are the positive braids in which every pair of strands cross at most once. It follows that every simple element corresponds to a permutation on the set of $n$ elements (the strands). Hence there are $n$ ! simple elements in $B_{n}^{+}$, with this Garside structure. Figure 2 shows the Hasse diagram representing the lattice of simple elements in $B_{4}^{+}$. In this diagram, an element $a$ is joined by a line to an element $b$ in the upper row if and only if $a \preccurlyeq b$. Moreover, each line type corresponds to a right multiplication by an atom: a single line corresponds to $\sigma_{1}$, a double line to $\sigma_{2}$ and a dotted line to $\sigma_{3}$. This lattice of simple elements determines the whole Garside structure of the group.

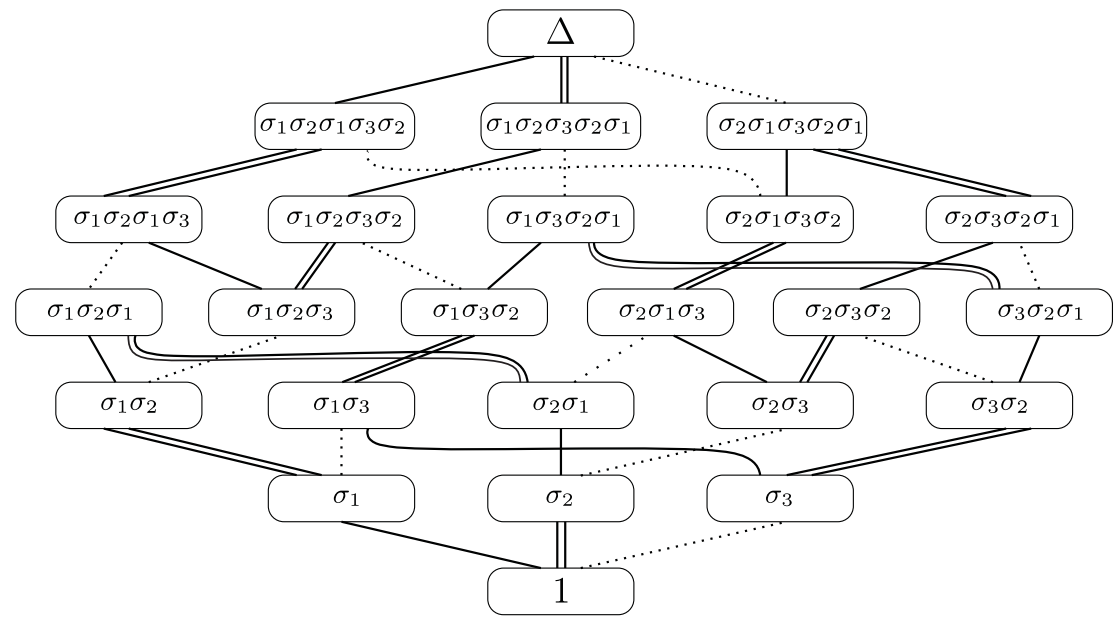

Figure 2. The lattice of simple elements in $B_{4}^{+}$. There are $4 !=24$ elements.

Example 4 (Spherical type Artin-Tits groups [13]). The previous three examples were particular cases of Artin-Tits groups. All Artin-Tits groups of spherical type are known to be Garside groups [14]. Given a finite set $S$, a Coxeter matrix over $S$ is a symmetric matrix $M=\left(m_{s t}\right)_{s, t \in S}$, where $m_{s s}=1$ for all $s \in S$ and $m_{s, t} \in$ $\{2,3, \ldots, \infty\}$. Every Coxeter matrix $M$ defines a group $A_{M}$ given by the following presentation:

$$
A_{M}=\langle S| \underbrace{s t s t \ldots}_{m_{s t} \text { terms }}=\underbrace{t s t s \ldots}_{m_{s t} \text { terms }}, \text { for all } s, t \in S\rangle,
$$

where $m_{s t}=\infty$ means that there is no relation involving $s$ and $t$. The group $A_{M}$ is called the Artin-Tits group associated to $M$, also called Artin group or generalized braid group. 
If one adds to the above presentation the relations $s^{2}=1$ for all $s \in S$, one obtains the group $W_{M}$, called the Coxeter group associated to $M$. An Artin-Tits group is said to be of spherical type if its corresponding Coxeter group is finite.

The usual Garside structure in these groups is given by $\left(A_{M}, A_{M}^{+}, \Delta\right)$, where $A_{M}^{+}$is the monoid of positive elements, consisting of products of elements of $S$ (the above presentation of $A_{M}$, considered as a monoid presentation, gives $A_{M}^{+}$), and the Garside element $\Delta$ is defined as follows. The set of generators $S$ can be decomposed into two sets $S=S_{1} \cup S_{2}$, where elements contained in the same set $S_{i}$ commute. This decomposition can be easily obtained from the Coxeter graph of the group. The reader is referred to [13] for the definition of a Coxeter graph and its associated Coxeter matrix, and also for a list of the Coxeter graphs associated to the finite Coxeter groups. If the Coxeter graph $\Gamma$ is connected, there is only one possible decomposition of $S$ in the above sense. Now define

$$
\Delta_{1}=\prod_{s \in S_{1}} s \quad \text { and } \quad \Delta_{2}=\prod_{s \in S_{2}} s
$$

Then one has

$$
\Delta=\underbrace{\Delta_{1} \Delta_{2} \Delta_{1} \Delta_{2} \ldots}_{h \text { terms }}
$$

where $h$ is the Coxeter number of the corresponding Coxeter group. The Coxeter numbers corresponding to the spherical type Artin-Tits groups are given it the following table:

\begin{tabular}{|c||c|c|c|c|c|c|c|c|c|c|c|}
\hline Type & $A_{l}$ & $B_{l}$ & $D_{l}$ & $E_{6}$ & $E_{7}$ & $E_{8}$ & $F_{4}$ & $G_{2}$ & $H_{3}$ & $H_{4}$ & $I_{2}(p)$ \\
\hline$h$ & $l+1$ & $2 l$ & $2 l-2$ & 12 & 18 & 30 & 12 & 6 & 10 & 30 & $p$ \\
\hline
\end{tabular}

As an example, the Garside element of the spherical Artin-Tits group of type $B_{l}$ is $\Delta=\left(\left(s_{1} s_{3} \ldots s_{l}\right)\left(s_{2} s_{4} \ldots s_{l-1}\right)\right)^{l}$ when $l$ is odd, and $\Delta=\left(\left(s_{1} s_{3} \ldots s_{l-1}\right)\right.$ $\left.\left(s_{2} s_{4} \ldots s_{l}\right)\right)^{l}$ when $l$ is even. Notice that the Artin-Tits monoid of type $A_{l}$ is precisely the Artin braid monoid on $l+1$ strands. Notice also that the Garside structure given by this construction coincides with the original Artin structure for braid groups described above.

We remark that every spherical type Artin-Tits group admits another Garside structure, discovered in [6], called the dual Garside structure. In the case of braid groups, the dual Garside structure is precisely the one discovered in [11].

Example 5 (Torus knot groups). The fundamental group of the complement of a $(p, q)$-torus knot, where $p, q>1$ are coprime, is given by the following presentation:

$$
\left\langle x, y \mid x^{p}=y^{q}\right\rangle \text {. }
$$

If we consider the monoid given by this presentation, it is a Garside monoid with Garside element $\Delta=x^{p}$. 
Example 6. The following two examples of Garside groups do not belong to a class of known groups, but they have interesting properties which are not satisfied by the groups in the previous examples. They were discovered and studied by Picantin in [36]. In both cases, we give presentations of the groups which, considered as monoid presentations, yield the corresponding Garside monoids. Hence we shall only define the Garside element, in each case.

(1) $G=\left\langle x, y, z \mid x z x y=y z x^{2}, y z x^{2} z=z x y z x=x z x y z\right\rangle$. The Garside element is $\Delta=x z x y z x$. In most examples of Garside groups, the Garside element $\Delta$ is the least common multiple (with respect to $\preccurlyeq$ ) of the atoms. In this example, since the relations are homogeneous, the atoms are just the letters $x, y$ and $z$, and one has $\operatorname{lcm}_{\preccurlyeq}(x, y, z)=x z x y z$. But $x z x y z \neq \Delta$. Indeed, since conjugation by $\Delta$ must preserve the set of atoms, all atoms must be left and right divisors of $\Delta$, but we have $x z x y z \nsucceq y$, hence $\operatorname{lcm}_{\preccurlyeq}(x, y, z)=x z x y z \neq$ $\mathrm{lcm}_{\succcurlyeq}(x, y, z)$. This is an example of a Garside monoid in which the $1 \mathrm{~cm} \preccurlyeq$ of the atoms is not a Garside element.

(2) $G=\left\langle x, y \mid \operatorname{xy} x y x=y^{2}\right\rangle$. Garside element $\Delta=y^{3}$. In this case, $\mathrm{lcm}_{\preccurlyeq}(x, y)=\operatorname{lcm}_{\succcurlyeq}(x, y)=y^{2}$, but $y^{2}$ is not a Garside element. Indeed, since conjugation by $\Delta$ must preserve the set of simple elements, the set of positive left-divisors and the set of positive right-divisors of $\Delta$ must coincide. But this does not happen for $y^{2}$. For instance $x y x y \preccurlyeq y^{2}$ but $y^{2} \neq x y x y$. This is also an example of a Garside monoid in which the relations are not homogeneous, hence the length of a positive element is not given by the letter length of any representative.

Construction of new Garside monoids. We already provided several examples of Garside monoids and groups. Using these monoids as building blocks, one can construct new Garside monoids and groups thanks to the following result. In [36] there is a definition of the so called crossed product of monoids, which also allows to construct new Garside monoids.

Theorem 1.1 ([36]). The crossed product of Garside monoids is a Garside monoid.

An example of crossed product, given in [33], is the semidirect product $\mathbb{Z} \ltimes G^{n}$, where the action of $\mathbb{Z}$ on the free product $G^{n}$ ( $G$ is a Garside group) is given by cyclic permutations of coordinates.

1.2. Solving the word and conjugacy problems in Garside groups. From now on, we will fix a Garside group $G$ with a finite type Garside structure $(G, P, \Delta)$. We will show how to solve the word problem, giving a well known normal form for elements in a Garside group. The basic reference is [20]. While everything in that paper relates to the braid groups, most of it generalizes easily to arbitrary Garside groups, which were singled out as a class several years later [17], [16]. 
Definition 1.2 (Left normal form). Given $X \in G$, we will say that a decomposition $X=\Delta^{p} x_{1} \ldots x_{r}(r \geq 0)$ is the left normal form of $X$ is it satisfies

(1) $p \in \mathbb{Z}$ is maximal such that $\Delta^{p} \preccurlyeq X$, that is, $x_{1} \ldots x_{r} \in P$ and $\Delta \nless x_{1} \ldots x_{r}$;

(2) $x_{i}=\left(x_{i} \ldots x_{r}\right) \wedge \Delta$, for $i=1, \ldots, r$. That is, $x_{i}$ is the biggest simple prefix of $x_{i} \ldots x_{r}$.

One can also show by induction that $\Delta^{p} x_{1} \ldots x_{i}=X \wedge \Delta^{p+i}$, for $i=1, \ldots, r$.

It is known that one can check whether a given decomposition $X=\Delta^{p} x_{1} \ldots x_{r}$ is a left normal form by looking at each pair of consecutive factors $x_{i} x_{i+1}$. We say that a pair of simple elements $a, b \in[1, \Delta]$ is left weighted if the product $a b$ is in left normal form as written, that is, if $a=(a b) \wedge \Delta$. Then $\Delta^{p} x_{1} \ldots x_{r}$ is a left normal form if and only if $x_{1} \neq \Delta$ and each pair $x_{i} x_{i+1}$ is left weighted.

Notice that if we consider the set of simple elements as a set of generators for $G$, then the decomposition defined above is a normal form in the usual sense, that is, a unique way to write any element of $G$ as a product of the generators and their inverses. If one wishes to obtain a normal form with respect to any other set of generators (for example the set of atoms), one just needs to choose a unique way to write each simple element in terms of the desired generators, and replace this in the left normal form.

We now give several standard terms that will be needed to work with Garside groups.

If $X=\Delta^{p} x_{1} \ldots x_{r}$ is in left normal form, the infimum, supremum and canonical length of $X$ are defined by $\inf (X)=p, \sup (X)=p+r$ and $\ell(X)=r$, respectively.

The shift map $\tau$ is the inner automorphism $\tau: G \rightarrow G$ given by $\tau(x)=\Delta^{-1} x \Delta$.

Given a simple element $x$, we define $x^{*}=x^{-1} \Delta$. That is, $x^{*}$ is the only simple element such that $x x^{*}=\Delta$, and is called the right complement of $x$. The element $x^{*}$ is the maximal element $s$ (with respect to $\preccurlyeq$ ) such that $x s$ is simple. A product $a b$ of simple elements $a$ and $b$ is left weighted if and only if $a^{*} \wedge b=1$. It will be convenient to define the right complement map $\partial:[1, \Delta] \rightarrow[1, \Delta]$ by $\partial(x)=x^{*}$ :

Lemma 1.3. The map $\partial:[1, \Delta] \rightarrow[1, \Delta]$ is a bijection, and $\partial^{2}=\tau$.

Proof. We show that $\partial$ is a bijection by defining its inverse $\partial^{-1}:[1, \Delta] \rightarrow[1, \Delta]$ as $\partial^{-1}(y)=\Delta y^{-1}$. The element $\Delta y^{-1}$, sometimes denoted ${ }^{*} y$, is called the left complement of $y$. It is the only simple element such that ${ }^{*} y y=\Delta$.

On the other hand, $\partial^{2}(x)=\partial\left(x^{-1} \Delta\right)=\left(\Delta^{-1} x\right) \Delta=\tau(x)$, as we wanted to show.

Corollary 1.4. There exists a positive integer e such that $\Delta^{e}$ belongs to the center of $G$. More precisely, one has $\tau([1, \Delta])=[1, \Delta]$ and $\tau(A)=A$, where $A$ is the set of atoms in $G$, and $\tau^{e}=\mathrm{id}_{G}$ for some positive integer $e$, so that $\Delta^{e}$ is central. 
Proof. Since $\partial([1, \Delta])=[1, \Delta]$, it follows that $\tau([1, \Delta])=\partial^{2}([1, \Delta])=[1, \Delta]$. This also implies that $\tau(A)=A$. Indeed, suppose that there is some atom $a$ such that $\tau(a)$ is not an atom. Then $\tau(a)$ is a simple element that can be decomposed into a product of two simple elements $\tau(a)=s t$. But then $\tau^{-1}(s)$ and $\tau^{-1}(t)$ are simple elements such that $a=\tau^{-1}(\tau(a))=\tau^{-1}(s) \tau^{-1}(t)$. A contradiction, since $a$ is an atom. Hence $\tau(A) \subset A$. Since $A$ is a finite set, and $\tau: G \rightarrow G$ is a bijection, it follows that $\tau(A)=A$.

Finally, since $\tau$ induces a permutation in $A$, there exists a positive integer $e$ such that $\tau^{e}$ induces the trivial permutation on $A$. Since the atoms generate $G$, it follows that $\tau^{e}$ is the trivial automorphism of $G$. That is to say, $\Delta^{e}$ is central.

Remark. In the braid group $B_{n}$ one has $e=2$, so $\Delta^{2}$ is central. Furthermore, the center of $B_{n}$ is the cyclic group generated by $\Delta^{2}$.

The right complement plays an important role when comparing the left normal forms of $X$ and $X^{-1}$.

Theorem 1.5 ([20]). If

$$
X=\Delta^{p} x_{1} \ldots x_{r}
$$

in left normal form, then the left normal form of $X^{-1}$ is equal to

$$
X^{-1}=\Delta^{-p-r} x_{r}^{\prime} \ldots x_{1}^{\prime},
$$

where $x_{i}^{\prime}=\tau^{-p-i}\left(\partial\left(x_{i}\right)\right)$ for $i=1, \ldots, r$.

Remark. Notice that $x_{i}^{\prime}=\tau^{-p-i}\left(\partial\left(x_{i}\right)\right)=\partial^{-2 p-2 i+1}\left(x_{i}\right)$, so the left normal form of $X^{-1}$ is equal to

$$
X^{-1}=\Delta^{-p-r} \partial^{-2 p-2 r+1}\left(x_{r}\right) \partial^{-2 p-2 r+3}\left(x_{r-1}\right) \ldots \partial^{-2 p-1}\left(x_{1}\right) .
$$

Corollary 1.6. For every $X \in G$, one has $\inf \left(X^{-1}\right)=-\sup (X), \sup \left(X^{-1}\right)=$ $-\inf (X)$ and $\ell\left(X^{-1}\right)=\ell(X)$.

See Section 9.5 of [21] for a proof that an $n$-braid of length $m$ can be put in left normal form in running time $O\left(m^{2} n \log n\right)$, with the usual Garside structure of $B_{n}$, and see [11] to find how one can compute the normal form in time $O\left(m^{2} n\right)$, using the dual Garside structure of $B_{n}$, usually known as Birman-Ko-Lee structure. In general, using the normal form algorithm, the complexity of computing the left normal form of a given element in a Garside group $G$ is $O\left(m^{2} p\right)$, where $p$ is the complexity of computing the gcd of two simple elements in $G$. The number $p$ usually depends on the length of $\Delta$ (simple elements are smaller than $\Delta$ ) and on the number of atoms in $G$, since one usually computes the gcd of two elements by iteratively testing if there is some atom which is a common prefix. 
We now explain the algorithms for solving the conjugacy decision and search problems (CDP/CSP) in Garside groups that were given in [23], [20], [22], [24]: given two elements $X, Y \in G$, determine if $X$ and $Y$ are conjugate and, if this is the case, compute a conjugating element $Z$ such that $X^{Z}=Z^{-1} X Z=Y$. Each algorithm in [23], [20], [22], [24] is an improvement of the previous one, but the basic idea is the same in all of them: Given an element $X \in G$, the algorithm computes a finite subset $I_{X}$ of the conjugacy class of $X$ which has the following properties:

(1) For every $X \in G$, the set $I_{X}$ is finite, non-empty and only depends on the conjugacy class of $X$. In particular, two elements $X, Y \in G$ are conjugate if and only if $I_{X}=I_{Y}$ or, equivalently, $I_{X} \cap I_{Y} \neq \emptyset$.

(2) Given $X \in G$, a representative $\tilde{X} \in I_{X}$ and an element $a \in G$ such that $X^{a}=\tilde{X}$ can be computed effectively.

(3) Given a non-empty subset $I \subset I_{X}$, there is a finite process which either proves that $I=I_{X}$ or produces an element $Z \in I$ and an element $b \in G$ such that $Z^{b} \in I_{X} \backslash I$. In particular, $I_{X}$ can be constructed from any representative as the closure under this process.

Given $X, Y \in G$, solving the CDP/CSP then involves the following steps.

(a) Find representatives $\tilde{X} \in I_{X}$ and $\tilde{Y} \in I_{Y}$.

(b) Repeatedly use the process from (3), keeping track of the conjugating elements, to compute further elements of $I_{X}$ until either

(i) $\tilde{Y}$ is found as an element of $I_{X}$, proving $X$ and $Y$ to be conjugate and providing a conjugating element, or

(ii) the entire set $I_{X}$ has been constructed without encountering $\tilde{Y}$, proving that $X$ and $Y$ are not conjugate.

We now discuss, briefly, each particular algorithm in [23], [20], [22], [24]. In Garside's original algorithm [23], the set $I_{X}$ is the summit set of $X$, denoted $\operatorname{SS}(X)$, which is the set of conjugates of $X$ having maximal infimum. This was improved by ElRifai and Morton [20] who considered $I_{X}=\operatorname{SSS}(X)$, the super summit set of $X$, consisting of the conjugates of $X$ having minimal canonical length. They also show that $\operatorname{SSS}(X)$ is the set of conjugates of $X$ having maximal infimum and minimal supremum, at the same time.

For instance, in the braid group $B_{n}$ with the usual Garside structure, one has $\operatorname{SS}\left(\sigma_{1}\right)=\operatorname{SSS}\left(\sigma_{1}\right)=\left\{\sigma_{1}, \ldots, \sigma_{n-1}\right\}$. A small example in which $\operatorname{SSS}(X)$ is strictly smaller than $\operatorname{SS}(X)$ is given by $X=\Delta \sigma_{1} \sigma_{1} \in B_{3}$, for which $\operatorname{SSS}(X)=\left\{\Delta \cdot \sigma_{1} \sigma_{3}\right\}$ and $\operatorname{SS}(X)=\left\{\Delta \cdot \sigma_{1} \sigma_{3}, \Delta \cdot \sigma_{1} \cdot \sigma_{1}, \Delta \cdot \sigma_{3} \cdot \sigma_{3}\right\}$ (the factors in each left normal form are separated by a dot). In general $\operatorname{SSS}(X)$ is much smaller than $\operatorname{SS}(X)$.

Starting by a given element $X$, one can find an element $\tilde{X} \in \operatorname{SSS}(X)$ by a sequence of special conjugations, called cyclings and decyclings. The conjugating elements 
involved in a cycling or a decycling will play a crucial role later, so we start by defining them.

Definition 1.7. Given $X \in G$ whose left normal form is $X=\Delta^{p} x_{1} \ldots x_{r}(r>0)$, we define the initial factor of $X$ as $\iota(X)=\tau^{-p}\left(x_{1}\right)$, and the final factor of $X$ as $\varphi(X)=x_{r}$. If $r=0$ we define $\iota\left(\Delta^{p}\right)=1$ and $\varphi\left(\Delta^{p}\right)=\Delta$.

Remark. Up to conjugation by $\Delta^{p}$, the simple element $\iota(X)(\operatorname{resp} . \varphi(X))$ corresponds to the first (resp. last) non- $\Delta$ factor in the left normal form of $X$. An equivalent definition of $\iota(X)$ and $\varphi(X)$, which does not involve the left normal form of $X$ (although it involves its infimum and supremum), is the following.

- $\iota(X)=X \Delta^{-p} \wedge \Delta$.

- $\varphi(X)=\left(\Delta^{p+r-1} \wedge X\right)^{-1} X$.

This explains why $\iota\left(\Delta^{p}\right)$ and $\varphi\left(\Delta^{p}\right)$ are defined in the above way.

The initial and final factors of $X$ and $X^{-1}$ are closely related.

Lemma 1.8. For every $X \in G$ onehas $\iota\left(X^{-1}\right)=\partial(\varphi(X))$ and $\varphi\left(X^{-1}\right)=\partial^{-1}(\iota(X))$.

Proof. Let $\Delta^{p} x_{1} \ldots x_{r}$ be the left normal form of $X$, and suppose that $r>0$. We know that $\Delta^{-p-r} x_{r}^{\prime} \ldots x_{1}^{\prime}$ is the left normal form of $X^{-1}$, where $x_{i}^{\prime}=\tau^{-p-i}\left(\partial\left(x_{i}\right)\right)$. Hence one has $\iota\left(X^{-1}\right)=\tau^{p+r}\left(x_{r}^{\prime}\right)=\tau^{p+r}\left(\tau^{-p-r}\left(\partial\left(x_{r}\right)\right)\right)=\partial\left(x_{r}\right)=\partial(\varphi(X))$. Permuting $X$ and $X^{-1}$ in this formula yields $\iota(X)=\partial\left(\varphi\left(X^{-1}\right)\right)$, hence $\varphi\left(X^{-1}\right)=$ $\partial^{-1}(\iota(X))$.

If $r=0$, that is if $X=\Delta^{p}$, then $\iota\left(X^{-1}\right)=1=\partial(\Delta)=\partial(\varphi(X))$, and $\varphi\left(X^{-1}\right)=\Delta=\partial^{-1}(1)=\partial^{-1}(\iota(X))$, so the result is also true in this case.

Remark. The above result can be restated as follows: For every $X \in G$, one has $\varphi(X) \iota\left(X^{-1}\right)=\Delta=\varphi\left(X^{-1}\right) \iota(X)$.

We can now define the very special conjugations called cyclings and decyclings.

Definition 1.9. Given $X \in G$, we call $\mathbf{c}(X)=X^{\iota(X)}$ the cycling of $X$ and we call $\mathbf{d}(X)=X^{\varphi(X)^{-1}}$ the decycling of $X$. In other words, if $\Delta^{p} x_{1} \ldots x_{r}$ is the left normal form of $X$ and $r>0$, then

$$
\mathbf{c}(X)=\Delta^{p} x_{2} \ldots x_{r} \tau^{-p}\left(x_{1}\right) \quad \text { and } \quad \mathbf{d}(X)=x_{r} \Delta^{p} x_{1} \ldots x_{r-1} .
$$

In the case $\ell(X)=0$, we have $\mathbf{c}(X)=\mathbf{d}(X)=X$.

Roughly speaking, for an element of positive canonical length, the cycling of $X$ is computed by passing the first simple factor of $X$ to the end, while the decycling of $X$ is 
computed by passing the last simple factor of $X$ to the front. However, the powers of $\Delta$ are not taken into account, which is why one must use the automorphism $\tau$. Notice that the above decompositions of $\mathbf{c}(X)$ and $\mathbf{d}(X)$ are not, in general, left normal forms. Hence, if one wants to perform iterated cyclings or decyclings, one needs to compute the left normal form of the resulting element at each iteration.

As we said above, cyclings and decyclings can be used to find an element in $\operatorname{SSS}(X)$, given $X$. The following result was shown for braid groups, but the same proof is valid for every Garside group.

Theorem 1.10 ([20], [12]). Let $(G, P, \Delta)$ be a Garside structure offinite type. Choose $X \in G$, and let $r=\ell(X)$. Let $m$ be the letter length of $\Delta$.

(1) A sequence of at most rm cyclings and decyclings applied to $X$ produces $a$ representative $\tilde{X} \in \operatorname{SSS}(X)$.

(2) If $Y \in \operatorname{SSS}(X)$ and $\alpha \in P$ is such that $Y^{\alpha} \in \operatorname{SSS}(X)$ then $Y^{\alpha \wedge \Delta} \in \operatorname{SSS}(X)$.

Notice that $\alpha \wedge \Delta$ is always a simple element. Since the set of simple elements is finite, one has the following:

Corollary 1.11 ([20]). Let $X \in G$ and $\mathcal{V} \subset \operatorname{SSS}(X)$ be non-empty. If $\mathcal{V} \neq \operatorname{SSS}(X)$ then there exist $Y \in \mathcal{V}$ and a simple element such that $Y^{s} \in \operatorname{SSS}(X) \backslash \mathcal{V}$.

Since $\operatorname{SSS}(X)$ is a finite set, the above corollary allows to compute the whole $\operatorname{SSS}(X)$. More precisely, if one knows a subset $\mathcal{V} \subset \operatorname{SSS}(X)$ (at the beginning $\mathcal{V}=\{\tilde{X}\}$ ), one conjugates each element in $\mathcal{V}$ by all simple elements (recall that $G$ is of finite type, that is, the set of simple elements is finite). If one encounters a new element $Z$ with the same canonical length as $\tilde{X}$ (a new element in $\operatorname{SSS}(X)$ ), then consider $\mathcal{V} \cup\{Z\}$ and start again. If no new element is found, this means that $\mathcal{V}=\operatorname{SSS}(X)$, and we are done. One important remark is that this algorithm not only computes the set $\operatorname{SSS}(X)$, but it also provides conjugating elements joining the elements in SSS $(X)$. Hence it solves both the CDP and the CSP in Garside groups.

The computational cost of computing $\operatorname{SSS}(X)$ depends mainly on two factors: the size of $\operatorname{SSS}(X)$ and the number of simple elements. If we consider braid groups $B_{n}$ with the usual Garside structure, for instance, all known upper bounds for the size of $\operatorname{SSS}(X)$ are exponential in $n$, although it is conjectured that for fixed $n$ a polynomial bound in the canonical length of $X$ exists [21]. Recall also that the number of simple elements is $n$ !, and one needs to conjugate every element in $\operatorname{SSS}(X)$ by all simple elements. Fortunately, this task can be avoided thanks to the following result.

Theorem 1.12 ([22]). Let $X \in \operatorname{SSS}(X)$. If $s, t \in G$ are such that $X^{s} \in \operatorname{SSS}(X)$ and $X^{t} \in \operatorname{SSS}(X)$, then $X^{s \wedge t} \in \operatorname{SSS}(X)$. 
Corollary 1.13. Let $X \in G$ and $Y \in \operatorname{SSS}(X)$. For every $u \in P$ there is a unique $\preccurlyeq$-minimal element $\rho_{Y}(u)$ satisfying

$$
u \preccurlyeq \rho_{Y}(u) \text { and } Y^{\rho_{Y}(u)} \in \operatorname{SSS}(X) .
$$

Proof. The gcd of $\left\{v \in P ; u \leqslant v, Y^{v} \in \operatorname{SSS}(X)\right\}$ is the element $\rho_{Y}(u)$ and has all the claimed properties.

The set $\rho_{Y}(A)=\left\{\rho_{Y}(a) ; a\right.$ is an atom $\}$ contains all nontrivial elements which are $\preccurlyeq$-minimal among those conjugating $Y$ to an element in $\operatorname{SSS}(X)$. We call the latter the minimal simple elements for $Y$ with respect to $\operatorname{SSS}(X)$. Since one could have $\rho_{Y}(a) \prec \rho_{Y}(b)$ (strict) for two distinct atoms $a$ and $b$, the set of minimal simple elements for $Y$ is in general strictly contained in $\rho_{Y}(A)$.

Corollary 1.14. Let $X \in G$ and $\mathcal{V} \subset \operatorname{SSS}(X)$ be non-empty. If $\mathcal{V} \neq \operatorname{SSS}(X)$ then there exist $Y \in \mathcal{V}$ and a minimal simple element $\rho=\rho_{Y}(a)$ for some atom a such that $Y^{\rho} \in \operatorname{SSS}(X) \backslash \mathcal{V}$.

Using the technique of minimal simple elements, super summit sets can be computed as in [20], but instead of conjugating each element $Y \in \operatorname{SSS}(X)$ by all simple elements, it suffices to conjugate $Y$ by its minimal simple elements. Notice that the number of minimal simple elements for a given $Y \in \operatorname{SSS}(X)$ is bounded by the number of atoms. In the case of the braid group $B_{n}$ with the usual Garside structure, the number of atoms is $n-1$, hence one just needs to perform $n-1$ conjugations instead of $n$ !, for each element in $\operatorname{SSS}(X)$. Moreover, the minimal simple elements for a given $Y \in \operatorname{SSS}(X)$ can be computed very fast [22].

Notice that the algorithm just described computes not only the set $\operatorname{SSS}(X)$, but also the minimal simple elements that connect the elements in $\operatorname{SSS}(X)$ by conjugations. In other words, the algorithm computes a directed graph whose vertices are the elements in $\operatorname{SSS}(X)$, and whose arrows are defined as follows: there is an arrow labeled by $\rho$ starting at $Y$ and ending at $Z$ if $\rho$ is a minimal simple element for $Y$ and $Y^{\rho}=Z$. In Figure 3 one can see the graph associated to $\sigma_{1} \in B_{4}$. Notice that there are exactly 3 arrows starting at every vertex (the number of atoms in $B_{4}$ ). In general, the number of arrows starting at a given vertex can be smaller or equal, but never bigger than the number of atoms.

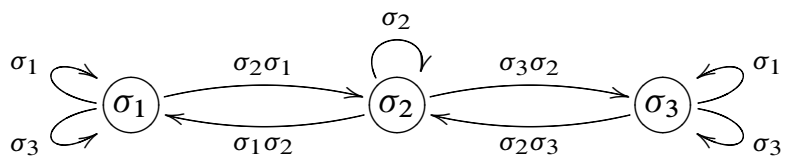

Figure 3. Graph associated to $\operatorname{SSS}\left(\sigma_{1}\right)$ in $B_{4}$. 
Let us mention here a tool that will be used several times in this paper, which is the transport map introduced in [24]. Let $X \in \operatorname{SSS}(X)$ and let $\alpha$ be an element such that $\alpha^{-1} X \alpha=Y \in \operatorname{SSS}(X)$. We can write this as $X \stackrel{\alpha}{\longrightarrow} Y$. We know from [20] that $\mathbf{c}(X)$ and $\mathbf{c}(Y)$ also belong to $\operatorname{SSS}(X)$. Notice that $X \stackrel{\iota(X)}{\longrightarrow} \mathbf{c}(X)$ and $Y \stackrel{\iota(Y)}{\longrightarrow} \mathbf{c}(Y)$. In [24], the transport $\alpha^{(1)}$ of $\alpha$ is defined as the element making the following diagram commutative in the sense explained below:

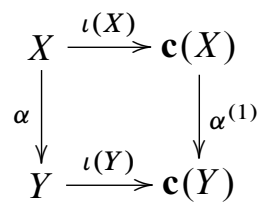

This means $\alpha^{(1)}=\iota(X)^{-1} \alpha \iota(Y)$. The nontrivial fact shown in [24] is that if $\alpha$ is simple, then $\alpha^{(1)}$ is simple.

At this point, the size of the set of simple elements is no longer a problem for the complexity of the algorithm, but there is still a big problem to handle: The size of $\operatorname{SSS}(X)$ is, in general, very big. The most recent improvement, given in [24], is to define a small subset of $\operatorname{SSS}(X)$ satisfying all the good properties described above, so that a similar algorithm can be used to compute it. The definition of this new subset appeared after observing that the cycling function maps $\operatorname{SSS}(X)$ to itself. As $\operatorname{SSS}(X)$ is finite, iterated cycling of any representative of $\operatorname{SSS}(X)$ must eventually become periodic. Hence it is natural to define the following:

Definition 1.15. Given $X \in G$, define the ultra summit set of $X$, $\operatorname{USS}(X)$, to be the set of elements $Y \in \operatorname{SSS}(X)$ such that $\mathbf{c}^{m}(Y)=Y$, for some $m>0$.

The ultra summit set USS $(X)$ thus consists of a (finite) set of disjoint, closed orbits under cycling. For instance, in the braid group $B_{n}$ one has $\operatorname{USS}\left(\sigma_{1}\right)=\operatorname{SSS}\left(\sigma_{1}\right)=$ $S S\left(\sigma_{1}\right)=\left\{\sigma_{1}, \ldots, \sigma_{n-1}\right\}$, and each element corresponds to an orbit under cycling, since $\mathbf{c}\left(\sigma_{i}\right)=\sigma_{i}$ for $i=1, \ldots, n-1$. A less trivial example is given by the element

$$
X=\sigma_{1} \sigma_{3} \sigma_{2} \sigma_{1} \cdot \sigma_{1} \sigma_{2} \cdot \sigma_{2} \sigma_{1} \sigma_{3} \in B_{4} .
$$

In this example $\operatorname{USS}(X)$ has 6 elements, while $\operatorname{SSS}(X)$ has 22 elements. More precisely, the ultra summit set of $X$ consists of 2 closed orbits under cycling, $\operatorname{USS}(X)=$ $\mathrm{O}_{1} \cup \mathrm{O}_{2}$, each one containing 3 elements:

$$
\begin{gathered}
O_{1}=\left\{\sigma_{1} \sigma_{3} \sigma_{2} \sigma_{1} \cdot \sigma_{1} \sigma_{2} \cdot \sigma_{2} \sigma_{1} \sigma_{3}, \sigma_{1} \sigma_{2} \cdot \sigma_{2} \sigma_{1} \sigma_{3} \cdot \sigma_{1} \sigma_{3} \sigma_{2} \sigma_{1},\right. \\
\left.\sigma_{2} \sigma_{1} \sigma_{3} \cdot \sigma_{1} \sigma_{3} \sigma_{2} \sigma_{1} \cdot \sigma_{1} \sigma_{2}\right\}, \\
O_{2}=\left\{\sigma_{3} \sigma_{1} \sigma_{2} \sigma_{3} \cdot \sigma_{3} \sigma_{2} \cdot \sigma_{2} \sigma_{3} \sigma_{1}, \sigma_{3} \sigma_{2} \cdot \sigma_{2} \sigma_{3} \sigma_{1} \cdot \sigma_{3} \sigma_{1} \sigma_{2} \sigma_{3},\right. \\
\left.\sigma_{2} \sigma_{3} \sigma_{1} \cdot \sigma_{3} \sigma_{1} \sigma_{2} \sigma_{3} \cdot \sigma_{3} \sigma_{2}\right\} .
\end{gathered}
$$


Notice that $O_{2}=\tau\left(O_{1}\right)$. Notice also that the cycling of every element in $\operatorname{USS}(X)$ gives another element which is already in left normal form, hence iterated cyclings correspond to cyclic permutations of the factors in the left normal form. We will say that elements satisfying this property are rigid. The precise definition will be given in $\S 1.4$. We remark that the size of the ultra summit set of a generic braid of canonical length $l$ is either $l$ or $2 l$ [24]. This means that, in the generic case, ultra summit sets consist of one or two orbits (depending on whether $\tau\left(O_{1}\right)=O_{1}$ or not), containing rigid braids.

The algorithm given in [24] to solve the CDP/CSP in Garside groups (of finite type) is analogous to the previous ones, but this time one needs to compute $\operatorname{USS}(X)$ instead of SSS $(X)$. In order to do this, the following results, which are analogous to those given for super summit sets, are used.

Theorem 1.16 ([24]). Let $X \in \operatorname{USS}(X)$. If $s, t \in G$ are such that $X^{s} \in \operatorname{USS}(X)$ and $X^{t} \in \operatorname{USS}(X)$, then $X^{s \wedge t} \in \operatorname{USS}(X)$.

Corollary 1.17 ([24]). Let $X \in G$ and $Y \in \operatorname{USS}(X)$. For every $u \in P$ there is a unique $\preccurlyeq$-minimal element $c_{Y}(u)$ satisfying

$$
u \preccurlyeq c_{Y}(u) \text { and } Y^{c_{Y}(u)} \in \operatorname{USS}(X) .
$$

Definition 1.18. Given $X \in G$ and $Y \in \operatorname{USS}(X)$, we say that a simple element $s \neq 1$ is a minimal simple element for $Y$ with respect to $\operatorname{USS}(X)$ if $Y^{s}=s^{-1} Y s \in \operatorname{USS}(X)$, and no proper prefix of $s$ satisfies this property.

Notice that the set of minimal simple elements for $Y$ with respect to $\operatorname{USS}(X)$ is contained in $c_{Y}(A)=\left\{c_{Y}(a) ; a\right.$ is an atom $\}$, hence the number of minimal simple elements for $Y$ is bounded by the number of atoms. For the rest of the paper, all minimal simple elements will be considered with respect to ultra summit sets (and not super summit sets).

Corollary 1.19 ([24]). Let $X \in G$ and $\mathcal{V} \subset \operatorname{USS}(X)$ be non-empty. If $\mathcal{V} \neq \operatorname{USS}(X)$ then there exist $Y \in \mathcal{V}$ and an atom a such that $c_{Y}(a)$ is a minimal simple element for $Y$, and $Y^{c_{Y}(a)} \in \operatorname{USS}(X) \backslash \mathcal{V}$.

In [24] it is shown how to compute the minimal simple elements corresponding to a given $Y \in \operatorname{USS}(X)$, hence one can compute the whole $\operatorname{USS}(X)$ starting by a single element $\tilde{X} \in \operatorname{USS}(X)$.

As above, the algorithm in [24] not only computes USS $(X)$ but also a graph which determines the conjugating elements. This graph is defined as follows.

Definition 1.20. Given $X \in G$, the directed graph $\Gamma_{X}$ is defined by the following data: 
(1) The set of vertices is $\operatorname{USS}(X)$.

(2) For every $Y \in \operatorname{USS}(X)$ and every minimal simple element $s$ for $Y$ with respect to $\operatorname{USS}(X)$, there is an arrow labeled by $s$ going from $Y$ to $Y^{s}$.

We remark that one obtains an element $\tilde{X} \in \operatorname{USS}(X)$ by iterated application of cycling to an element in $\operatorname{SSS}(X)$, which we know how to compute using cyclings and decyclings. The number of times one needs to apply cycling, in order to go from an element in $\operatorname{SSS}(X)$ to an element in $\operatorname{USS}(X)$ is not known in general. Nevertheless, the theoretical complexity of the algorithm in [24] is not worse than the one of the algorithm in [22], and is substantially better in practice, at least for braid groups.

In fact, it follows from the work in [23], [20], [12], [22], [24] discussed above, that the complexity of CDP/CSP for two elements $X, Y$ in a Garside group ( $\|X\| \geq\|Y\|)$ is $O(|\operatorname{USS}(X)| p+q)$, where $p$ is a polynomial in $\|X\|$ and the number of atoms, and $q$ is related to the number of times one must apply cycling to an element in $\operatorname{SSS}(X)$ to transform it into an element in $\operatorname{USS}(X)$. We believe that the second term $q$ is negligible compared to $|\operatorname{USS}(X)| p$, so our main interest is in trying to bound the size of the ultra summit set of an element in a Garside group.

In the particular case of braid groups, the size and structure of an ultra summit set happen to depend heavily on the geometrical properties of the braid, more precisely, on its Nielsen-Thurston type. This is explained next.

1.3. The Thurston-Nielsen trichotomy in the braid groups. The braid group $B_{n}$ is isomorphic to the mapping class group $\pi_{0}\left(\operatorname{Diff}_{+}\left(D_{n}^{2}\right)\right)$ of the disc with $n$ points removed. Admissible diffeomorphisms preserve orientation, fix $\partial D^{2}$ pointwise and fix the $n$ punctures or distinguished points setwise. Admissible isotopies fix both pointwise. As a mapping class group, $B_{n}$ has structure which, at this time, has not been fully related to its Garside structure, although some interesting relation between the two structures can be found in [19]. We will use the geometric structure in $\$ 3.4$ and also in [9], [10], so we describe what we need here. The structure that we describe had its origins in three very long papers of J. Nielsen [35], written in the 1930s, but the grand sweep of the theory was not recognized until much later, in the work of W. Thurston [38]. We refer to it as the Thurston-Nielsen trichotomy. There are many ways to describe it. We choose one which is based upon the action of $B_{n}$ on isotopy classes of simple closed curves (scc) on $D_{n}^{2}$. The scc considered in $D_{n}^{2}$ are nondegenerate, which means that they bound neither a single puncture nor all punctures (otherwise they could be collapsed to a puncture or isotoped to the boundary).

Theorem 1.21 ([38], [35]). Let $X \in B_{n}$. Then, after a suitable isotopy, $X$ belongs to exactly one of the following pairwise disjoint classes:

(1) $X$ is 'periodic'. That is, some power of $X$ is a power of a Dehn twist on $\partial D_{n}^{2}$ (this Dehn twist is precisely $\Delta^{2}$, with the usual Garside structure). 
(2) $X$ is 'pseudo-Anosov' or PA. That is, neither $X$ nor any power of $X$ fixes the isotopy class of any scc on $D_{n}^{2}$. This case is the generic case.

(3) $X$ is 'reducible'. That is, $X$ is not periodic and there exists a family of scc on $D_{n}^{2}$ whose isotopy class is fixed by $X$, so that some power $X^{m}$ of $X$ fixes the isotopy class of each simple closed curve in the family. Moreover, if the disc $D_{n}^{2}$ is split open along suitable representatives of the fixed curves, then the restriction of $X^{m}$ to the closure of each component of the split-open disc is either periodic or PA.

We note that there is a working algorithm, given in [4], [5], to determine whether a given braid is periodic or reducible, and we used it in basic ways when we computed the millions of examples that suggested the different structures of ultra summit sets, depending on the geometric type. Note that if one can recognize whether a braid is periodic or reducible, then if it is neither it must be PA.

\subsection{A project to solve the conjugacy problems in braid groups and a summary} of our results. Making use of almost all of the ideas that we have just described, we have developed a strategy for attacking the problem of the complexity of the conjugacy decision and search problems (CDP/CSP) in the braid groups. It uses the structure of centralizers of PA braids [26], and the uniqueness of their roots [25], some particular properties of periodic braids, and also the geometric decomposition of a reducible braid along its invariant curves. Hence, our strategy does not apply to an arbitrary Garside group, although many of the results that we show (all results in [9] and all results in this paper, except Theorem 3.37 and those in \$3.4) are stated and hold in the general framework of Garside groups. The results in [10] and the remaining parts of our project are conceived for braid groups, although we believe that they will probably be generalized to other Garside groups, at least to spherical type Artin-Tits groups.

As was noted in the previous sections, two elements $X, Y$ in a Garside group are conjugate if and only if one element in $\operatorname{USS}(X)$ is also in $\operatorname{USS}(Y)$. This means that we must compute all of $\operatorname{USS}(Y)$ in order to be able to test conjugacy. Thus we will need to understand the structure and size of the ultra summit set. Unfortunately, however, $\mathrm{USS}(X)$ can be quite complicated, partly because cycling is not, in general, a cyclic permutation of the factors in a left normal form, but also because it is not clear how the distinct orbits in $\operatorname{USS}(X)$ are related. The former problem is avoided if $\operatorname{USS}(X)$ is made of rigid elements.

In Section 3 we will introduce and study rigid elements. Let $X=\Delta^{p} x_{1} x_{2} \ldots x_{r}$ be in left normal form. Assume that $r>0$. Then $X$ is rigid if $\Delta^{p} x_{1} x_{2} \ldots x_{r} \tau^{-p}\left(x_{1}\right)$ is in normal form as written. We were lead to study rigid elements when we realized, long ago, that it was often very difficult to predict and understand the changes in normal form of braids after cycling. If $X$ is rigid, the left normal form of $\mathbf{c}(X)$ is precisely $\Delta^{p} x_{2} \ldots x_{r} \tau^{-p}\left(x_{1}\right)$, so cycling is simpler than in the general case, and the 
combinatorics in USS $(X)$ are easier to understand.

In this paper we will see that obtaining a polynomial solution to the CDP/CSP for certain elements in a Garside group, reduces to obtaining such a solution for rigid elements. In the case of braid groups, this happens for pseudo-Anosov (PA) braids. Since the property of being PA is generic in $B_{n}$, this is an important step in the case of braids.

Assuming that $X, Y \in B_{n}$, we consider the three cases separately: $X, Y$ are PA, periodic or reducible. We break our approach to $B_{n}$ into the following six steps:

I Determining if a braid is periodic, reducible or PA. We remark that it is very fast to decide whether a given braid is periodic [25], so the main problem is to determine if a braid is reducible, and find the reducing curves. This question is solved in [4], [5], but the proposed algorithm computes $\operatorname{SSS}(X)$. In fact, one can replace $\operatorname{SSS}(X)$ by $\operatorname{USS}(X)$, but having to compute $\operatorname{USS}(X)$ means that the algorithm is not polynomial, in general (in [10] there are examples of USS's in $B_{n}$ whose size is exponential in $n$ ). This yields the following.

Open question 1: Is there an algorithm to determine if a braid in $B_{n}$ is reducible and to find its reducing curves, which is polynomial in $n$ and $\|X\|$ ?

This problem was first studied in [4]. Some work in this direction can be found in [29]. There is also an algorithm in [8] which solves the above problem, but it is not polynomial, to our knowledge.

II PA braids: passing to powers. In Sections 2 and 3 of this paper we show that if $X$ and $Y$ are PA, there is some small power $m$ such that $\operatorname{USS}\left(X^{m}\right)$ is made of rigid braids, and it suffices to solve the CDP/CSP for $X^{m}$ and $Y^{m}$.

In this regard we make two remarks: The first is that, in view of the results in [25], for every nonzero integer $m$, the braids $X$ and $Y$ are conjugate if and only if $X^{m}$ and $Y^{m}$ are conjugate. Furthermore, $P A$ braids have unique roots. Hence if $X$ and $Y$ are $P A$, and $Z$ conjugates $X^{m}$ to $Y^{m}$, then $Z$ conjugates $X$ to $Y$. Therefore nothing is lost in passing to powers.

Our second remark is that we prove the non-emptiness of the stable ultra summit set $\mathrm{SU}(X)$ in a Garside group (compare with [30], where the stable super summit set is introduced). That is, for every $X \in G$ define $\operatorname{SU}(X)=\{Y \in$ $\mathrm{USS}(X) ; Y^{k} \in \mathrm{USS}\left(Y^{k}\right)$ for all $\left.k \in \mathbb{Z}\right\}$. Proposition 2.23 of this paper proves that $\mathrm{SU}(X) \neq \varnothing$. However, we will not need to work in $\mathrm{SU}(X)$, it will suffice to control a bounded number of powers of $X$, and we learn how to do that.

III Understanding the USS graph. In [9] we uncover and study the structure of the ultra summit set of an element in a Garside group. More precisely, we show that the conjugations corresponding to minimal simple elements (the arrows in the USS graph) are a very special kind of conjugation that we call partial cyclings. This work is not restricted to braids. At the end of [9] we specialize our work to 
the cases: (a) $X$ is a rigid element, and (b) $X$ is a periodic element (in a Garside group, that is, a root of some power of $\Delta$ ). This is a first step towards the solution of the following.

IV Finding a polynomial bound for the size of $\operatorname{USS}(X)$, when $X$ is rigid. At this writing this work is incomplete. We have computed many many examples, using random searches, and on the basis of the evidence found that in the generic case $\operatorname{USS}(X)$ has either 2 orbits, where one is the conjugate of the other by $\Delta$, or 1 orbit which is conjugate to itself by $\Delta$. However, there are exceptional cases where $\operatorname{USS}(X)$ has unexpected size. There is no indication whatsoever of uncontrolled growth. Indeed, the combinatorial conditions that are uncovered in [9] are so restrictive that exponential growth seems very unlikely. But since we do not have an affirmative answer, we state the following.

Open question 2: If $X$ is a rigid element in $B_{n}$, is the size of $\operatorname{USS}(X)$ bounded above by some polynomial in $n$ and $\|X\|$ ?

We remark that, in $\$ 3.2$ of this paper, we show that if $X \in G$ is a rigid element of canonical length greater than 1, then $\operatorname{USS}(X)$ consists of rigid elements.

Finally, solving Open question 2 affirmatively would imply that the algorithm in [24] applied to rigid braids is polynomial in $n$ and $\|X\|$, provided that the following is also true, at least for conjugates of a rigid braid:

Open question 3: Given $X \in B_{n}$ and $Y \in \operatorname{SSS}(X)$, let $m$ be such that $\mathbf{c}^{m}(Y) \in$ $\mathrm{USS}(X)$. Is $m$ bounded above by a polynomial in $n$ and $\|X\|$ ?

V Periodic braids. In [10] we settle the CSP for periodic braids in $B_{n}$, in polynomial time with respect to $n$ and $\|X\|$. We remark that the CDP for periodic braids was already known to be polynomial [4], [5], but the usual algorithm to solve the CSP is not polynomial in this case, so in [10] we find a new specific algorithm for periodic braids.

VI Reducible braids. Suppose that Open question 1, 2 and 3 above are solved. Note that reducible braids are braids that are made up of braided tubes, each containing braided tubes and so forth until one reaches an irreducible braid, which is then either periodic or PA. Once that reducing curves are known, and one knows how to solve the CDP/CSP for irreducible braids, one can use techniques from [25], namely Proposition 3.2, in the following way. First one solves the CSP for the external (or tubular) braids, so one can assume that the two braids one started with, $X$ and $Y$, have the same associated tubular braid, say $\hat{X}$. Then one needs to solve the CDP/CSP for the interior braids (the braids inside the tubes). Proposition 3.2 in [25] states that $X$ and $Y$ will be conjugate if and only if each interior braid of $X$ is conjugate to an interior braid of $Y$, and there is an element in the centralizer of $\hat{X}$ whose permutation sends each interior braid of $X$ to the tube in which one finds its corresponding conjugate in $Y$. Notice that the tubular 
braid is no longer reducible, hence it is either periodic or pseudo-Anosov. If it is periodic, then its centralizer is well known [7] and easy to compute, with the help of the algorithm in [10]. If it is pseudo-Anosov, it is known that its centralizer is isomorphic to $\mathbb{Z}^{2}$, but we don't know an algorithm to compute the two generators it in polynomial time. In any case, if $X$ is pseudo-Anosov, the centralizer of $X$ equals the centralizer of $X^{m}$ for every nonzero $m$, since pseudo-Anosov elements have unique roots [25]. Therefore, using the results in this paper, one can assume that $X$ is rigid, and the only thing that remains to be shown, in the reducible case, is the following.

Open question 4: Given $X \in B_{n}$ a pseudo-Anosov, rigid braid, is there an algorithm polynomial in $n$ and $\|X\|$ that computes two free generators of the centralizer of $X$ ?

As a conclusion, the work in this paper and in [9], [10], together with an affirmative answer to Open questions 1, 2, 3 and 4 above, would yield a polynomial algorithm to solve the CDP/CSP in braid groups. Due to our increasing understanding of the structure of ultra summit sets, we believe that this final goal is within reach.

In this paper we will solve problem II above. Most of our results (except those in $\$ 3.4$ and Theorem 3.37) hold in all Garside groups.

In $\S 2$ we determine the relationship between the $m$-times iterated cycling $\mathbf{c}^{m}(X)$ of $X \in G$ and the $m^{\text {th }}$ power $X^{m}$ of $X$. The main result is Theorem 2.9 of $\S 2.2$. In $\$ 2.4$ we introduce the stable ultra summit set $\mathrm{SU}(X)$ of $X \in G$ (cf [30]) and give a short proof that it is non-empty. While we realized, after we had completed the work in this paper, that we did not really need $\mathrm{SU}(X)$ in our work, we include it for completeness, and because it may be useful for others.

In $\S 3$ we study rigid elements in Garside groups and prove some surprising results about them. In particular, in Theorem 3.15 we prove that if $X$ is rigid and $\ell(X)>1$ then every element in USS $(X)$ is also rigid. Theorem 3.21 characterizes exactly which elements in a Garside group have rigid powers. Using it, we prove in Theorem 3.23 that if $X$ is a pseudo-Anosov braid in its USS, then there exists an $m$ such that $X^{m}$ is rigid. In Theorem 3.34 we solve the problem that is described in II above in this section, obtaining a polynomial bound for the power $m$.

Remark. As this paper was about to go to press, the second and third authors discovered a way to avoid solving open problem 1, provided open problems 2, 3 and 4 are solved. A manuscript does not yet exist, so that, unfortunately, we cannot give a reference. 


\section{Cyclings and powers}

Recall the definition of a rigid element in a Garside group $G$ (Definition 3.1). Our goal in this paper is to understand the conditions under which an element $X \in \operatorname{USS}(X) \subset$ $G$ which is not necessarily rigid has a small power $X^{m}$ which is rigid. This will be done by investigating the relationship between iterated cyclings and iterated powers of $X$. However, the connection between cycling and normal forms of powers is fairly subtle. The problems that we will encounter and solve will be easier to understand after we study an example. They will probably have been encountered by others who have worked with left normal forms (see [1], [20], [21]) in the braid group $B_{n}$, and struggled to understand how they change after cycling.

An example. Let $X=\Delta^{p} x_{1} \ldots x_{r} \in \operatorname{USS}(X) \subset G, r>0$. Since $\inf (X)=p$, $\ell(X)=r$, it is immediate that $\inf \left(X^{m}\right) \geq m p$ and that $\ell\left(X^{m}\right) \leq m r$. We can think of the terms $\Delta^{m p}$ in the normal form of $X^{m}$ as the expected $\Delta^{\prime} s$, and any additional ones as unexpected $\Delta^{\prime} s$. Similarly, $\sup (X)=p+r$, so that $m p+m r$ is the expected supremum of $X^{m}$. There will be an unexpected decrease in $\sup \left(X^{m}\right)$ if and only if the actual value of $\sup \left(X^{m}\right)$ is less than $m p+m r$. These two issues are closely related, because by Corollary $1.6 \sup (X)=-\inf \left(X^{-1}\right)$ and $\inf (X)=-\sup \left(X^{-1}\right)$, so that if we arrive at an understanding of unexpected increases in the infimum, we will also have arrived at an understanding of unexpected decreases in the supremum. Unfortunately, however, the normal form of $X^{m}$ is not easily related to the normal form of $X$, as is illustrated by the following example, taken from the 5-string braid group $B_{5}$.

Let $X=12132143143 \in B_{n}$, where the letter $i$ means the elementary braid $\sigma_{i}$. In this example $\inf (X)=0$. A calculation shows that $X$ is in its ultra summit set, and there are 2 cycling orbits in USS $(X)$, each with 4 elements, with the second being the conjugate of the first by $\Delta$. In this simple case $\ell(X)=2$, that is, there are 2 simple factors in the left normal form for $X=C_{1} \cdot R_{1}$, where dots are used to separate the simple words in the left normal form. Here is the first orbit:

$$
\begin{aligned}
X & =12132143 \cdot 143=C_{1} \cdot R_{1}, \\
\mathbf{c}(X) & =121324321 \cdot 14=C_{2} \cdot R_{2}, \\
\mathbf{c}^{2}(X) & =12132432 \cdot 214=C_{3} \cdot R_{3}, \\
\mathbf{c}^{3}(X) & =121343 \cdot 12324=C_{4} \cdot R_{4}, \\
\mathbf{c}^{4}(X) & =X .
\end{aligned}
$$

What about powers of $X$ ? Calculating left normal forms, we find that:

$$
\begin{aligned}
X & =12132143 \cdot 143, \\
X^{2} & =\Delta \cdot 2324321 \cdot 14 \cdot 143,
\end{aligned}
$$




$$
\begin{aligned}
& X^{3}=\Delta^{2} \cdot 12324 \cdot 214 \cdot 14 \cdot 143 \\
& X^{4}=\Delta^{2} \cdot 12132143 \cdot 143 \cdot 12324 \cdot 214 \cdot 14 \cdot 143 \\
& X^{5}=\Delta^{3} \cdot 2324321 \cdot 14 \cdot 143 \cdot 12324 \cdot 214 \cdot 14 \cdot 143
\end{aligned}
$$

Since $\inf (X)=0$, the powers $X^{2}, X^{3}, X^{4}, X^{5}$ have 1, 2, 2, 3 unexpected $\Delta^{\prime} s$.

A hint at how the normal forms of $X$ and $X^{m}$ might be related comes from a more careful inspection of this example (and many many other examples like it). The initial factors of the elements in the orbit of $X$ are $C_{1}=12132143, C_{2}=121324321$, $C_{3}=12132432, C_{4}=121343$. These are the 'conjugating factors' that are used when we cycle, that is, if $\boldsymbol{C}_{m}=C_{1} C_{2} \ldots C_{m}$, then $\mathbf{c}^{m}(X)=X^{\boldsymbol{C}_{m}}$. Here are the left normal forms for $\boldsymbol{C}_{1}, \boldsymbol{C}_{2}, \ldots, \boldsymbol{C}_{5}$ :

$$
\begin{aligned}
\boldsymbol{C}_{1} & =12132143=\text { first simple factor in } X, \\
\boldsymbol{C}_{2} & =12132143121324321 \\
& =\Delta \cdot 2324321 \\
& =\text { product of first } 2 \text { simple factors in } X^{2}, \\
\boldsymbol{C}_{3} & =1213214312132432112132432 \\
& =\Delta^{2} \cdot 12324 \\
& =\text { product of first } 3 \text { simple factors in } X^{3}, \\
\boldsymbol{C}_{4} & =\Delta^{2} \cdot 12132143 \cdot 143=\text { product of first } 4 \text { simple factors in } X^{4}, \\
C_{5} & =\Delta^{3} \cdot 2324321 \cdot 14=\text { product of first } 5 \text { simple factors in } X^{5} .
\end{aligned}
$$

One of the main results in this paper states that, for every $X \in \operatorname{USS}(X)$, the product of the first $m$ factors in the left normal form of $X^{m} \Delta^{-m p}$, where we include powers of $\Delta$ in the count, is precisely $\boldsymbol{C}_{m}$, the product of the conjugating elements involved in the first $m$ cyclings of $X$. This will allow us to determine which elements admit a rigid power and, under some hypothesis, we find an upper bound for the smallest power which is rigid. In the particular case of braid groups, these results apply to pseudo-Anosov braids, since we will show in $\$ 3.4$ that every pseudo-Anosov braid in its ultra summit set has a rigid power.

2.1. Decomposition of powers of $\boldsymbol{X}$. In this section we will decompose $X^{m}$ as a product of two elements, each of which is determined by the iterated cyclings of $X \in G$. Assume from now on that $X \in \operatorname{SSS}(X)$ and $\ell(X) \geq 1$. We will develop some basic properties of this decomposition for elements of a Garside group.

We first need some notation. If the left normal form of $X$ is $\Delta^{p} x_{1} \ldots x_{r}$, recall that $\iota(X)=\tau^{-p}\left(x_{1}\right)$ is the initial factor of $X$, and that the cycling of $X$ is defined by $\mathbf{c}(X)=X^{\iota(X)}$. If we apply iterated cyclings to $X$, the conjugating elements will be denoted by $C_{1}, C_{2}, \ldots$ That is, $C_{i}=\iota\left(\mathbf{c}^{i-1}(X)\right)$ for $i \geq 1$. Hence one has 
$\mathbf{c}^{m}(X)=X^{C_{1} \ldots C_{m}}$. The letter $C$ in the symbol $C_{i}$ comes from conjugating element, since one conjugates $\mathbf{c}^{i-1}(X)$ by $C_{i}$ to obtain $\mathbf{c}^{i}(X)$.

The element $X$ can be decomposed as follows: $X=\Delta^{p} x_{1} \ldots x_{r}$ $=C_{1} \Delta^{p} x_{2} \ldots x_{r}$. We denote $R_{1}=x_{2} \ldots x_{r}$, so $X=C_{1} \Delta^{p} R_{1}$. For the iterated cyclings of $X$, we denote $R_{i}$ in a similar way, that is, the element satisfying $\mathrm{c}^{i-1}(X)=C_{i} \Delta^{p} R_{i}$. The letter $R$ comes from remainder. Notice that every $C_{i}$ is a simple element, while $R_{i}$ is simple only if $\ell(X) \leq 2$, and it is trivial if $\ell(X)=1$. The important fact about these elements relies on how they behave when they are multiplied in the right way.

Definition 2.1. Let $X \in \operatorname{SSS}(X)$ with $\inf (X)=p$ and $\ell(X) \geq 1$. For $i \geq 1$, let $C_{i}$ and $R_{i}$ be the elements defined above. Then, for every $m \geq 1$, we define:

- $C_{m}=C_{1} \ldots C_{m}$.

- $\boldsymbol{R}_{m}=\tau^{-p}\left(R_{m}\right) \tau^{-2 p}\left(R_{m-1}\right) \ldots \tau^{-m p}\left(R_{1}\right)$.

Notice that

$$
\boldsymbol{R}_{m} \Delta^{p m}=\left(\Delta^{p} R_{m}\right)\left(\Delta^{p} R_{m-1}\right) \ldots\left(\Delta^{p} R_{1}\right) .
$$

Since later we will deal not only with $X$, but with successive cyclings of $X$, we want to define the corresponding elements above, for $\mathbf{c}^{k}(X)$. Hence we define $\boldsymbol{C}_{[k, m]}$ and $\boldsymbol{R}_{[k, m]}$ to be the elements $\boldsymbol{C}_{m}$ and $\boldsymbol{R}_{m}$ above, but defined with respect to $\mathbf{c}^{k}(X)$. This yields the analogous definition with the indices shifted by $k$ :

Definition 2.2. Let $X \in \operatorname{SSS}(X)$ with $\inf (X)=p$ and $\ell(X) \geq 1$. For $i \geq 1$, let $C_{i}$ and $R_{i}$ be the elements defined above. Then, for every $m \geq 1$ and $k \geq 0$, we define:

- $C_{[k, m]}=C_{k+1} \ldots C_{k+m}$.

- $\boldsymbol{R}_{[k, m]}=\tau^{-p}\left(R_{k+m}\right) \tau^{-2 p}\left(R_{k+m-1}\right) \ldots \tau^{-m p}\left(R_{k+1}\right)$.

Notice that

$$
\boldsymbol{R}_{[k, m]} \Delta^{p m}=\left(\Delta^{p} R_{k+m}\right)\left(\Delta^{p} R_{k+m-1}\right) \ldots\left(\Delta^{p} R_{k+1}\right) .
$$

Clearly, $\boldsymbol{C}_{m}=\boldsymbol{C}_{[0, m]}$ and $\boldsymbol{R}_{m}=\boldsymbol{R}_{[0, m]}$.

In the particular case in which $X \in \operatorname{USS}(X)$, that is, $X \in \operatorname{SSS}(X)$ and $\mathbf{c}^{t}(X)=X$ for some positive integer $t$, we can extend the above definition to negative values of $k$, as follows. We know that $\mathbf{c}^{m}(X) \in \operatorname{USS}(X)$ for every $m \geq 0$. If we denote by $\mathcal{O}(X)$ the orbit of $X$ under cycling, we can define $\mathbf{c}^{-m}(X)$ to be the element $Y \in \mathcal{O}(X)$ such that $\mathbf{c}^{m}(Y)=X$ (although cycling is not injective in the whole $G$, it is a bijection in $\operatorname{USS}(X)$, so we hope this notation will not cause confusion).

Recall that we defined $C_{i}=\iota\left(\mathrm{c}^{i-1}(X)\right)$, and $R_{i}$ in such a way that $\mathrm{c}^{i-1}(X)=$ $C_{i} \Delta^{p} R_{i}$, for every $i \geq 1$. The same definitions can now be given for every $i \in \mathbb{Z}$, as we have definitions for the negative cyclings of $X$. Since $\mathcal{O}(X)$ is a finite set, the 
sequences $\left\{C_{i}\right\}_{i \in \mathbb{Z}}$ and $\left\{R_{i}\right\}_{i \in \mathbb{Z}}$ are periodic. Therefore, we have definitions for the elements $C_{i}, R_{i}$, and also $\boldsymbol{C}_{[k, m]}$ and $\boldsymbol{R}_{[k, m]}$ for every $i, k \in \mathbb{Z}$ and every $m \geq 1$.

Let us show a result that will be useful later.

Lemma 2.3. Let $X \in \operatorname{SSS}(X)$ with $\inf (X)=p$ and $\ell(X) \geq 1$. For every $m, k \geq 1$ one has

$$
\boldsymbol{R}_{[k-1, m]} \Delta^{p m} C_{k}=C_{k+m} \boldsymbol{R}_{[k, m]} \Delta^{p m} .
$$

In other words,

$$
\left(\Delta^{p} R_{k+m-1}\right) \ldots\left(\Delta^{p} R_{k}\right) C_{k}=C_{k+m}\left(\Delta^{p} R_{k+m}\right) \ldots\left(\Delta^{p} R_{k+1}\right) .
$$

Moreover, if $X \in \operatorname{USS}(X)$, the same equality holds for every $k \in \mathbb{Z}$.

Proof. We first show the result for $X \in \mathrm{SSS}(X)$ and $k \geq 1$. If $m=1$, the result is true since $\left(\Delta^{p} R_{k}\right) C_{k}=\mathrm{c}^{k}(X)=C_{k+1}\left(\Delta^{p} R_{k+1}\right)$ by definition. Suppose the result true for $m-1$. Then one has

$$
\begin{aligned}
& \left(\Delta^{p} R_{k+m-1}\right)\left(\Delta^{p} R_{k+m-2}\right) \ldots\left(\Delta^{p} R_{k}\right) C_{k} \\
& \quad=\left(\Delta^{p} R_{k+m-1}\right) C_{k+m-1}\left(\Delta^{p} R_{k+m-1}\right) \ldots\left(\Delta^{p} R_{k+1}\right) \\
& \quad=\mathbf{c}^{k+m-1}(X)\left(\Delta^{p} R_{k+m-1}\right) \ldots\left(\Delta^{p} R_{k+1}\right) \\
& \quad=C_{k+m}\left(\Delta^{p} R_{k+m}\right)\left(\Delta^{p} R_{k+m-1}\right) \ldots\left(\Delta^{p} R_{k+1}\right)
\end{aligned}
$$

so the result is also true for $m$ and we are done.

If $X \in \operatorname{USS}(X)$ and $k \leq 0$, the same proof is valid.

We will now see how the element $X^{m}$ can be decomposed in terms of $\boldsymbol{C}_{m}$ and $\boldsymbol{R}_{m}$, together with some properties concerning the normal form of these two factors.

Lemma 2.4. Let $X \in \operatorname{SSS}(X)$, with $\ell(X) \geq 1$. Let $C_{i}, R_{i}, C_{m}$ and $\boldsymbol{R}_{m}$ be the elements defined above. Then:

(1) The $m$-th power of $X$ has the decomposition

$$
X^{m}=\boldsymbol{C}_{m} \boldsymbol{R}_{m} \Delta^{m p} .
$$

(2) In this decomposition, $\inf \left(\boldsymbol{R}_{m}\right)=0$ and $\iota\left(\boldsymbol{R}_{m}\right) \preccurlyeq C_{m+1}$, for every $m \geq 1$.

(3) In general $\inf \left(\boldsymbol{C}_{m}\right) \geq 0$. If $\ell(X)>1$, one has $\sup \left(\boldsymbol{C}_{m}\right)=m, \ell\left(\boldsymbol{C}_{m}\right)>0$ and $\varphi\left(\boldsymbol{C}_{m}\right) \succcurlyeq \varphi\left(\mathbf{c}^{m}(X)\right)$, for every $m \geq 1$.

Remark. The left normal form of $\boldsymbol{C}_{m}$ is not so easy to understand, as we saw in the example that was given at the beginning of Section 2. Uncovering it, and relating it to the left normal form of $X^{m}$, will be a major part of our investigations. 
Proof of Lemma 2.4. (1) For $m=1$ the result is clear, since $X=C_{1} \Delta^{p} R_{1}=$ $C_{1} R_{1} \Delta^{p}$ by definition. Now suppose that

$$
X^{m-1}=\boldsymbol{C}_{m-1} \boldsymbol{R}_{m-1} \Delta^{(m-1) p} .
$$

Then one has

$$
X^{m}=X^{m-1} X=C_{m-1} \boldsymbol{R}_{m-1} \Delta^{(m-1) p} C_{1}\left(\Delta^{p} R_{1}\right) .
$$

By Lemma 2.3 with $k=1$, it then follows that

$$
\begin{aligned}
X^{m} & =\boldsymbol{C}_{m-1} C_{m} \boldsymbol{R}_{[1, m-1]} \Delta^{(m-1) p}\left(\Delta^{p} R_{1}\right) \\
& =\boldsymbol{C}_{m} \boldsymbol{R}_{[1, m-1]} \Delta^{m p} R_{1} \\
& =\boldsymbol{C}_{m} \boldsymbol{R}_{[1, m-1]} \tau^{-m p}\left(R_{1}\right) \Delta^{m p} \\
& =\boldsymbol{C}_{m} \boldsymbol{R}_{m} \Delta^{m p},
\end{aligned}
$$

so the result is true for every $m \geq 1$.

(2) To prove that $\inf \left(\boldsymbol{R}_{m}\right)=0$ and that $\iota\left(\boldsymbol{R}_{m}\right) \preccurlyeq C_{m+1}$, for every $m \geq 1$, we notice that $\boldsymbol{R}_{m}$ is positive by definition. Hence both statements will follow if we can show that $\Delta \wedge \boldsymbol{R}_{m} \preccurlyeq C_{m+1}$.

If $m=1$ one has $\boldsymbol{R}_{1}=\tau^{-p}\left(x_{2} \ldots x_{r}\right)$, hence $\Delta \wedge \boldsymbol{R}_{1}=\tau^{-p}\left(x_{2}\right)$. We also have $C_{2}=\iota(\mathbf{c}(X))=\iota\left(\Delta^{p} x_{2} \ldots x_{r} \tau^{-p}\left(x_{1}\right)\right)$. Since $X \in \operatorname{SSS}(X)$, we have $\inf \left(x_{2} \ldots x_{r} \tau^{-p}\left(x_{1}\right)\right)=0$, so the first factor in its left normal form is equal to $x_{2} s$ for some simple element $s$. Hence $C_{2}=\iota\left(\Delta^{p} x_{2} \ldots x_{r} \tau^{-p}\left(x_{1}\right)\right)=\tau^{-p}\left(x_{2} s\right)$. Therefore $\Delta \wedge \boldsymbol{R}_{1}=\tau^{-p}\left(x_{2}\right) \preccurlyeq \tau^{-p}\left(x_{2} s\right)=C_{2}$, and the result is true for $m=1$.

Suppose that $\Delta \wedge \boldsymbol{R}_{m-1} \preccurlyeq C_{m}$ for some $m$. By definition, $\boldsymbol{R}_{m}=\tau^{-p}\left(R_{m} \boldsymbol{R}_{m-1}\right)$, hence

$$
\Delta \wedge \boldsymbol{R}_{m}=\Delta \wedge \tau^{-p}\left(R_{m} \boldsymbol{R}_{m-1}\right)=\tau^{-p}\left(\Delta \wedge\left(R_{m} \boldsymbol{R}_{m-1}\right)\right) .
$$

Notice that, since $\inf \left(\boldsymbol{R}_{m-1}\right)=0$ by the induction hypothesis, the initial factor of $R_{m} \boldsymbol{R}_{m-1}$ depends only on $R_{m}$ and on the initial factor of $\boldsymbol{R}_{m-1}$, that is,

$$
\Delta \wedge\left(R_{m} \boldsymbol{R}_{m-1}\right)=\Delta \wedge\left(R_{m}\left(\Delta \wedge \boldsymbol{R}_{m-1}\right)\right) \preccurlyeq \Delta \wedge\left(R_{m} C_{m}\right) .
$$

But $\Delta^{p} R_{m} C_{m}=\mathbf{c}^{m}(X)$, and $\iota\left(\mathbf{c}^{m}(X)\right)=C_{m+1}$, hence $\Delta \wedge\left(R_{m} C_{m}\right)=\tau^{p}\left(C_{m+1}\right)$. Therefore

$$
\Delta \wedge\left(R_{m} \boldsymbol{R}_{m-1}\right) \preccurlyeq \tau^{p}\left(C_{m+1}\right),
$$

and then

$$
\Delta \wedge \boldsymbol{R}_{m}=\tau^{-p}\left(\Delta \wedge\left(R_{m} \boldsymbol{R}_{m-1}\right)\right) \preccurlyeq \tau^{-p}\left(\tau^{p}\left(C_{m+1}\right)\right)=C_{m+1},
$$

as we wanted to show.

(3) We prove (3) by induction on $m$. If $m=1$ then $C_{1}=C_{1}$. As $\ell(X)>0, C_{1}$ is a non-trivial simple element, whence $\sup \left(C_{1}\right)=\ell\left(C_{1}\right)=1$. As $X \in \operatorname{SSS}(X)$, 
the number of canonical factors cannot decrease when passing from $X$ to $\mathbf{c}(X)$. In particular, the factor $C_{1}$ moved to the end cannot be absorbed completely which shows $\varphi\left(\boldsymbol{C}_{1}\right)=C_{1} \succcurlyeq \varphi(\mathbf{c}(X))$.

Suppose the result true for some $m \geq 1$ and let $F=\varphi\left(C_{m}\right)=\varphi\left(C_{1} \ldots C_{m}\right)$. As above we see that $\varphi\left(\mathbf{c}^{m}(X)\right) C_{m+1}$ cannot be simple, as $X$ (and hence $\mathbf{c}^{m}(X)$ ) is super summit. Notice that we used $\ell(X)>1$ here. Since $F \geqslant \varphi\left(\mathbf{c}^{m}(X)\right)$ by induction, this implies that $F C_{m+1}$ also has supremum 2. It is well known [34] that if one multiplies a left normal form $y_{1} \ldots y_{m}$ by a simple element $C_{m+1}$, then the left normal form of the product is computed by applying $m$ local transformations to pairs of consecutive factors, starting by the rightmost pair. In this way one can compute the normal form of every element, so we will refer to this as the normal form algorithm. Since $\sup \left(\boldsymbol{C}_{m}\right)=m$ by induction hypothesis, and $F C_{m+1}$ is not simple, the normal form algorithm implies that $\sup \left(\boldsymbol{C}_{m+1}\right)=\sup \left(C_{1} \ldots C_{m+1}\right)=m+1$. This algorithm together with $F \geqslant \varphi\left(\mathbf{c}^{m}(X)\right)$ and $\ell(X)>1$ also implies that

$$
\begin{aligned}
\varphi\left(\boldsymbol{C}_{m+1}\right) & =\varphi\left(C_{1} \ldots C_{m} C_{m+1}\right) \\
& =\varphi\left(F C_{m+1}\right) \succcurlyeq \varphi\left(\varphi\left(\mathbf{c}^{m}(X)\right) C_{m+1}\right)=\varphi\left(\mathbf{c}^{m+1}(X)\right) .
\end{aligned}
$$

Finally, since $C_{m+1} \neq \Delta$ and $F C_{m+1}$ has supremum 2, it follows that $\varphi\left(\boldsymbol{C}_{m+1}\right)=$ $\varphi\left(F C_{m+1}\right) \neq \Delta$, so $\ell\left(C_{m+1}\right)>0$.

Since the super summit set of an element is closed under cycling, Lemma 2.4 is still true if we apply it to every iterated cycling of $X$. If furthermore $X \in \operatorname{USS}(X)$, the same will be true for every element in $\mathcal{O}(X)$. This yields the following result.

Lemma 2.5. Let $X \in \mathrm{SSS}(X)$, with $\ell(X) \geq 1$. With the above notation, one has, for every $k \geq 0$ and every $m \geq 1$ :

(1) The $m$-th power of $\mathbf{c}^{k}(X)$ has the decomposition

$$
\left(\mathbf{c}^{k}(X)\right)^{m}=\boldsymbol{C}_{[k, m]} \boldsymbol{R}_{[k, m]} \Delta^{m p} .
$$

(2) In this decomposition, $\inf \left(\boldsymbol{R}_{[k, m]}\right)=0$ and $\iota\left(\boldsymbol{R}_{[k, m]}\right) \preccurlyeq C_{k+m+1}$, for every $m \geq 1$.

(3) In general inf $\left(\boldsymbol{C}_{[k, m]}\right) \geq 0$. If $\ell(X)>1$, one has $\sup \left(\boldsymbol{C}_{[k, m]}\right)=m$, $\ell\left(\boldsymbol{C}_{[k, m]}\right)>0$ and $\varphi\left(\boldsymbol{C}_{[k, m]}\right) \geqslant \varphi\left(\mathbf{c}^{k+m}(X)\right)$, for every $m \geq 1$.

Moreover, if $X \in \mathrm{USS}(X)$, the result holds for every $k \in \mathbb{Z}$.

Notice that in $\boldsymbol{C}_{[k, m]}$ and $\boldsymbol{R}_{[k, m]}$, the first index determines an element in the cycling orbit of $X$, and the second index determines its power. One can also think of $m$ as being the number of factors in the decompositions of $\boldsymbol{C}_{[k, m]}$ and $\boldsymbol{R}_{[k, m]}$ given by the definitions. But this is not necessarily the number of factors in their normal forms. 
2.2. Interplay between $\boldsymbol{C}_{\boldsymbol{m}}$ and $\boldsymbol{R}_{\boldsymbol{m}}$. Having proved that $X^{m}=\boldsymbol{C}_{m} \boldsymbol{R}_{m} \Delta^{p m}$, we will show that if $X$ belongs to its ultra summit set, this decomposition is left weighted, that is, $\varphi\left(\boldsymbol{C}_{m}\right) \iota\left(\boldsymbol{R}_{m}\right)$ is in left normal form as written. In other words, since we know by Lemma 2.4 that $\sup \left(\boldsymbol{C}_{m}\right)=m$, we will show that the product of the first $m$ factors, including $\Delta$ 's, in the left normal form of $X^{m} \Delta^{-p m}$ is precisely $\boldsymbol{C}_{m}$.

If $X \in \operatorname{USS}(X)$, recall that by Lemma 2.3 , one has

$$
\boldsymbol{R}_{[k-1, m]} \Delta^{p m} C_{k}=C_{k+m} \boldsymbol{R}_{[k, m]} \Delta^{p m},
$$

for every $k \in \mathbb{Z}$. We will actually see that the initial factor of this element is precisely $C_{k+m}$, no matter how many remainders we multiply on the right, that is, no matter how big is $m$.

Lemma 2.6. Let $X \in \operatorname{SSS}(X)$ with $\inf (X)=p$ and $\ell(X) \geq 1$. For every $m \geq 1$ and $k \geq 0$, one has

$$
\left(C_{k+m} \boldsymbol{R}_{[k, m]}\right) \wedge \Delta=C_{k+m} .
$$

If furthermore $X \in \operatorname{USS}(X)$, this is also true for every $k \in \mathbb{Z}$.

Proof. If $m=1$, we need to show that $\left(C_{k+1} \boldsymbol{R}_{[k, 1]}\right) \wedge \Delta=C_{k+1}$, but we have $\mathbf{c}^{k}(X)=C_{k+1} \Delta^{p} R_{k+1}=C_{k+1} \boldsymbol{R}_{[k, 1]} \Delta^{p}$, where this decomposition is left weighted by definition. So the result is true for $m=1$.

Suppose the result true for $m-1$. This means that

$$
\left(C_{k+m-1} \boldsymbol{R}_{[k, m-1]}\right) \wedge \Delta=C_{k+m-1} .
$$

If we multiply on the left by $R_{k+m-1}$ we get

$$
\left(R_{k+m-1} C_{k+m-1} \boldsymbol{R}_{[k, m-1]}\right) \wedge\left(R_{k+m-1} \Delta\right)=R_{k+m-1} C_{k+m-1} .
$$

Notice that $\Delta \preccurlyeq R_{k+m-1} \Delta$, hence if we consider the maximal simple prefix of each element in the above equality, we obtain

$$
\left(R_{k+m-1} C_{k+m-1} \boldsymbol{R}_{[k, m-1]}\right) \wedge \Delta=\left(R_{k+m-1} C_{k+m-1}\right) \wedge \Delta .
$$

On the other hand, recall that

$$
\left(\Delta^{p} R_{k+m-1}\right) C_{k+m-1}=\mathrm{c}^{k+m-1}(X)=C_{k+m}\left(\Delta^{p} R_{k+m}\right),
$$

whence $R_{k+m-1} C_{k+m-1}=\tau^{p}\left(C_{k+m}\right) R_{k+m}$, where $\tau^{p}\left(C_{k+m}\right)$ is the maximal simple prefix of this element. Therefore, one has

$$
\left(R_{k+m-1} C_{k+m-1} \boldsymbol{R}_{[k, m-1]}\right) \wedge \Delta=\left(R_{k+m-1} C_{k+m-1}\right) \wedge \Delta=\tau^{p}\left(C_{k+m}\right) .
$$

Now notice that

$$
\Delta^{p} R_{k+m-1} C_{k+m-1} \boldsymbol{R}_{[k, m-1]}=C_{k+m} \Delta^{p} R_{k+m} \boldsymbol{R}_{[k, m-1]}=C_{k+m} \boldsymbol{R}_{[k, m]} \Delta^{p} .
$$


This means that

$$
R_{k+m-1} C_{k+m-1} \boldsymbol{R}_{[k, m-1]}=\tau^{p}\left(C_{k+m} \boldsymbol{R}_{[k, m]}\right),
$$

and we just showed that its maximal simple prefix is precisely $\tau^{p}\left(C_{k+m}\right)$. Applying $\tau^{-p}$ to this element, one obtains $\left(C_{k+m} \boldsymbol{R}_{[k, m]}\right) \wedge \Delta=C_{k+m}$, as we wanted to show.

The proof for $X \in \operatorname{USS}(X)$ and $k<0$ is the same.

It will possibly help to understand the situation if we extract a particular case from the above result, assuming that $X \in \operatorname{USS}(X)$ :

Lemma 2.7. Let $X \in \operatorname{USS}(X)$ with $\inf (X)=p$ and $\ell(X) \geq 1$. For every $m \geq 1$, one has

$$
\left(C_{1} \boldsymbol{R}_{[1-m, m]}\right) \wedge \Delta=C_{1}
$$

In other words, the biggest simple prefix of

$$
C_{1} \tau^{-p}\left(R_{1}\right) \tau^{-2 p}\left(R_{0}\right) \tau^{-3 p}\left(R_{-1}\right) \ldots \tau^{-m p}\left(R_{2-m}\right)
$$

is $C_{1}$, no matter how big is $m$.

We can now show that the decomposition $\boldsymbol{C}_{m} \boldsymbol{R}_{m}$ is left weighted. This will actually be a particular case of the following stronger result.

Proposition 2.8. Let $X \in \operatorname{USS}(X)$ with $\ell(X) \geq 1$. With the above notation, for every $k, l, m, n \in \mathbb{Z}$, such that $m, n \geq 1$ and $k+m=l+n$, the decomposition $\boldsymbol{C}_{[k, m]} \boldsymbol{R}_{[l, n]}$ is left weighted. That is, $\varphi\left(\boldsymbol{C}_{[k, m]}\right) \iota\left(\boldsymbol{R}_{[l, n]}\right) \wedge \Delta=\varphi\left(\boldsymbol{C}_{[k, m]}\right)$.

Proof. We proceed by induction on $m$. If $m=1$, one has $\boldsymbol{C}_{[k, 1]} \boldsymbol{R}_{[l, n]}=C_{k+1} \boldsymbol{R}_{[l, n]}$. Since $k+1=l+n$ by hypothesis, it follows from Lemma 2.6 that the biggest simple prefix of this element is precisely $C_{k+1}$. Hence $\boldsymbol{C}_{[k, 1]} \boldsymbol{R}_{[l, n]}$ is left weighted, and the result is true for $m=1$.

Now consider $k, l, m, n$ as above, with $m>1$, and suppose the result true for $m-1$. This implies that $\boldsymbol{C}_{[k, m-1]} \boldsymbol{R}_{[l-1, n]}$ is left weighted, since $k, l-1, m-1, n$ satisfy the required hypothesis. (Notice that if we had required $k, l \geq 0$, we would not have been able to apply the induction hypothesis here, since we could have had $l-1<0$; This is why we require $X \in \operatorname{USS}(X)$ and not only in $\operatorname{SSS}(X)$.)

Let $\boldsymbol{C}_{[k, m-1]}=\Delta^{q} y_{1} \ldots y_{s}$ and $\boldsymbol{R}_{[l-1, n]}=z_{1} \ldots z_{t}$ in left normal form. Then $\boldsymbol{C}_{[k, m-1]} \boldsymbol{R}_{[l-1, n]}=\Delta^{q} y_{1} \ldots y_{s} z_{1} \ldots z_{t}$ is in left normal form as written, by induction hypothesis. Now multiply this element on the right by $\tau^{-p n}\left(C_{l}\right)$. By the normal form algorithm, since $\Delta^{q} y_{1} \ldots y_{s} z_{1} \ldots z_{t}$ is already in left normal form and $\tau^{-p n}\left(C_{l}\right)$ is a simple element, then the left normal form of $\Delta^{q} y_{1} \ldots y_{s} z_{1} \ldots z_{t} \tau^{-p n}\left(C_{l}\right)$ is computed by applying $s+t$ local transformations to pairs of consecutive factors, starting by the rightmost pair. When we apply the first $t$ transformations, we obtain the left 
normal form of $z_{1} \ldots z_{t} \tau^{-p n}\left(C_{l}\right)=\boldsymbol{R}_{[l-1, n]} \tau^{-p n}\left(C_{l}\right)$. By Lemma 2.3, this element is equal to $C_{l+n} \boldsymbol{R}_{[l, n]}=C_{k+m} \boldsymbol{R}_{[l, n]}$. Moreover, by Lemma 2.6, $C_{k+m}$ is the biggest simple prefix of this element. Hence the left normal form of $z_{1} \ldots z_{t} \tau^{-p n}\left(C_{l}\right)$ has the form $C_{k+m} z_{1}^{\prime} \ldots z_{t}^{\prime}$, where $z_{1}^{\prime} \ldots z_{t}^{\prime}=\boldsymbol{R}_{[l, n]}$. We then have

$$
\boldsymbol{C}_{[k, m-1]} \boldsymbol{R}_{[l-1, n]} \tau^{-p n}\left(C_{l}\right)=\boldsymbol{C}_{[k, m-1]} C_{k+m} \boldsymbol{R}_{[l, n]}=\Delta^{q} y_{1} \ldots y_{s} C_{k+m} z_{1}^{\prime} \ldots z_{t}^{\prime},
$$

where the last $t+1$ factors in the latter decomposition are in left normal form.

If we continue applying the normal form algorithm, we perform $s$ local transformations to the element $y_{1} \ldots y_{s} C_{k+m}$, which is equal to $\boldsymbol{C}_{[k, m-1]} C_{k+m}=\boldsymbol{C}_{[k, m]}$. Since the resulting factorization of $\boldsymbol{C}_{[k, m]} \boldsymbol{R}_{[l, n]}$ is in left normal form by construction, it follows that $\varphi\left(\boldsymbol{C}_{[k, m]}\right) \iota\left(\boldsymbol{R}_{[l, n]}\right)$ is left weighted, as we wanted to show.

This result implies one of the strongest relations between cyclings and powers of an element $X$ in its ultra summit set:

Theorem 2.9. Let $X \in \operatorname{USS}(X)$ with $\inf (X)=p$ and $\ell(X)>1$. For every $m \geq 1$, the product of the first $m$ factors (including $\Delta$ 's) in the left normal form of $X^{m} \Delta^{-m p}$ is equal to $\boldsymbol{C}_{m}$. That is,

$$
\left(X^{m} \Delta^{-m p}\right) \wedge \Delta^{m}=C_{m} .
$$

In particular, $\iota\left(X^{m}\right)=\iota\left(\boldsymbol{C}_{m}\right)$.

Proof. The first claim is a straightforward consequence of the previous result and Lemma 2.4, since $X^{m} \Delta^{-m p}=\boldsymbol{C}_{m} \boldsymbol{R}_{m}$, where the latter decomposition is left weighted and $\sup \left(\boldsymbol{C}_{m}\right)=m$.

The second claim follows from the fact that $\iota(Y)=\iota\left(Y \Delta^{t}\right)$ for every $Y \in G$ and every $t \in \mathbb{Z}$. Hence $\iota\left(X^{m}\right)=\iota\left(X^{m} \Delta^{-m p}\right)=\iota\left(\boldsymbol{C}_{m} \boldsymbol{R}_{m}\right)$. Since the latter decomposition is left weighted, and $\ell\left(\boldsymbol{C}_{m}\right)>0$, it follows that $\iota\left(X^{m}\right)=\iota\left(\boldsymbol{C}_{m}\right)$, as we wanted to show.

Corollary 2.10. For $X \in \operatorname{USS}(X)$ and $m \geq 1$, one has

$$
\inf \left(X^{m}\right)=m \inf (X)+\inf \left(\boldsymbol{C}_{m}\right) .
$$

In particular, the unexpected $\Delta^{\prime} s$ in $X^{m}$ are determined entirely by the $\boldsymbol{C}_{m}$ part of the normal form of $X^{m}$.

Proof. Since we know by Lemma 2.4 that $\ell\left(\boldsymbol{C}_{m}\right)>0$, it follows from Theorem 2.9 that all $\Delta$ 's in the left normal form of $X^{m}$ can be seen in $\Delta^{p m} \tau^{p m}\left(\boldsymbol{C}_{m}\right)$, where $p=\inf (X)$. Hence $\inf \left(X^{m}\right)=p m+\inf \left(\boldsymbol{C}_{m}\right)$, and the result follows.

We end this section with an immediate corollary concerning how the infimum and supremum of an element, in its ultra summit set, behave when one raises the element to some power. This is related to the translation number of the element (see [32]). Notice that the following result is closely related to Proposition 3.6 in [30]. 
Corollary 2.11. Let $X \in \operatorname{USS}(X)$. For every $m \geq 1$, one has

$$
\inf \left(X^{m}\right)+\inf (X) \leq \inf \left(X^{m+1}\right) \leq \inf \left(X^{m}\right)+\inf (X)+1 .
$$

If $X^{-1} \in \operatorname{USS}\left(X^{-1}\right)$, then

$$
\sup \left(X^{m}\right)+\sup (X)-1 \leq \sup \left(X^{m+1}\right) \leq \sup \left(X^{m}\right)+\sup (X) .
$$

Proof. Let $p=\inf (X)$. By the previous corollary, $\inf \left(X^{m}\right)=p m+\inf \left(\boldsymbol{C}_{m}\right)$ and $\inf \left(X^{m+1}\right)=p(m+1)+\inf \left(\boldsymbol{C}_{m+1}\right)$. Hence, the first inequality will be true if and only if

$$
\inf \left(\boldsymbol{C}_{m}\right) \leq \inf \left(\boldsymbol{C}_{m+1}\right) \leq \inf \left(\boldsymbol{C}_{m}\right)+1 .
$$

But $C_{m+1}=C_{m} C_{m+1}$, where $C_{m+1}$ is a simple element. The result then follows from the following well know fact, which is a direct consequence of the normal form algorithm: If an element in a Garside group is multiplied by a simple element, then its infimum either is preserved or is increased by one.

The second inequality is equivalent to the first one, since $\sup (X)=-\inf \left(X^{-1}\right)$ by Corollary 1.6.

2.3. The absolute initial and final factors. In this section we will define some simple factors related to an element $X \in \operatorname{USS}(X)$. They are defined in terms of the cycling elements $C_{i}$, but they are closely related to powers of $X$, as we will see. We called them the absolute initial and final factors of $X$.

In general, the absolute initial and final factors are related to, but do not coincide with, the initial and final factors of $X$. Nevertheless we will see that, if $X$ has a rigid power $X^{m}$, the absolute initial and final factors of $X$ coincide with the initial and final factors of $X^{m}$.

Suppose that $X \in \operatorname{USS}(X)$. We saw in Lemma 2.5 that

$$
\varphi\left(\boldsymbol{C}_{[k, m]}\right)=\varphi\left(C_{k+1} \ldots C_{k+m}\right) \succcurlyeq \varphi\left(\mathbf{c}^{k+m}(X)\right),
$$

where this is true for every $m \geq 1$ and every $k \in \mathbb{Z}$. This implies a very interesting fact: if we fix the number $k+m$ and take different values of $k$, that is, if we start with $C_{k+m}$ and multiply it on the left by $C_{k+m-1}$, then by $C_{k+m-2}$, etc., then the final factor of each of the resulting elements is a left multiple of $\varphi\left(\mathbf{c}^{k+m}(X)\right)$. For instance, if we take $k+m=0$, we have

$$
\varphi\left(C_{-m+1} C_{-m+2} \ldots C_{-1} C_{0}\right) \geqslant \varphi(X),
$$

for every $m \geq 1$.

In the same way, by Lemma 2.5 we know that

$$
\iota\left(\boldsymbol{R}_{[k, m]}\right)=\iota\left(\tau^{-p}\left(R_{k+m}\right) \tau^{-2 p}\left(R_{k+m-1}\right) \ldots \tau^{-m p}\left(R_{k+1}\right)\right) \preccurlyeq C_{k+m+1}
$$


for every $m \geq 1$ and every $k \in \mathbb{Z}$, where $p=\inf (X)$. Hence, if we fix $k+m$, say $k+m=0$, we have (recall that $\left.C_{1}=\iota(X)\right)$

$$
\iota\left(\tau^{-p}\left(R_{0}\right) \tau^{-2 p}\left(R_{-1}\right) \ldots \tau^{-m p}\left(R_{-m+1}\right)\right) \preccurlyeq \iota(X),
$$

for every $m \geq 1$. In the particular case in which $\inf (X)=p=0$, this formula is even more similar to the above one, since one has

$$
\iota\left(R_{0} R_{-1} \ldots R_{-m+1}\right) \preccurlyeq \iota(X),
$$

for every $m \geq 1$.

But it is even more interesting to relate the values of $\varphi\left(C_{-m+1} C_{-m+2} \ldots C_{-1} C_{0}\right) \quad$ and of $\quad \iota\left(\tau^{-p}\left(R_{0}\right) \tau^{-2 p}\left(R_{-1}\right) \ldots \tau^{-m p}\left(R_{-m+1}\right)\right)$, respectively, for different values of $m$. It turns out that they form ordered chains with respect to $\succcurlyeq$ and $\preccurlyeq$, respectively, as shown in the following result.

Proposition 2.12. Let $X \in \operatorname{USS}(X)$ with $\ell(X)>1$. For every $k, m \in \mathbb{Z}$ with $m>0$, one has

$$
\varphi\left(\boldsymbol{C}_{[k, m]}\right) \succcurlyeq \varphi\left(\boldsymbol{C}_{[k-1, m+1]}\right) \text { and } \iota\left(\boldsymbol{R}_{[k, m]}\right) \preccurlyeq \iota\left(\boldsymbol{R}_{[k-1, m+1]}\right) .
$$

In other words, for every $k \in \mathbb{Z}$ there are chains

$$
\varphi\left(\boldsymbol{C}_{[k, 1]}\right) \succeq \varphi\left(\boldsymbol{C}_{[k-1,2]}\right) \succeq \varphi\left(\boldsymbol{C}_{[k-2,3]}\right) \succeq \cdots
$$

and

$$
\iota\left(\boldsymbol{R}_{[k, 1]}\right) \preccurlyeq \iota\left(\boldsymbol{R}_{[k-1,2]}\right) \preccurlyeq \iota\left(\boldsymbol{R}_{[k-2,3]}\right) \preccurlyeq \cdots .
$$

Proof. We know that $\sup \left(\boldsymbol{C}_{[k, m]}\right)=m$ and $\sup \left(\boldsymbol{C}_{[k-1, m+1]}\right)=m+1$. Moreover, $\boldsymbol{C}_{[k-1, m+1]}=C_{k} C_{k+1} \ldots C_{k+m}=C_{k} \boldsymbol{C}_{[k, m]}$. Hence, if we write $\boldsymbol{C}_{[k, m]}=$ $\Delta^{q} c_{1} \ldots c_{s}$ in left normal form (where $q+s=m$ and $s>0$ ), then $\varphi\left(\boldsymbol{C}_{[k, m]}\right)=c_{s} \succeq$ $\varphi\left(C_{k} \Delta^{q} c_{1} \ldots c_{s}\right)=\varphi\left(\boldsymbol{C}_{[k-1, m+1]}\right)$.

On the other hand, let $p=\inf (X)$. One has

$$
\begin{aligned}
\boldsymbol{R}_{[k-1, m+1]} & =\tau^{-p}\left(R_{k+m}\right) \tau^{-2 p}\left(R_{k+m-1}\right) \ldots \tau^{-m p}\left(R_{k+1}\right) \tau^{-(m+1) p}\left(R_{k}\right) \\
& =\boldsymbol{R}_{[k, m]} \cdot \tau^{-(m+1) p}\left(R_{k}\right),
\end{aligned}
$$

that is, $\boldsymbol{R}_{[k, m]} \preccurlyeq \boldsymbol{R}_{[k-1, m+1]}$. As we know that $\inf \left(\boldsymbol{R}_{[k, m]}\right)=\inf \left(\boldsymbol{R}_{[k-1, m+1]}\right)=0$, it follows that $\iota\left(\boldsymbol{R}_{[k, m]}\right) \preccurlyeq \iota\left(\boldsymbol{R}_{[k-1, m+1]}\right)$.

Since the chains given by the above proposition consist of proper simple elements, we know that the chains must stabilize. But we will furthermore show that they stabilize fast. More precisely, they stabilize exactly at the first repetition. Moreover, the corresponding chains for all elements in $\mathcal{O}(X)$ stabilize at the same time. This is proved by the next four lemmas and the proposition that follows them. 
Lemma 2.13. Let $X \in \operatorname{USS}(X)$ with $\ell(X)>1$ and $m>0$. If $\varphi\left(\boldsymbol{C}_{[k, m]}\right)=$ $\varphi\left(\boldsymbol{C}_{[k-1, m+1]}\right)$ for some $k \in \mathbb{Z}$, then $\varphi\left(\boldsymbol{C}_{[i, m]}\right)=\varphi\left(\boldsymbol{C}_{[i-1, m+1]}\right)$ for every $i \in \mathbb{Z}$.

Proof. Since $X$ belongs to a closed orbit under cycling, the sequences $\left\{\boldsymbol{C}_{[i, m]}\right\}_{i \in \mathbb{Z}}$ and $\left\{\boldsymbol{C}_{[i, m+1]}\right\}_{i \in \mathbb{Z}}$ are periodic, hence it suffices to show the case $i=k+1$.

Note that we can reinterpret the property $\varphi\left(\boldsymbol{C}_{[k, m]}\right)=\varphi\left(\boldsymbol{C}_{[k-1, m+1]}\right)$, that is, $\varphi\left(C_{k+1} \ldots C_{k+m}\right)=\varphi\left(C_{k} \ldots C_{k+m}\right)$, as follows. Since $\sup \left(C_{k+1} \ldots C_{k+m}\right)=m$ and $\sup \left(C_{k} \ldots C_{k+m}\right)=m+1$, their final factors coincide if and only if the first $m-1$ factors of the first element, multiplied on the left by $C_{k}$, coincide with the first $m$ factors of the second element. In other words,

$$
\left(C_{k} \ldots C_{k+m}\right) \wedge\left(C_{k} \Delta^{m-1}\right)=\left(C_{k} \ldots C_{k+m}\right) \wedge \Delta^{m} .
$$

We can now apply Gebhardt's transport [24] to the whole equality. We know that the transport of $\Delta^{m}$ is $\Delta^{m}$. Notice that the transport of $C_{k}$ (based at $\left.\mathbf{c}^{k-1}(X)\right)$ is $C_{k+1}$. This implies, by recurrence, that the transport of $C_{k} \ldots C_{k+m}$ is $C_{k+1} \ldots C_{k+m+1}$, and also that the transport of $C_{k} \Delta^{m-1}$ is $C_{k+1} \Delta^{m-1}$. Since the transport preserves greatest common divisors, the transport of the above equality yields

$$
\left(C_{k+1} \ldots C_{k+m+1}\right) \wedge\left(C_{k+1} \Delta^{m-1}\right)=\left(C_{k+1} \ldots C_{k+m+1}\right) \wedge \Delta^{m},
$$

that is, $\varphi\left(\boldsymbol{C}_{[k+1, m]}\right)=\varphi\left(\boldsymbol{C}_{[k, m+1]}\right)$, and the result is shown.

Lemma 2.14. Let $X \in \operatorname{USS}(X)$ with $\ell(X)>1$. If $\varphi\left(\boldsymbol{C}_{[k, m]}\right)=\varphi\left(\boldsymbol{C}_{[k-1, m+1]}\right)$ for some $k, m \in \mathbb{Z}$ with $m>0$, then $\varphi\left(\boldsymbol{C}_{[i, j]}\right)=\varphi\left(\boldsymbol{C}_{[i-1, j+1]}\right)$ for every $i \in \mathbb{Z}$ and every $j \geq m$.

Proof. We know by Lemma 2.13 that $\varphi\left(\boldsymbol{C}_{[i, m]}\right)=\varphi\left(\boldsymbol{C}_{[i-1, m+1]}\right)$ for every $i \in \mathbb{Z}$. We just need to be able to increase the second subindex. But if $\varphi\left(C_{i+1} \ldots C_{i+m}\right)=$ $\varphi\left(C_{i} \ldots C_{i+m}\right) \neq \Delta$, and we multiply both elements on the right by $C_{i+m+1}$, since we know that no unexpected decrease of supremum will happen $\left(\sup \left(\boldsymbol{C}_{[i, m+1]}\right)=m+1\right)$, it follows that

$$
\begin{aligned}
\varphi\left(C_{i+1} \ldots C_{i+m} C_{i+m+1}\right) & =\varphi\left(\varphi\left(C_{i+1} \ldots C_{i+m}\right) C_{i+m+1}\right) \\
& =\varphi\left(\varphi\left(C_{i} \ldots C_{i+m}\right) C_{i+m+1}\right) \\
& =\varphi\left(C_{i} \ldots C_{i+m} C_{i+m+1}\right) .
\end{aligned}
$$

Hence $\varphi\left(\boldsymbol{C}_{[i, m+1]}\right)=\varphi\left(\boldsymbol{C}_{[i-1, m+2]}\right)$ for every $i \in \mathbb{Z}$. By induction on $m$, it follows that $\varphi\left(\boldsymbol{C}_{[i, j]}\right)=\varphi\left(\boldsymbol{C}_{[i-1, j+1]}\right)$ for every $j \geq m$, as we wanted to show.

The analogous results can now be shown for the chain involving prefixes of $\boldsymbol{R}_{[k, m]}$.

Lemma 2.15. Let $X \in \operatorname{USS}(X)$ with $\ell(X)>1$ and $m \geq 1$. If $\iota\left(\boldsymbol{R}_{[k, m]}\right)=$ $\iota\left(\boldsymbol{R}_{[k-1, m+1]}\right)$ for some $k \in \mathbb{Z}$, then $\iota\left(\boldsymbol{R}_{[i, m]}\right)=\iota\left(\boldsymbol{R}_{[i-1, m+1]}\right)$ for every $i \in \mathbb{Z}$. 
Proof. As above, since $X$ belongs to a closed orbit under cycling, it suffices to show the case $i=k+1$. We want to reinterpret the equality $\iota\left(\boldsymbol{R}_{[k, m]}\right)=\iota\left(\boldsymbol{R}_{[k-1, m+1]}\right)$. If we recall that $\left(\mathbf{c}^{k}(X)\right)^{m}=\boldsymbol{C}_{[k, m]} \boldsymbol{R}_{[k, m]} \Delta^{m p}$ where $p=\inf (X)$, and that $\inf \left(\boldsymbol{R}_{[k, m]}\right)=0$, then we see that

$$
\begin{aligned}
\left(\mathbf{c}^{k}(X)\right)^{m} \wedge\left(\boldsymbol{C}_{[k, m]} \Delta^{m p+1}\right) & =\boldsymbol{C}_{[k, m]} \iota\left(\boldsymbol{R}_{[k, m]}\right) \Delta^{m p} \\
& =C_{k+1} \ldots C_{k+m} \iota\left(\boldsymbol{R}_{[k, m]}\right) \Delta^{m p}
\end{aligned}
$$

In the same way, we obtain

$$
\begin{aligned}
\left(\mathbf{c}^{k-1}(X)\right)^{m+1} \wedge\left(\boldsymbol{C}_{[k-1, m+1]} \Delta^{(m+1) p+1}\right) & =\boldsymbol{C}_{[k-1, m+1]} \iota\left(\boldsymbol{R}_{[k-1, m+1]}\right) \Delta^{(m+1) p} \\
& =C_{k} \ldots C_{k+m} \iota\left(\boldsymbol{R}_{[k-1, m+1]}\right) \Delta^{m p+p}
\end{aligned}
$$

Therefore the equality $\iota\left(\boldsymbol{R}_{[k, m]}\right)=\iota\left(\boldsymbol{R}_{[k-1, m+1]}\right)$ can be rewritten as follows:

$$
C_{k}\left[\left(\mathbf{c}^{k}(X)\right)^{m} \wedge\left(C_{[k, m]} \Delta^{m p+1}\right)\right] \Delta^{p}=\left(\mathbf{c}^{k-1}(X)\right)^{m+1} \wedge\left(C_{[k-1, m+1]} \Delta^{(m+1) p+1}\right) .
$$

If we apply Gebhardt's transport to the whole equality, it follows that

$$
\left.C_{k+1}\left(\mathbf{c}^{k+1}(X)\right)^{m} \wedge\left(C_{[k+1, m]} \Delta^{m p+1}\right)\right] \Delta^{p}=\left(\mathbf{c}^{k}(X)\right)^{m+1} \wedge\left(C_{[k, m+1]} \Delta^{(m+1) p+1}\right),
$$

hence $\iota\left(\boldsymbol{R}_{[k+1, m]}\right)=\iota\left(\boldsymbol{R}_{[k, m+1]}\right)$, and the result is shown.

Lemma 2.16. Let $X \in \mathrm{USS}(X)$ with $\ell(X)>1$. If $\iota\left(\boldsymbol{R}_{[k, m]}\right)=\iota\left(\boldsymbol{R}_{[k-1, m+1]}\right)$ for some $k, m \in \mathbb{Z}$ with $m>0$, then $\iota\left(\boldsymbol{R}_{[i, j]}\right)=\iota\left(\boldsymbol{R}_{[i-1, j+1]}\right)$ for every $i \in \mathbb{Z}$ and every $j \geq m$.

Proof. By Lemma $2.15 \iota\left(\boldsymbol{R}_{[i, m]}\right)=\iota\left(\boldsymbol{R}_{[i-1, m+1]}\right)$ for every $i \in \mathbb{Z}$. We just need to be able to increase the second subindex. But $\iota\left(\boldsymbol{R}_{[i, m]}\right)=\iota\left(\boldsymbol{R}_{[i-1, m+1]}\right)$ is equivalent to $\iota\left(\left(\Delta^{p} R_{i+m}\right)\left(\Delta^{p} R_{i+m-1}\right) \ldots\left(\Delta^{p} R_{i+1}\right)\right)=\iota\left(\left(\Delta^{p} R_{i+m}\right)\left(\Delta^{p} R_{i+m-1}\right) \ldots\left(\Delta^{p} R_{i}\right)\right)$, where $p=\inf (X)$. If we multiply both elements on the left by $\Delta^{p} R_{i+m+1}$, since we know that there is no unexpected increase of infimum $\left(\inf \left(\boldsymbol{R}_{[i, m+1]}\right)=0\right)$, it follows that

$$
\begin{aligned}
\iota\left(\left(\Delta^{p} R_{i+m+1}\right)\left(\Delta^{p} R_{i+m}\right) \ldots\left(\Delta^{p} R_{i+1}\right)\right) \\
\quad=\iota\left(\left(\Delta^{p} R_{i+m+1}\right) \iota\left(\left(\Delta^{p} R_{i+m}\right) \ldots\left(\Delta^{p} R_{i+1}\right)\right)\right) \\
\quad=\iota\left(\left(\Delta^{p} R_{i+m+1}\right) \iota\left(\left(\Delta^{p} R_{i+m}\right) \ldots\left(\Delta^{p} R_{i}\right)\right)\right) \\
\quad=\iota\left(\left(\Delta^{p} R_{i+m}\right)\left(\Delta^{p} R_{i+m-1}\right) \ldots\left(\Delta^{p} R_{i}\right)\right) .
\end{aligned}
$$

Hence $\iota\left(\boldsymbol{R}_{[i, m+1]}\right)=\iota\left(\boldsymbol{R}_{[i-1, m+2]}\right)$ for every $i \in \mathbb{Z}$. By induction on $m$, it follows that $\iota\left(\boldsymbol{R}_{[i, j]}\right)=\iota\left(\boldsymbol{R}_{[i-1, j+1]}\right)$ for every $j \geq m$, as we wanted to show. 
Proposition 2.17. Let $X \in \operatorname{USS}(X)$ with $\ell(X)>1$. Given $k \in \mathbb{Z}$, the chain

$$
\varphi\left(\boldsymbol{C}_{[k, 1]}\right) \succcurlyeq \varphi\left(\boldsymbol{C}_{[k-1,2]}\right) \succcurlyeq \varphi\left(\boldsymbol{C}_{[k-2,3]}\right) \succcurlyeq \cdots
$$

stabilizes whenever $\varphi\left(\boldsymbol{C}_{[k-j+1, j]}\right)=\varphi\left(\boldsymbol{C}_{[k-j, j+1]}\right)$, and this happens for some $j<\|\Delta\|$. Moreover, for all $i \in \mathbb{Z}$, the analogous chains starting at $\varphi\left(\boldsymbol{C}_{[i, 1]}\right)$ stabilize at the same value of $j$. Also, the chain

$$
\iota\left(\boldsymbol{R}_{[k, 1]}\right) \preccurlyeq \iota\left(\boldsymbol{R}_{[k-1,2]}\right) \preccurlyeq \iota\left(\boldsymbol{R}_{[k-2,3]}\right) \preccurlyeq \cdots
$$

stabilizes whenever $\iota\left(\boldsymbol{R}_{[k-j+1, j]}\right)=\iota\left(\boldsymbol{R}_{[k-j, j+1]}\right)$, and this happens for some $j<\|\Delta\|$. Moreover, for all $i \in \mathbb{Z}$, the analogous chains starting at $\iota\left(\boldsymbol{R}_{[i, 1]}\right)$ stabilize at the same value of $j$.

Proof. By Lemma 2.14, all chains of final factors stabilize whenever $\varphi\left(\boldsymbol{C}_{[k-j+1, j]}\right)=$ $\varphi\left(\boldsymbol{C}_{[k-j, j+1]}\right)$ for some $j$. Up to that point, the chains must be made of strict inequalities. But the maximal length of such a chain (formed by nontrivial simple elements) is bounded by the length of $\Delta$. The proof for the sequences of initial factors is identical.

Definition 2.18. Given $X \in \operatorname{USS}(X)$ with $\ell(X)>1$, we define the absolute final factor $F(X)$ of $X$ as the factor in which the above descending chain stabilizes, for $k=-1$, that is,

$$
F(X)=\varphi\left(\boldsymbol{C}_{[-m, m]}\right)
$$

for $m \geq\|\Delta\|-1$. In other words, $F(X)=\varphi\left(C_{-m+1} C_{-m+2} \ldots C_{-1} C_{0}\right)$ for $m$ big enough.

Definition 2.19. Given $X \in \operatorname{USS}(X)$ with $\ell(X)>1$, we define the absolute initial factor $I(X)$ of $X$ as the factor in which the above ascending chain stabilizes, for $k=-1$, that is,

$$
I(X)=\iota\left(\boldsymbol{R}_{[-m, m]}\right)
$$

for $m \geq\|\Delta\|-1$. In other words, $I(X)=\iota\left(\tau^{-p}\left(R_{0}\right) \tau^{-2 p}\left(R_{-1}\right) \ldots \tau^{-m p}\left(R_{-m+1}\right)\right)$, or alternatively $I(X)=\iota\left(\left(\Delta^{p} R_{0}\right)\left(\Delta^{p} R_{-1}\right) \ldots\left(\Delta^{p} R_{-m+1}\right)\right)$, for $m$ big enough, where $p=\inf (X)$.

Proposition 2.20. Given $X \in \operatorname{USS}(X)$ with $\ell(X)>1$, the decomposition $F(X) I(X)$ is left weighted as written.

Proof. This is an immediate consequence of Proposition 2.8, since

$$
F(X) I(X)=\varphi\left(\boldsymbol{C}_{[-m, m]}\right) \iota\left(\boldsymbol{R}_{[-m, m]}\right)
$$

for $m$ big enough. 
We have seen at the beginning of this section that $F(X) \succcurlyeq \varphi(X)$ and $I(X) \preccurlyeq \iota(X)$. But we will see now that the absolute factors are also related to the initial and final factors of powers of $X$.

Proposition 2.21. Let $X \in \operatorname{USS}(X)$ with $\inf (X)=p$ and $\ell(X)>1$. For every $m \geq 1$ such that $X^{m} \in \operatorname{SSS}\left(X^{m}\right)$, one has:

- $F(X) \succcurlyeq \varphi\left(X^{m}\right)$.

- $I(X) \preccurlyeq \iota\left(X^{m}\right)$.

Proof. The case $m=1$ is a straightforward consequence of Lemma 2.5, for $k=-m$.

Suppose that $m>1$. We know from Theorem 2.9 that the left normal form of

$$
\left(\mathbf{c}^{-m}(X)\right)^{m}=\left(C_{-m+1} \ldots C_{0}\right) X^{m}\left(C_{0}^{-1} \ldots C_{-m+1}^{-1}\right)
$$

is equal to

$$
\Delta^{p m+q} y_{1} \ldots y_{s} z_{1} \ldots z_{t},
$$

where $\Delta^{q} y_{1} \ldots y_{s}=\tau^{p m}\left(C_{-m+1} \ldots C_{0}\right)$ and $q+s=m$. If we conjugate this element by $C_{-m+1} \ldots C_{0}$ we obtain

$$
X^{m}=\Delta^{p m} z_{1} \ldots z_{t} \Delta^{q} \tau^{-p m}\left(y_{1} \ldots y_{s}\right) .
$$

But if $X^{m} \in \operatorname{SSS}\left(X^{m}\right)$, we also have $Y^{m} \in \operatorname{SSS}\left(X^{m}\right)$ for every $Y$ in the cycling orbit of $X$ : Indeed, $Y^{m}=\left(X^{\boldsymbol{C}_{t}}\right)^{m}=\left(X^{m}\right)^{\boldsymbol{C}_{t}}$ for some $t$, where $\boldsymbol{C}_{t}=\left(X^{m} \Delta^{-m p}\right) \wedge \Delta^{t}$ by Theorem 2.9. Since $X^{m} \Delta^{-m p}$ and $\Delta^{t}$ conjugate $X^{m}$ to elements in their super summit sets (namely $\tau^{-m p}\left(X^{m}\right)$ and $\tau^{t}\left(X^{m}\right)$, respectively), it follows by Theorem 1.12 that $Y^{m}=\left(X^{m}\right) \boldsymbol{C}_{t} \in \operatorname{SSS}\left(X^{m}\right)$. In particular $\left(\mathbf{c}^{-m}(X)\right)^{m} \in \operatorname{SSS}\left(X^{m}\right)$, hence $\ell\left(X^{m}\right)=\ell\left(\left(\mathbf{c}^{-m}(X)\right)^{m}\right)=s+t$. Since the above decomposition of $X^{m}$ has precisely $s+t$ non- $\Delta$ factors, and the final one is $\tau^{-p m}\left(y_{s}\right)$, it follows that $\tau^{-p m}\left(y_{s}\right) \geqslant \varphi\left(X^{m}\right)$. That is, $\varphi\left(C_{-m+1} \ldots C_{0}\right) \geqslant \varphi\left(X^{m}\right)$.

Notice that we can apply the same reasoning to every element in the cycling orbit of $X$, in particular to $\mathbf{c}^{-m}(X)$. It follows that $\varphi\left(C_{-2 m+1} \ldots C_{-m}\right) \geqslant \varphi\left(\left(\mathbf{c}^{-m}(X)\right)^{m}\right)=$ $z_{t}$. Hence, since multiplying $C_{i}$ 's never decreases the supremum, one has

$$
\begin{aligned}
\varphi\left(C_{-2 m+1} \ldots C_{-m} C_{-m+1} \ldots C_{0}\right) & =\varphi\left(\varphi\left(C_{-2 m+1} \ldots C_{-m}\right) C_{-m+1} \ldots C_{0}\right) \\
& \succcurlyeq \varphi\left(z_{t} \Delta^{q} \tau^{-p m}\left(y_{1} \ldots y_{s}\right)\right)=\varphi\left(X^{m}\right) .
\end{aligned}
$$

Applying the same reasoning once more, we can obtain by induction on $k$ that $\varphi\left(C_{-k m+1} \ldots C_{0}\right) \geqslant \varphi\left(X^{m}\right)$ for every $k \geq 1$. When $k$ is big enough so that $k m \geq\|\Delta\|-1$, this implies $F(X) \geqslant \varphi\left(X^{m}\right)$, as we wanted to show.

The relation $I(X) \preccurlyeq \iota\left(X^{m}\right)$ is shown in a similar way. Since $\tau^{-p m}\left(z_{1} \ldots z_{t}\right)=$ $\boldsymbol{R}_{[-m, m]}$, from the above decomposition of $X^{m}$ is follows that $\iota\left(\boldsymbol{R}_{[-m, m]}\right)=$ 
$\tau^{-p m}\left(z_{1}\right) \preccurlyeq \iota\left(X^{m}\right)$. Applying the same reasoning to $\mathbf{c}^{-m}(X)$, is follows that $\iota\left(\boldsymbol{R}_{[-2 m, m]}\right) \preccurlyeq \iota\left(\left(\mathbf{c}^{-m}(X)\right)^{m}\right)=\tau^{-p m-q}\left(y_{1}\right)$. Hence

$$
\begin{aligned}
\iota\left(\boldsymbol{R}_{[-2 m, 2 m]}\right) & =\iota\left(\boldsymbol{R}_{[-m, m]} \tau^{p m}\left(\boldsymbol{R}_{[-2 m, m]}\right)\right) \\
& =\iota\left(\boldsymbol{R}_{[-m, m]} \iota\left(\tau^{p m}\left(\boldsymbol{R}_{[-2 m, m]}\right)\right)\right) \\
& \preccurlyeq \iota\left(\boldsymbol{R}_{[-m, m]} \tau^{-q}\left(y_{1}\right)\right)=\iota\left(\Delta^{p m} z_{1} \ldots z_{t} \Delta^{q} \tau^{-m p}\left(y_{1}\right)\right)=\iota\left(X^{m}\right) .
\end{aligned}
$$

Iterating the same reasoning one shows that $\iota\left(\boldsymbol{R}_{[-k m, k m]}\right) \preccurlyeq \iota\left(X^{m}\right)$ for every $k \geq 1$, and when $k$ is big enough this yields $I(X) \preccurlyeq \iota\left(X^{m}\right)$.

2.4. The stable ultra summit set. We have studied, up to now, how powers and cyclings of $X$ are related under the hypothesis, in most cases, that $X \in \operatorname{USS}(X)$. But this fact does not imply that $X^{m} \in \operatorname{USS}\left(X^{m}\right)$ for every $m \in \mathbb{Z}$, not even for every $m \in \mathbb{N}$. If we want to extract more information from the powers of $X$, it would be desirable that all these powers belonged to their ultra summit sets.

Definition 2.22. Given $X$ in a Garside group $G$, the stable ultra summit set of $X$ is defined as

$$
\operatorname{SU}(X)=\left\{Y \in \operatorname{USS}(X) ; Y^{m} \in \operatorname{USS}\left(X^{m}\right), m \in \mathbb{Z}\right\} .
$$

The first obvious question is whether $\mathrm{SU}(X) \neq \emptyset$.

Proposition 2.23. For every $X \in G$, the set $\mathrm{SU}(X)$ is non-empty.

Proof. We can clearly assume that $\ell(Y)>0$ for every $Y \in \operatorname{USS}(X)$. Given an element $Z=\Delta^{p} z_{1} \ldots z_{r}$, its initial factor $\iota(Z)$ can be described as $Z \Delta^{-p} \wedge \Delta$, because $Z \Delta^{-p}$ is equal to $\tau^{-p}\left(z_{1} \ldots z_{r}\right)$, hence the initial factor of $Z$ is equal to the first factor of $Z \Delta^{-p}$, which is computed by considering its gcd with $\Delta$.

Recall that $\iota(Z)$ is also the conjugating element for cycling. On the other hand, the conjugating element for decycling is $\Delta^{p} z_{1} \ldots z_{r-1}$, which can be described as $Z \wedge \Delta^{p+r-1}$ (even if $p$ is negative).

Therefore, if we want to apply a cycling or a decycling to $Z$, we must conjugate it by $Z \Delta^{-p} \wedge \Delta$ or by $Z \wedge \Delta^{p+r-1}$, respectively.

Now consider an element $X$. For every $Y \in \operatorname{USS}(X)$, define $S(Y)$ to be the set of integers $k$ such that $Y^{k}$ belongs to its USS. Let $V \in \operatorname{USS}(X)$ be such that $S(V)$ is maximal. Such a $V$ exists because $\operatorname{USS}(X)$ is finite. We will see that $S(V)=\mathbb{Z}$, hence $V \in \mathrm{SU}(X)$.

Suppose that $S(V) \neq \mathbb{Z}$. This means that some power of $V$, say $V^{k}$, does not belong to its USS. We would then like to apply cyclings and decyclings to $V^{k}$ to bring it into $\operatorname{USS}\left(X^{k}\right)$. Let us conjugate $V$ by $\iota\left(V^{k}\right)$, to obtain some $W$. In this way, all powers of $V$ will be conjugate by $\iota\left(V^{k}\right)$. In particular, $W^{k}$ will be the cycling of $V^{k}$. Moreover, if some other power of $V$, say $V^{s}$, belongs to its USS, then $W^{s}$ 
also belongs to its USS. Indeed, let $m=\inf \left(V^{k}\right)$. It is clear that $V^{k} \Delta^{-m}$ conjugates $V^{s}$ to an element in its USS, namely $\tau^{-m}\left(V^{s}\right)$. In the same way, $\Delta$ conjugates $V^{s}$ to an element in its USS, $\tau\left(V^{s}\right)$. Therefore, by Theorem 1.16, $\iota\left(V^{k}\right)=\left(V^{k} \Delta^{-m}\right) \wedge \Delta$ conjugates $V^{s}$ to an element $\left(W^{s}\right)$ in its USS.

Therefore, the set $S(W)$ contains $S(V)$, and the power $W^{k}$ is the cycling of $V^{k}$. The same can be done for decycling, since the conjugating element for decycling $V^{k}$ is $V^{k} \wedge \Delta^{t}$ (for some $t$ ). Hence, by suitable conjugations of $V$, we can apply iterated cyclings and decyclings to $V^{k}$, until we obtain a conjugate $Z$ of $V$ such that $S(Z)$ contains $S(V)$, and $Z^{k}$ belongs to its USS. But then $S(Z)$ strictly contains $S(V)$, which contradicts the maximality of $S(V)$. This shows that $\mathrm{SU}(X)$ is non-empty.

Remark. Although we had a different name for the stable ultra summit set, we chose the latter when we learnt about the paper [30], in which the stable super summit set was defined in a similar way as above, but considering $Y^{m} \in \operatorname{SSS}\left(Y^{m}\right)$ for every $m \in \mathbb{N}$. We remark that Proposition 2.23 was made public by the authors at a meeting on braid groups held in Luminy, in June 2005, some months before the appearance of [30]. Notice also that the proof of Proposition 2.23 can be applied to show the non-emptiness of the stable super summit set, using Theorem 1.12 instead of Theorem 1.16. Moreover, one can extend the set of exponents to the whole $\mathbb{Z}$, in both cases. The proof of the non-emptiness of the stable super summit set in [30] is much more involved, and was found independently from ours.

Now notice that the proof of Proposition 2.23 yields an algorithm to compute the set

$$
\left\{Y \in \operatorname{USS}(X) ; Y^{m} \in \operatorname{USS}\left(X^{m}\right), m \in[A, B]\right\}
$$

for every pair of integers $A<B$, that is, we can assume that $X^{m}$ belongs to its ultra summit set for all integers between $A$ and $B$. A priori, no matter how big is the interval $[A, B]$, this does not say that $X \in \mathrm{SU}(X)$, since one could have $X^{t} \notin \mathrm{USS}\left(X^{t}\right)$ for some $t \notin[A, B]$. But for our purposes we will only need that $X^{m} \in \operatorname{USS}\left(X^{m}\right)$ for $m \in[1,\|\Delta\|]$, hence the proof of Proposition 2.23 allows us to assume this hypothesis.

Remark. Very recently we learnt that in [32], a finite time algorithm to compute the stable super summit set [30] is given. It is possible that similar methods can be used to compute $\mathrm{SU}(X)$ in finite time, but as we said above, we will not need that for our purposes in this paper.

Let us then assume that $X^{m} \in \operatorname{USS}\left(X^{m}\right)$ for $m=1, \ldots,\|\Delta\|$. Recall that we have defined some factors $C_{i}, R_{i}, C_{i}$ and $\boldsymbol{R}_{i}$ related to $X$, for every $i \in \mathbb{Z}$. We can thus define the same elements related to each $X^{m}$, but we need some notation to make the distinction between them, for different values of $m$. The notation $C_{i}\left(X^{m}\right)=$ $\iota\left(\mathbf{c}^{i-1}\left(X^{m}\right)\right)$ would not cause confusion, but it would be too awkward for the formulae below, so we will simplify it by denoting

$$
C_{i}^{(m)}=C_{i}\left(X^{m}\right)=\iota\left(\mathbf{c}^{i-1}\left(X^{m}\right)\right) .
$$


Thus $C_{i}^{(m)}$ has the same definition as $C_{i}$, but related to $X^{m}$ instead of $X$. Later on, we will study the sequence

$$
C_{1}, C_{1}^{(2)}, C_{1}^{(3)}, \ldots
$$

that is,

$$
\iota(X), \iota\left(X^{2}\right), \iota\left(X^{3}\right), \ldots
$$

Notice that, a priori, there does not have to be a relation between them, due to the unexpected increases of supremum that one encounters when taking powers.

Similarly to $C_{i}^{(m)}$, one defines $R_{i}^{(m)}, \boldsymbol{C}_{i}^{(m)}$ and $\boldsymbol{R}_{i}^{(m)}$ in the same way as $R_{i}, \boldsymbol{C}_{i}$ and $\boldsymbol{R}_{i}$, but related to $X^{m}$ instead of $X$. The relation between these elements for different powers of $X$ will be crucial in the sequel.

\section{Rigidity}

3.1. Rigidity of an element and behavior under cyclings and powers. In this section we will define a notion of rigidity for elements in a Garside group, and we will study how rigidity is affected when applying some cyclings or taking some powers. The idea of studying rigidity came from the study of elements whose left normal form changes only in the obvious way under cyclings, decyclings and powers, so their ultra summit sets are easier to study. We call them rigid elements:

Definition 3.1 (Rigid element). Let $X=\Delta^{p} x_{1} \ldots x_{r}$ be in left normal form, with $r>0$. Then $X$ is rigid if the element $\Delta^{p} x_{1} \ldots x_{r} \tau^{-p}\left(x_{1}\right)$ is in left normal form as written.

Notice that if $X$ is rigid, then the cycling of $X$, that is, $\mathbf{c}(X)=\Delta^{p} x_{2} \ldots x_{r} \tau^{-p}\left(x_{1}\right)$ is in left normal form as written. Actually, this latter property is equivalent to $X$ being rigid if $r>1$. But we prefer the definition above, otherwise every element of canonical length 1 would be rigid.

The following are equivalent definitions of rigid elements.

Proposition 3.2. Given $X=\Delta^{p} x_{1} \ldots x_{r} \in G$ with $r>0$, the following conditions are equivalent.

(1) $X$ is rigid.

(2) $\varphi(X) \iota(X)$ is left weighted as written.

(3) $\iota(X) \wedge \iota\left(X^{-1}\right)=1$.

Proof. By definition $X$ is rigid if $\Delta^{p} x_{1} \ldots x_{r} \tau^{-p}\left(x_{1}\right)$ is in left normal form as written. Since $x_{1} \ldots x_{r}$ is already in left normal form, this is equivalent to the left weightedness of $x_{r} \tau^{-p}\left(x_{1}\right)=\varphi(X) \iota(X)$ so conditions 1 and 2 are equivalent. But condition 2 
means $\tau^{-p}\left(x_{1}\right) \wedge \partial\left(x_{r}\right)=1$. We know that $\iota(X)=\tau^{-p}\left(x_{1}\right)$ and also, by Lemma 1.8 , $\iota\left(X^{-1}\right)=\partial\left(x_{r}\right)$. Hence conditions 2 and 3 are also equivalent.

In general, we define the rigidity of an element, in such a way that rigid elements have rigidity 1 .

Definition 3.3. Given $X=\Delta^{p} x_{1} \ldots x_{r}$ in left normal form, with $r>0$, we define the rigidity of $X$ as

$$
\mathcal{R}(X)=k / r
$$

where $k$ is the biggest integer in $\{0, \ldots, r\}$ such that the first $k$ factors in the left normal form of $x_{1} \ldots x_{r} \tau^{-p}\left(x_{1}\right)$ are precisely $x_{1} \ldots x_{k}$. If $r=0$, we define $\mathcal{R}(X)=0$.

The rigidity of an element tells us how many (non- $\Delta$ ) factors of the left normal form of $X$ are preserved when considering $X^{2}$. Notice that $X$ is rigid if and only if $\mathcal{R}(X)=1$.

Example 7. (1) If $X=\Delta \cdot 12 \cdot 21 \cdot 12 \in B_{3}$, then $\mathcal{R}(X)=3 / 3=1$, since $\iota(X)=\tau^{-1}(12)=21$, and $12 \cdot 21 \cdot 12 \cdot 21$ is in left normal form as written. Hence $X$ is rigid.

(2) If $X=13 \cdot 13 \cdot 1 \in B_{4}$, then $\mathcal{R}(X)=2 / 3$, since the left normal form of $(13 \cdot 13 \cdot 1) 13$ is $13 \cdot 13 \cdot 13 \cdot 1$, hence $k=2$ and $r=3$. This means that two thirds of the left normal form of $X$ are preserved when considering $X^{2}=13 \cdot 13 \cdot 13 \cdot 13 \cdot 1 \cdot 1$.

(3) If $X=12132143 \cdot 143 \in B_{5}$ (this is the example at the beginning of Section 2$)$, then $\mathcal{R}(X)=0$, since the left normal form of $(12132143 \cdot 143) \cdot 12132143$ is $\Delta \cdot 2324321 \cdot 14$. Hence, nothing from the left normal form of $X$ is preserved when computing its square $X^{2}=\Delta \cdot 2324321 \cdot 14 \cdot 143$. In this case we say that $X$ has no rigidity, or that it is 0 -rigid. This is, of course, the most difficult case if one tries to relate cyclings and powers of $X$.

Let us see some characterizations of rigidity, and then how rigidity behaves under cyclings or powers of an element.

Lemma 3.4. Let $X \in G$ with $\ell(X)=r>0$ and $\inf (X)=p$. Then $\mathcal{R}(X)=k / r$ if and only if $k$ is the biggest integer such that

$$
\left(X^{2} \Delta^{-2 p}\right) \wedge \Delta^{k}=\left(X \Delta^{-p}\right) \wedge \Delta^{k}
$$

In particular, $\mathcal{R}(X)>0$ if and only if $\inf \left(X^{2}\right)=2 p$ and $\iota\left(X^{2}\right)=\iota(X)$.

Proof. First notice that $k=0$ always satisfies the above condition, because $\left(X^{2} \Delta^{-2 p}\right) \wedge 1=1=\left(X \Delta^{-p}\right) \wedge 1$. Also, no $k>r$ can satisfy the condition, since $\left(X \Delta^{-p}\right)$ only has $r$ factors, and this would imply that $X^{2} \Delta^{-2 p}=X \Delta^{-p}$, 
which is not possible if $r>0$. Hence the biggest integer $k$ satisfying the condition must belong to $\{0, \ldots, r\}$.

The rigidity of $X=\Delta^{p} x_{1} \ldots x_{r}$ is at least $k / r$ if the first $k$ factors in the left normal form of $x_{1} \ldots x_{r} \tau^{-p}\left(x_{1}\right)$ are $x_{1} \ldots x_{k}$. This is the case if and only if the biggest simple prefix of $x_{k} \ldots x_{r} \tau^{-p}\left(x_{1}\right)$ is $x_{k}$, which in turn is the case if and only if the biggest simple prefix of $x_{k} \ldots x_{r} \tau^{-p}\left(x_{1} \ldots x_{r}\right)$ is $x_{k}$. Since $x_{1} \ldots x_{k}$ is in left normal form, the above condition holds if and only if the left normal form of $X^{2}=\Delta^{2 p} \tau^{p}\left(x_{1} \ldots x_{r}\right)\left(x_{1} \ldots x_{r}\right)$ has the form $\Delta^{2 p} \tau^{p}\left(x_{1}\right) \ldots \tau^{p}\left(x_{k}\right) z_{1} \ldots z_{t}$ for some simple elements $z_{1}, \ldots, z_{t}$. This happens if and only if $\left(X^{2} \Delta^{-2 p}\right) \wedge \Delta^{k}=$ $\tau^{-p}\left(x_{1} \ldots x_{k}\right)=\left(X \Delta^{-p}\right) \wedge \Delta^{k}$. This shows that $\mathcal{R}(X)=k / r$ if and only if $k$ is the biggest integer satisfying the latter condition.

Now $\mathcal{R}(X)>0$ if $k$ is at least 1 , where for $k=1$ the above condition reads $X^{2} \Delta^{-2 p} \wedge \Delta=\tau^{-p}\left(x_{1}\right)=\iota(X)$, which is equivalent to $\inf \left(X^{2}\right)=2 p$ and $\iota\left(X^{2}\right)=$ $\iota(X)$. (Notice that if $\inf \left(X^{2}\right)>2 p$ then $X^{2} \Delta^{-2 p} \wedge \Delta=\Delta \neq \iota\left(X^{2}\right)$.)

One can also check the rigidity of an element by looking at its inverse.

Lemma 3.5. Let $X \in G$ with $\ell(X)=r>0$. Then $\mathcal{R}(X)=k / r>0$ if and only if $\sup \left(X^{-2}\right)=2 \sup \left(X^{-1}\right)$ and the final $k$ factors in the left normal forms of $X^{-1}$ and $X^{-2}$ coincide. In particular, $X$ is rigid if and only if $X^{-1}$ is rigid. And also $\mathcal{R}(X)>0$ if and only if $\sup \left(X^{-2}\right)=2 \sup \left(X^{-1}\right)$ and $\varphi\left(X^{-2}\right)=\varphi\left(X^{-1}\right)$.

Proof. The rigid case can be shown independently. We know by Proposition 3.2 that $X$ is rigid if and only if $\iota(X) \wedge \iota\left(X^{-1}\right)=1$, and this condition is invariant under taking inverses. Hence $X$ is rigid if and only if so is $X^{-1}$.

On the other hand, let $\Delta^{p} x_{1} \ldots x_{r}$ be the left normal form of $X$. By Lemma 3.4, $\mathcal{R}(X)=k / r>0$ if and only if the first $k$ factors in the left normal forms of $X^{2} \Delta^{-2 p}$ and $X \Delta^{-p}$ coincide. This means that the left normal form of $X^{2}$ is $\Delta^{q} y_{1} \ldots y_{t}$, where $q=2 p$ and $y_{i}=\tau^{p}\left(x_{i}\right)$ for $i=1, \ldots, k$. By Theorem 1.5, the left normal form of $X^{-1}$ is $\Delta^{-p-r} x_{r}^{\prime} \ldots x_{1}^{\prime}$ where $x_{i}^{\prime}=\tau^{-p-i}\left(\partial\left(x_{i}\right)\right)$, and the left normal form of $X^{-2}$ is $\Delta^{-q-t} y_{t}^{\prime} \ldots y_{1}^{\prime}$, where $y_{i}^{\prime}=\tau^{-q-i}\left(\partial\left(y_{i}\right)\right)$. Then $q=2 p$ means $\sup \left(X^{-2}\right)=$ $2 \sup \left(X^{-1}\right)$, and $y_{i}=\tau^{p}\left(x_{i}\right)$ means $y_{i}^{\prime}=\tau^{-2 p-i}\left(\partial\left(y_{i}\right)\right)=\tau^{-2 p-i}\left(\partial\left(\tau^{p}\left(x_{i}\right)\right)\right)=$ $\tau^{-2 p-i}\left(\tau^{p}\left(\partial\left(x_{i}\right)\right)\right)=\tau^{-p-i}\left(\partial\left(x_{i}\right)\right)=x_{i}^{\prime}$, hence the result follows.

In the case of nonzero rigidity, we will be able to state some common property of all powers of $X$.

Corollary 3.6. Let $X \in G$ with $\ell(X)>0$. If $\mathcal{R}(X)>0$, then $\iota(X)=\iota\left(X^{m}\right)$ (whence $\left.\varphi\left(X^{-1}\right)=\varphi\left(X^{-m}\right)\right)$, and also $\inf \left(X^{m}\right)=m \inf (X)\left(\right.$ whence $\sup \left(X^{-m}\right)=$ $\left.m \sup \left(X^{-1}\right)\right)$ for every $m \geq 1$.

Proof. We show the equalities $\iota(X)=\iota\left(X^{m}\right)$ and $\inf \left(X^{m}\right)=m \inf (X)$. By Corollary 1.6, these imply $\varphi\left(X^{-1}\right)=\partial^{-1}(\iota(X))=\partial^{-1}\left(\iota\left(X^{m}\right)\right)=\varphi\left(X^{-m}\right)$ and $\sup \left(X^{-m}\right)=-\inf \left(X^{m}\right)=-m \inf (X)=m \sup \left(X^{-1}\right)$. 
The result is trivially true for $m=1$. Let $\Delta^{p} x_{1} \ldots x_{r}$ be the left normal form of $X$, and suppose that $\iota\left(X^{m}\right)=\iota(X)=\tau^{-p}\left(x_{1}\right)$ and $\inf \left(X^{m}\right)=m \inf (X)=m p$ for some $m \geq 1$. Write then $X^{m}=\Delta^{m p} \tau^{m p-p}\left(x_{1}\right) y_{2} \ldots y_{s}$ in left normal form.

Observe that $\Delta \nprec x_{1} \ldots x_{r} \tau^{-p}\left(x_{1}\right)$ because $\mathcal{R}(X)>0$. Hence, there is no unexpected appearance of $\Delta$ in the product $X X^{m}$, which in turn implies $\inf \left(X^{m+1}\right)=$ $\inf (X)+\inf \left(X^{m}\right)=p+m p=(m+1) \inf (X)$. Furthermore, $\iota\left(X^{m+1}\right)=$ $\iota\left(\Delta^{p} x_{1} \ldots x_{r} \tau^{-p}\left(x_{1}\right) \tau^{-m p}\left(y_{2} \ldots y_{s}\right) \Delta^{m p}\right)=\iota\left(\Delta^{p} x_{1} \ldots x_{r} \tau^{-p}\left(x_{1}\right)\right)=\tau^{-p}\left(x_{1}\right)=$ $\iota(X)$, and the result follows.

Let us see that rigidity cannot decrease by cyclings.

Proposition 3.7. Let $X \in \operatorname{SSS}(X)$ with $\ell(X)>0$. Then $\mathcal{R}(X) \leq \mathcal{R}\left(\mathbf{c}^{t}(X)\right)$ for all $t \geq 1$. Furthermore, if $X \in \operatorname{USS}(X)$, equality holds.

Proof. Let $p=\inf (X)$. By definition of rigidity, $\mathcal{R}(X)=k / r$ means that $k$ is the biggest integer such that $X \iota(X) \wedge \Delta^{p+k}=X \wedge \Delta^{p+k}$. If we apply the transport map defined in [24] to this equality, we get

$$
\mathbf{c}(X) \iota(\mathbf{c}(X)) \wedge \Delta^{p+k}=\mathbf{c}(X) \wedge \Delta^{p+k},
$$

which means that $\mathcal{R}(\mathbf{c}(X)) \geq k / r$ (notice that $\ell(\mathbf{c}(X))=r$ since $X \in \operatorname{SSS}(X)$ ). Applying the same reasoning to every cycling of $X$, one has $\mathcal{R}\left(\mathbf{c}^{t-1}(X)\right) \leq \mathcal{R}\left(\mathbf{c}^{t}(X)\right)$ for every $t \geq 1$, so the result follows.

If $X \in \operatorname{USS}(X)$, one cannot have $\mathcal{R}(X)<\mathcal{R}\left(\mathbf{c}^{t}(X)\right)$ for some $t$, since some further cycling of $\mathbf{c}^{t}(X)$ would be equal to $X$, yielding the contradiction $\mathcal{R}(X)<$ $\mathcal{R}(X)$.

If an element has some rigidity, its conjugating elements for cycling $C_{i}$ satisfy the following useful property.

Lemma 3.8. Let $X \in \operatorname{SSS}(X)$ with $\ell(X)>0$. If $\mathcal{R}(X)>0$, the left normal form of $C_{m}$ is precisely $C_{1} C_{2} \ldots C_{m}$ for every $m \geq 1$.

Proof. Let $\Delta^{p} x_{1} \ldots x_{r}$ be the left normal form of $X$, and let $\Delta^{p} y_{1} \ldots y_{r}$ be the left normal form of $\mathbf{c}(X)=\Delta^{p} x_{2} \ldots x_{r} \tau^{-p}\left(x_{1}\right)$. Since $\mathcal{R}(X)>0$, the left normal form of $x_{1} x_{2} \ldots x_{r} \tau^{-p}\left(x_{1}\right)$ is $x_{1} y_{1} \ldots y_{r}$. Since $C_{1}=\tau^{-p}\left(x_{1}\right), C_{2}=\tau^{-p}\left(y_{1}\right)$ and $x_{1} y_{1}$ is left weighted, it follows that $C_{1} C_{2}$ is left weighted. Applying the same reasoning to $\mathrm{c}^{i-1}(X)$ for every $i \geq 2$, it follows that $C_{i} C_{i+1}$ is left weighted as written, hence $C_{1} \ldots C_{m}$ is the left normal form of $C_{m}$.

It is easy to see that if an element $X$ is rigid, then every power of $X$ is rigid. We can generalize this to every element $X \in \operatorname{USS}(X)$, showing that the rigidity of $X$ can never decrease by taking powers. 
Proposition 3.9. Let $X \in \operatorname{USS}(X)$ with $\ell(X) \geq 1$. Then $\mathcal{R}(X) \leq \mathcal{R}\left(X^{m}\right)$ for every $m>1$.

Proof. Let $\ell(X)=r$ and $\mathcal{R}(X)=k / r$. If $k=0$ the result is trivial, so we can assume that $k>0$. In the case $r=1$ this would mean that $X$ is rigid, hence every power of $X$ is rigid and the result would also be true. Therefore we will also assume that $\ell(X)=r>1$. Let $\Delta^{p} x_{1} \ldots x_{r}$ be the left normal form of $X$. Recall that by Corollary $3.6 \inf \left(X^{t}\right)=t p$ for every $t \geq 1$. We will show that for $m \geq 1$ one has

$$
\left(X^{m} \Delta^{-p m}\right) \wedge \Delta^{m k}=C_{m k} .
$$

Recall from Theorem 2.9 that $\left(X^{m k} \Delta^{-p m k}\right) \wedge \Delta^{m k}=C_{m k}$. Since one has $X^{m} \Delta^{-p m} \preccurlyeq X^{m k} \Delta^{-p m k}$ for every $k>0$, (notice that the infimum of both elements is 0 ) it follows that

$$
\left(X^{m} \Delta^{-p m}\right) \wedge \Delta^{m k} \preccurlyeq\left(X^{m k} \Delta^{-p m k}\right) \wedge \Delta^{m k}=C_{m k},
$$

hence we only need to show that $C_{m k} \preccurlyeq X^{m} \Delta^{-p m}$.

We will first show that $C_{i}=\tau^{-p}\left(x_{i}\right)$ for $i=1, \ldots, k$. This will be done by proving that, for $i=0, \ldots, k-1$ the first $k-i$ non- $\Delta$ factors in the left normal form of $\mathbf{c}^{i}(X)$ are $x_{i+1} \ldots x_{k}$. Indeed, this is trivially true for $i=0$. If we assume the claim true for some $i, 0 \leq i<k-1$, we have $\mathbf{c}^{i}(X)=\Delta^{p} x_{i+1} \ldots x_{k} y_{k+1} \ldots y_{r+i}$. By Proposition 3.7 we know that $\mathcal{R}\left(\mathbf{c}^{i}(X)\right) \geq k / r$, hence the first $k-1$ non- $\Delta$ factors in the left normal form of $\mathbf{c}^{i+1}(X)=\Delta^{p} x_{i+2} \ldots x_{k} y_{k+1} \ldots y_{r+i} \tau^{-p}\left(x_{i+1}\right)$ are precisely $x_{i+2} \ldots x_{k} y_{k+1} \ldots y_{k+i}$. In particular, the first $k-(i+1)$ non- $\Delta$ factors are $x_{i+2} \ldots x_{k}$, thus the claim is shown. This implies that

$$
C_{k}=C_{1} \ldots C_{k}=\tau^{-p}\left(x_{1} \ldots x_{k}\right),
$$

hence $\boldsymbol{C}_{k} \preccurlyeq X \Delta^{-p}$.

Now suppose that $\boldsymbol{C}_{m k} \preccurlyeq X^{m} \Delta^{-p m}$ for some $m \geq 1$. If we apply $k$ times the transport defined in [24], which preserves $\preccurlyeq$, we obtain

$$
\boldsymbol{C}_{[k, m k]} \preccurlyeq\left(\mathbf{c}^{k}(X)\right)^{m} \Delta^{-p m} .
$$

As $C_{k}=\tau^{-p}\left(x_{1} \ldots x_{k}\right)$, this implies $\mathbf{c}^{k}(X)=X^{C_{k}}=\Delta^{p} x_{k+1} \ldots x_{r} \tau^{-p}\left(x_{1} \ldots x_{k}\right)$. Hence,

$$
\begin{aligned}
X^{m+1} & =\left(\Delta^{p} x_{1} \ldots x_{r}\right)^{m+1} \\
& =\Delta^{p} x_{1} \ldots x_{k}\left(x_{k+1} \ldots x_{r} \Delta^{p} x_{1} \ldots x_{k}\right)^{m} x_{k+1} \ldots x_{r} \\
& =\Delta^{p} x_{1} \ldots x_{k}\left(\Delta^{p} \tau^{p}\left(x_{k+1} \ldots x_{r}\right) x_{1} \ldots x_{k}\right)^{m} x_{k+1} \ldots x_{r} \\
& =\tau^{-p}\left(x_{1} \ldots x_{k}\right)\left(\mathbf{c}^{k}(X)\right)^{m} \tau^{-p}\left(x_{k+1} \ldots x_{r}\right) \Delta^{p} .
\end{aligned}
$$


Since $\tau^{-p}\left(x_{1} \ldots x_{k}\right)=\boldsymbol{C}_{k}$, and $\boldsymbol{C}_{[k, m k]} \preccurlyeq\left(\mathbf{c}^{k}(X)\right)^{m} \Delta^{-p m}$, it follows that

$$
\boldsymbol{C}_{(m+1) k}=\boldsymbol{C}_{k} \boldsymbol{C}_{[k, m k]} \preccurlyeq X^{m+1} \Delta^{-(m+1) p},
$$

as we wanted to show. Hence $\left(X^{m} \Delta^{-p m}\right) \wedge \Delta^{m k}=C_{m k}$ for every $m \geq 1$.

Now recall from Lemma 3.8 that, since $k>0$, the left normal form of $\boldsymbol{C}_{m k}$ is $C_{1} \ldots C_{m k}$. Hence, for every $m \geq 1$ one has

$$
\left(X^{2 m} \Delta^{-2 p m}\right) \wedge \Delta^{m k}=C_{2 m k} \wedge \Delta^{m k}=C_{m k}=\left(X^{m} \Delta^{-p m}\right) \wedge \Delta^{m k} .
$$

By Lemma 3.4, and since $\ell\left(X^{m}\right) \leq m r$, this implies that $\mathcal{R}\left(X^{m}\right) \geq \frac{m k}{m r}=\frac{k}{r}=$ $\mathcal{R}(X)$, as we wanted to show.

Remark. The main difference between rigidity of cyclings and rigidity of powers is that, while iterated cycling of $X \in \operatorname{SSS}(X)$ yields a non-decreasing sequence

$$
\mathcal{R}(X) \leq \mathcal{R}(\mathbf{c}(X)) \leq \mathcal{R}\left(\mathbf{c}^{2}(X)\right) \leq \cdots,
$$

this does not happen for powers of $X$, even if $X \in \operatorname{USS}(X)$. For instance, if $X=12132143 \cdot 143 \in B_{5}$ is the example at the beginning of Section 2, one has

$$
\begin{array}{rll}
\mathcal{R}(X)=0, & \mathcal{R}\left(X^{2}\right)=0, & \mathcal{R}\left(X^{3}\right)=1, \\
\mathcal{R}\left(X^{4}\right)=0, & \mathcal{R}\left(X^{5}\right)=0, & \mathcal{R}\left(X^{6}\right)=1, \ldots
\end{array}
$$

Notice that this is not in contradiction with Proposition 3.9, where the rigidity of $X^{m}$ is compared with that of $X$, not with the rigidity of the intermediate powers.

The above results imply that elements having some rigidity behave nicely with respect to powers and cyclings, in the following sense:

Corollary 3.10. If $X \in \operatorname{USS}(X)$ with $\ell(X) \geq 1$ and $\mathcal{R}(X)>0$, then one has $\left(\mathbf{c}^{t}(X)\right)^{m}=\mathbf{c}^{t}\left(X^{m}\right)$ for every $t, m \geq 1$.

Proof. By Lemma 3.8, the left normal form of $C_{m}$ is $C_{1} \ldots C_{m}$. If $\ell(X)>1$ this implies, by Theorem 2.9, that $C_{1}=\iota\left(C_{1} \ldots C_{m}\right)=\iota\left(X^{m}\right)$. If $\ell(X)=1$ then $X^{m}=C_{1} \ldots C_{m} \Delta^{m p}$ where $p=\inf (X)$, so also in this case we have $\iota\left(X^{m}\right)=C_{1}$. Hence $\mathbf{c}\left(X^{m}\right)=\left(X^{m}\right)^{C_{1}}=\left(X^{C_{1}}\right)^{m}=(\mathbf{c}(X))^{m}$, and the result is true for $t=1$. If the result is true for some $t-1$, it suffices to apply the previous case to $\mathbf{c}^{t-1}(X)$, which has some rigidity by Proposition 3.7, to obtain

$$
\mathbf{c}^{t}\left(X^{m}\right)=\mathbf{c}\left(\mathbf{c}^{t-1}\left(X^{m}\right)\right)=\mathbf{c}\left(\left(\mathbf{c}^{t-1}(X)\right)^{m}\right)=\left(\mathbf{c}\left(\mathbf{c}^{t-1}(X)\right)\right)^{m}=\left(\mathbf{c}^{t}(X)\right)^{m} .
$$

Corollary 3.11. Let $X \in \operatorname{USS}(X)$ with $\ell(X) \geq 1$. If $\mathcal{R}(X)>0$ then $X^{m}$ belongs to a closed orbit under cycling, for every $m \geq 1$. 
Proof. Let $N$ be the orbit length of $X$. By Corollary 3.10, $\mathbf{c}^{N}\left(X^{m}\right)=\left(\mathbf{c}^{N}(X)\right)^{m}=$ $X^{m}$, so the result follows.

Remark. The above result does not imply that $X^{m} \in \operatorname{USS}(X)$, since it could happen that $X^{m} \notin \operatorname{SSS}\left(X^{m}\right)$. But the fact that $X^{m}$ belongs to a closed orbit under cycling will be enough for our purposes.

3.2. The ultra summit set of a rigid element is made of rigid elements. By the above discussion on rigidity, we know that if $X \in \operatorname{USS}(X)$ is rigid, then the whole orbit of $X$ under cycling is made of rigid elements. But what about the other orbits in $\operatorname{USS}(X)$ ? In this subsection we will show that, if $\ell(X)>1$, all orbits in $\operatorname{USS}(X)$ are made of rigid elements. Hence $\operatorname{USS}(X)$ is just the set of rigid conjugates of $X$. We start with three small results.

Lemma 3.12. Given $X \in \operatorname{USS}(X)$ then $\mathbf{d}(X) \in \operatorname{USS}(X)$.

Proof. Let $\Delta^{p} x_{1} \ldots x_{r}$ be the left normal form of $X$. Notice that $X^{X}=X \in$ $\operatorname{USS}(X)$ and that $X^{\Delta^{p+r-1}}=\tau^{p+r-1}(X) \in \operatorname{USS}(X)$. Then, by Theorem 1.16, $\mathbf{d}(X)=X^{\left(\Delta^{p} x_{1} \ldots x_{r-1}\right)}=X^{X \wedge \Delta^{p+r-1}} \in \operatorname{USS}(X)$.

Lemma 3.13. If $X \in G$ is rigid, then $\mathbf{c}^{i}(X)$ and $\mathbf{d}^{i}(X)$ are rigid for every $i \geq 1$. Moreover $X \in \operatorname{USS}(X)$, and if $e \geq 1$ is such that $\Delta^{e}$ is central, then $\mathbf{c}^{m}(X)=X$ for some $m \leq \ell(X) e$.

Proof. Let $\Delta^{p} x_{1} \ldots x_{r}$ be the left normal form of $X$. By Proposition 3.7, all iterated cyclings of $X$ are rigid. Then one can easily show by recurrence that if $i=k r+j$ with $0 \leq j \leq r-1$, the left normal form of $\mathbf{c}^{i}(X)$ is equal to $\Delta^{p} \tau^{k p}\left(x_{j+1}\right) \ldots \tau^{k p}\left(x_{r}\right) \tau^{(k+1) p}\left(x_{1}\right) \ldots \tau^{(k+1) p}\left(x_{j}\right)$. Hence, if $e$ is such that $\tau^{e}=1$, one has $\mathbf{c}^{e r}(X)=\Delta^{p} \tau^{e p}\left(x_{1}\right) \ldots \tau^{e p}\left(x_{r}\right)=\Delta^{p} x_{1} \ldots x_{r}=X$, so $X$ belongs to a closed orbit under cycling, and the orbit length is a divisor of $r e=\ell(X) e$.

By Lemma 3.5 $X$ is rigid if and only if so is $X^{-1}$. This means that $\mathbf{c}^{i}\left(X^{-1}\right)$ is rigid for every $i \geq 1$. But we know by [20] that $\tau^{-i}\left(\mathbf{c}^{i}\left(X^{-1}\right)\right)=\left(\mathbf{d}^{i}(X)\right)^{-1}$, hence $\mathbf{d}^{i}(X)$ is also rigid for every $i \geq 1$.

Furthermore, by the above arguments $X^{-1}$ belongs to a closed orbit under cycling, thus $X$ belongs to a closed orbit under decycling. But an element belonging to closed orbits under cycling and decycling belongs to its ultra summit set (since one can always reach the ultra summit set by iterated cycling and decycling), so it follows that $X \in \operatorname{USS}(X)$.

For elements which belong to their ultra summit set, the converse of Lemma 3.13 is also true. 
Lemma 3.14. If $Y \in \mathrm{USS}(X)$ is not rigid, then neither $\mathbf{c}^{i}(Y)$ nor $\mathbf{d}^{i}(Y)$ are rigid for any $i \geq 1$.

Proof. It clearly suffices to show the result for $i=1$. First, $\mathbf{c}(Y)$ has the same rigidity as $Y$ by Proposition 3.7, hence it cannot be rigid.

Now suppose that $\mathbf{d}(Y)$ is rigid. This is clearly not possible if $\ell(Y)=1$, so we can suppose that $\ell(Y)>1$. If $\Delta^{p} y_{1} \ldots y_{r}$ is the left normal form of $Y$, then $\mathbf{d}(Y)=\Delta^{p} \tau^{p}\left(y_{r}\right) y_{1} \ldots y_{r-1}$, although this decomposition is not the left normal form of $\mathbf{d}(Y)$. Nevertheless, since $y_{1} \ldots y_{r-1}$ is in left normal form, we know, by the left normal form algorithm (see for instance [21]) that there is a decomposition $y_{i}=a_{i} b_{i}$ for $i=1, \ldots, r-1$ such that the left normal form of $\mathbf{d}(Y)$ is precisely $\Delta^{p}\left(\tau^{p}\left(y_{r}\right) a_{1}\right)\left(b_{1} a_{2}\right) \ldots\left(b_{r-2} a_{r-1}\right)\left(b_{r-1}\right)$.

Since we are assuming that $\mathbf{d}(Y)$ is rigid, we have that $\left(b_{r-1}\right)\left(y_{r} \tau^{-p}\left(a_{1}\right)\right)$ is left weighted as written. Notice that this implies that $y_{r-1}\left(y_{r} \tau^{-p}\left(a_{1}\right)\right)$ is left weighted as written. Therefore the left normal form of $y_{1} \ldots y_{r} \tau^{-p}\left(y_{1}\right)$ is precisely $y_{1} \ldots y_{r-1}\left(y_{r} \tau^{-p}\left(a_{1}\right)\right) \tau^{-p}\left(b_{1}\right)$. In other words, $\mathcal{R}(Y)=\frac{r-1}{r}$. By Proposition 3.7, all iterated cyclings of $Y$ have rigidity $\frac{r-1}{r}$. This implies, in particular, that

$$
\mathbf{c}^{r-1}(Y)=Y^{\tau^{-p}\left(y_{1} \ldots y_{r-1}\right)}=\Delta^{p} y_{r} \tau^{-p}\left(y_{1} \ldots y_{r-1}\right)=\tau^{-p}(\mathbf{d}(Y)),
$$

but this latter element is supposed to be rigid. A contradiction. Hence no iterated decycling of $Y$ can be rigid.

We can finally prove the main result concerning the elements of the ultra summit set of a rigid element.

Theorem 3.15. Let $X$ be rigid and $\ell(X)>1$. Then every element in $\operatorname{USS}(X)$ is rigid.

Proof. Suppose that there exists an element in $\operatorname{USS}(X)$ which is not rigid. Since every two elements in $\operatorname{USS}(X)$ are connected by a sequence of conjugations by simple elements, there must be a non-rigid element in $\operatorname{USS}(X)$ which is the conjugate of a rigid one by a simple element. Hence we can assume without loss of generality that $X^{s}=Y$ for some non-rigid element $Y \in \operatorname{USS}(X)$ and some simple element $s$. We will also assume that $s$ is a maximal element (with respect to $\preccurlyeq$ ) in the set of all simple elements conjugating $X$ to a non-rigid element in $\operatorname{USS}(X)$. We will get a contradiction by showing that $X$ is conjugate to $\mathbf{c}(\mathbf{d}(Y))$ by a simple element which is a proper right multiple of $s$.

Let $\Delta^{p} x_{1} \ldots x_{r}$ be the left normal form of $X$ and let $\Delta^{p} y_{1} \ldots y_{r}$ be the left normal form of $Y$. Since $X^{s}=Y$, it is known by [24] that there exist simple elements $s_{0}, \ldots, s_{r}$ such that $s_{0}=\tau^{p}(s), s_{r}=s$ and $y_{i}=s_{i-1}^{-1} x_{i} s_{i}$ for $i=1, \ldots, r$. That is, the left normal form of $Y$ is $\Delta^{p} y_{1} \ldots y_{r}=\Delta^{p}\left(s_{0}^{-1} x_{1} s_{1}\right)\left(s_{1}^{-1} x_{2} s_{2}\right) \ldots\left(s_{r-1}^{-1} x_{r} s_{r}\right)$. 
Now consider $\mathbf{d}(X)$ and $\mathbf{d}(Y)$. By Lemma 3.12, these two elements belong to $\operatorname{USS}(X)$. Since $X$ is rigid, the left normal form of $\mathbf{d}(X)$ is $\Delta^{p} \tau^{p}\left(x_{r}\right) x_{1} \ldots x_{r-1}$. However, $Y$ is not rigid, so the left normal form of $\mathbf{d}(Y)$ is not $\Delta^{p} \tau^{p}\left(y_{r}\right) y_{1} \ldots y_{r-1}$, since $\tau^{p}\left(y_{r}\right) y_{1}$ is not left weighted as written. (Here we use the fact that $r=\ell(X)>1$.) Hence, $\iota(\mathbf{d}(Y))=y_{r} t$ for some nontrivial simple element $t$.

The elements $\mathbf{d}(X)$ and $\mathbf{d}(Y)$ are also connected through a conjugation by a simple element. Namely, $\mathbf{d}(X)^{s_{r-1}}=\Delta^{p} \tau^{p}\left(s_{r-1}\right)^{-1} \tau^{p}\left(x_{r}\right) x_{1} \ldots x_{r-1} s_{r-1}=$ $\Delta^{p} \tau^{p}\left(y_{r}\right) y_{1} \ldots y_{r-1}=\mathbf{d}(Y)$. Hence, by [24] again, there exist simple elements $t_{0}, \ldots, t_{r}$ such that $t_{0}=\tau^{p}\left(s_{r-1}\right), t_{r}=s_{r-1}$ and the left normal form of $\mathbf{d}(Y)$ is $\Delta^{p}\left(t_{0}^{-1} \tau^{p}\left(x_{r}\right) t_{1}\right)\left(t_{1}^{-1} x_{1} t_{2}\right) \ldots\left(t_{r-1}^{-1} x_{r-1} t_{r}\right)$. So $\iota(\mathbf{d}(Y))=\tau^{-p}\left(t_{0}\right)^{-1} x_{r} \tau^{-p}\left(t_{1}\right)=$ $s_{r-1}^{-1} x_{r} \tau^{-p}\left(t_{1}\right)$. Since we saw that $\iota(\mathbf{d}(Y))=y_{r} t=\left(s_{r-1}^{-1} x_{r} s\right) t$, it follows that $s t=\tau^{-p}\left(t_{1}\right)$, which is a simple element. If we denote $u=\tau^{-p}\left(t_{1}\right)$, we just showed that $s \prec u$ (strict) and that $\iota(\mathbf{d}(Y))=s_{r-1}^{-1} x_{r} u$.

Finally, we observe that $X^{u}=\left(\left(X^{x_{r}^{-1}}\right)^{s_{r-1}}\right)^{s_{r-1}^{-1} x_{r} u}=\left(\mathbf{d}(X)^{s_{r-1}}\right)^{s_{r-1}^{-1} x_{r} u}=$ $\mathbf{d}(Y)^{\iota(\mathbf{d}(Y))}=\mathbf{c}(\mathbf{d}(Y))$. But since $Y$ is not rigid and belongs to USS $(X)$, Lemma 3.14 tells us that $\mathbf{d}(Y)$ is not rigid. Since $\mathbf{d}(Y)$ also belongs to $\operatorname{USS}(X)$ by Lemma 3.12, it follows again by Lemma 3.14 that $\mathbf{c}(\mathbf{d}(Y))$ is not rigid, and belongs to $\operatorname{USS}(X)$. But $s \supsetneqq u$, so this contradicts the maximality of $s$, and we are done.

Corollary 3.16. If $X$ is rigid and $\ell(X)>1$, then $\operatorname{USS}(X)$ is the set of rigid conjugates of $X$.

Proof. Let $Y$ be a rigid conjugate of $X$. Since $Y$ is rigid, it belongs to its ultra summit set and since it is conjugate to $X$, its ultra summit set is precisely $\operatorname{USS}(X)$. Conversely, every element in $\operatorname{USS}(X)$ is rigid by the above result.

Corollary 3.17. If $X$ is rigid and $\ell(X)>1$, then $\operatorname{USS}\left(X^{-1}\right)$ is the set of inverses of the elements in $\operatorname{USS}(X)$.

Proof. This is a direct consequence of Corollary 3.16 and the fact that $Y \in G$ is rigid if and only if $Y^{-1}$ is rigid (Lemma 3.5).

Remark. If $\ell(X)=1$, then $\operatorname{USS}(X)$ may contain rigid and non-rigid elements. For instance, the simple element $12321435 \in B_{6}$ is rigid (since $12321435 \cdot 12321435$ is left weighted), but it is conjugate (by 23) to the simple element 12134325, which is not rigid (the left normal form of 1213432512134325 is $1213432514 \cdot 213245$ ). Clearly both elements belong to the ultra summit set, since they are simple.

3.3. Elements having a rigid power. In this section we will characterize elements $X \in G$ having a rigid power $X^{m}$ for some integer $m \neq 0$. Notice that such an element cannot be periodic. Otherwise, since rigidity is preserved by powers, some rigid power of $X$ (which, by definition, has positive canonical length) would also be a 
power of $\Delta$ (which has zero canonical length), and this is not possible. If the element $X$ belongs to its ultra summit set, we can say something more.

Proposition 3.18. Let $X \in \operatorname{USS}(X)$ with $\ell(X) \geq 1$. If $X$ has a rigid power, then there exists some $M>0$ such that $\boldsymbol{C}_{M}=\Delta^{k} X^{t}$ for some integers $k, t$, where $t>0$ and $\Delta^{k}$ is central.

Proof. Let $p=\inf (X)$. If $\ell(X)=1$ then $X^{M}=C_{M} \Delta^{p M}$ for every $M$, so we just need to take $M$ big enough so that $\Delta^{M}$ is central, and we are done. Hence we can assume that $\ell(X)>1$.

Let $e>0$ be such that $\Delta^{e}$ is central, let $m>0$ be such that $X^{m}$ is rigid, and let $N$ be the orbit length of $X$ under cycling. Consider $T=e m N$. By Lemma 2.4 one has $X^{T}=\boldsymbol{C}_{T} \boldsymbol{R}_{T} \Delta^{p T}$, and by Proposition 2.8, $\varphi\left(\boldsymbol{C}_{T}\right) \iota\left(\boldsymbol{R}_{T}\right)$ is left weighted. Since $T$ is a multiple of $e, \Delta^{p T}$ is central. Since $T$ is a multiple of $m$, it follows that $X^{T}$ is rigid, hence $\varphi\left(X^{T}\right) \iota\left(X^{T}\right)=\varphi\left(\boldsymbol{R}_{T}\right) \iota\left(\boldsymbol{C}_{T}\right)$ is also left weighted (notice that the equality holds since $\Delta^{p T}$ is central). Finally, since $T$ is a multiple of $N$, it follows that $\boldsymbol{C}_{T}$ commutes with $X$, thus it commutes with $X^{T}$. This implies

$$
X^{T}=\left(X^{T}\right)^{\boldsymbol{C}_{T}}=\boldsymbol{R}_{T} \Delta^{p T} \boldsymbol{C}_{T}=\Delta^{p T} \boldsymbol{R}_{T} \boldsymbol{C}_{T} .
$$

Moreover, since $\varphi\left(\boldsymbol{R}_{T}\right) \iota\left(\boldsymbol{C}_{T}\right)$ is left weighted, one has $\varphi\left(X^{T}\right)=\varphi\left(\boldsymbol{C}_{T}\right)$. Hence $\varphi\left(\boldsymbol{C}_{T}\right) \iota\left(\boldsymbol{C}_{T}\right)=\varphi\left(X^{T}\right) \iota\left(X^{T}\right)$ is left weighted by the rigidity of $X^{T}$, so it follows that $\boldsymbol{C}_{T}$ is also rigid. In particular, $\inf \left(\left(\boldsymbol{C}_{T}\right)^{k}\right)=k \inf \left(\boldsymbol{C}_{T}\right)$ for every $k>0$. Since $T$ is a multiple of $N$, one has $\left(\boldsymbol{C}_{T}\right)^{k}=\boldsymbol{C}_{T k}$. Hence, by considering a suitable multiple of $T$, we can assume that $\inf \left(\boldsymbol{C}_{T}\right)$ is a multiple of $e$, that is, $\boldsymbol{C}_{T}=\Delta^{e q} y_{1} \ldots y_{r}$, and $\boldsymbol{R}_{T}=z_{1} \ldots z_{s}$, where $y_{r} z_{1}$ and $z_{s} y_{1}$ are left weighted. Then one has

$$
\Delta^{e(p m N+q)} y_{1} \ldots y_{r} z_{1} \ldots z_{s}=X^{T}=\Delta^{e(p m N+q)} z_{1} \ldots z_{s} y_{1} \ldots y_{r},
$$

where both decompositions of $X^{T}$ are in left normal form. In other words, the left normal form of $X^{T}$ is invariant under some cyclic permutations of its factors. This is only possible if there is some rigid element $Y=y_{1} \ldots y_{a}($ where $a=\operatorname{gcd}(r, s))$, such that $Y^{i}=y_{1} \ldots y_{r}$ and $Y^{j}=y_{1} \ldots y_{r} z_{1} \ldots z_{s}$ for some $i, j>0$. But then $\mathbf{C}_{T j}=\left(\mathbf{C}_{T}\right)^{j}=\left(\Delta^{e q} y_{1} \ldots y_{r}\right)^{j}=\Delta^{e q j} Y^{i j}$. Since $Y^{i j}=\left(y_{1} \ldots y_{r} z_{1} \ldots z_{s}\right)^{i}$, it follows that $Y^{i j}=\Delta^{e k^{\prime}} X^{T i}$ for some $k^{\prime} \in \mathbb{Z}$. Denoting $M=T j, t=T i$ and $k=e\left(k^{\prime}+q j\right)$, one finally obtains $C_{M}=\Delta^{k} X^{t}$, as we wanted to show.

We will now show that the converse of Proposition 3.18 is also true for elements of canonical length greater than 1 , by the following two results.

Proposition 3.19. Let $X \in \operatorname{USS}(X)$ with $\ell(X)>1$. Suppose that $\boldsymbol{C}_{M}=\Delta^{k} X^{t}$ for some integers $M, k, t$, where $M, t>0$ and $\Delta^{k}$ is central. Then $\mathcal{R}\left(X^{-m}\right)>0$ for some $m>0$. 
Proof. Notice that $C_{M}$ commutes with $X$, hence $M$ is a multiple of the orbit length of $X$, and then $\left(C_{M}\right)^{r}=C_{M r}$ for every $r \geq 1$. This implies $C_{2 M}=\left(C_{M}\right)^{2}=$ $\Delta^{2 k}\left(X^{t}\right)^{2}$, where $\Delta^{2 k}$ is also central. Hence, replacing $M$ by a multiple if necessary, we can assume that $M \geq\|\Delta\|$.

By Lemma 2.4, $\sup \left(\boldsymbol{C}_{M}\right)=M$ and $\sup \left(\boldsymbol{C}_{2 M}\right)=2 M$. Hence $\sup \left(\left(X^{t}\right)^{2}\right)=$ $-2 k+2 M=2(-k+M)=2 \sup \left(X^{t}\right)$. At the same time, since $M$ is a multiple of the orbit length of $X$ and $M \geq\|\Delta\|$, one has $\varphi\left(C_{M}\right)=F\left(\mathbf{c}^{M}(X)\right)=F(X)$ and also $\varphi\left(\boldsymbol{C}_{2 M}\right)=F(X)$. Therefore $\varphi\left(\left(X^{t}\right)^{2}\right)=\varphi\left(\boldsymbol{C}_{2 M}\right)=F(X)=\varphi\left(\boldsymbol{C}_{M}\right)=\varphi\left(X^{t}\right)$. By Lemma 3.5, this means that $\mathcal{R}\left(X^{-t}\right)>0$, so we take $m=t$ and we are done.

Proposition 3.20. Let $X \in \operatorname{USS}(X)$ with $\ell(X)>1$. Suppose that $C_{M}=\Delta^{k} X^{t}$ for some integers $M, k, t$, where $M, t>0$ and $\Delta^{k}$ is central. Then $X^{T}$ is rigidfor some $T>0$.

Proof. We know by Proposition 3.19 that $\mathcal{R}\left(X^{-m}\right)>0$ for some $m>0$. We also know that $C_{M r}=\Delta^{k r} X^{t r}$ for every $r \geq 1$. Hence, replacing $M$ by a multiple, if necessary, we can assume that both $M$ and $t$ are multiples of $m$. Since $\mathcal{R}\left(X^{-m}\right)>0$, and $M$ and $t$ are multiples of $m$, Corollary 3.6 implies that $\varphi\left(X^{M}\right)=\varphi\left(X^{m}\right)=$ $\varphi\left(X^{t}\right)=\varphi\left(\boldsymbol{C}_{M}\right)$. Notice that $M-t \neq 0$, otherwise $\boldsymbol{R}_{M}$ would be a power of $\Delta$, while $\ell\left(\boldsymbol{R}_{M}\right)>0$ by Lemma 2.4. Hence $|M-t|$ is also a nontrivial multiple of $m$, so we have $\varphi\left(\boldsymbol{C}_{\boldsymbol{M}}\right)=\varphi\left(X^{|\boldsymbol{M}-t|}\right)$.

On the other hand, by Lemma 2.4 we get $X^{M}=C_{M} \boldsymbol{R}_{M} \Delta^{p M}=\Delta^{k} X^{t} \boldsymbol{R}_{M} \Delta^{p M}$, where $p=\inf (X)$. Since $\Delta^{k}$ is central, this means $\boldsymbol{R}_{M}=X^{-t} \Delta^{-k} X^{M} \Delta^{-p M}=$ $X^{M-t} \Delta^{-k-M p}$. Recall that $\ell\left(\boldsymbol{R}_{M}\right)>0$, hence $\iota\left(\boldsymbol{R}_{M}\right)=\iota\left(X^{M-t}\right)$. Moreover $M-t>0$, otherwise we would have $\varphi\left(\boldsymbol{C}_{\boldsymbol{M}}\right)=\varphi\left(X^{t-M}\right)$ and $\iota\left(\boldsymbol{R}_{\boldsymbol{M}}\right)=\iota\left(X^{M-t}\right)=$ $\partial\left(\varphi\left(X^{t-M}\right)\right)$, whence $\varphi\left(\boldsymbol{C}_{M}\right) \iota\left(\boldsymbol{R}_{M}\right)=\Delta$ and this contradicts Proposition 2.8, which states that $\varphi\left(\boldsymbol{C}_{\boldsymbol{M}}\right) \iota\left(\boldsymbol{R}_{\boldsymbol{M}}\right)$ is left weighted.

As $M-t>0$, we have $\varphi\left(\boldsymbol{C}_{M}\right)=\varphi\left(X^{M-t}\right)$. Hence, $\varphi\left(X^{M-t}\right) \iota\left(X^{M-t}\right)=$ $\varphi\left(\boldsymbol{C}_{M}\right) \iota\left(\boldsymbol{R}_{M}\right)$ is left weighted, that is, $X^{M-t}$ is rigid and we can just choose $T=$ $M-t$.

We have then shown the following result.

Theorem 3.21. Let $X \in \operatorname{USS}(X)$ with $\ell(X)>1$. Then $X$ has a rigid power if and only if $C_{M}=\Delta^{k} X^{t}$ for some integers $M, k, t$, where $M, t>0$ and $\Delta^{k}$ is central.

Moreover, in this case $M-t>0$ and $M$ can be chosen so that $\boldsymbol{R}_{M}=\Delta^{s} X^{M-t}$ where $\Delta^{s}$ is central, and $X^{M}, X^{t}$ and $X^{M-t}$ are all rigid.

Proof. The first claim is shown in Propositions 3.18 and 3.20. In the proof of Proposition 3.20 it is also shown that in this case $M-t>0$. Replacing $M$ by some suitable multiples, we replace $X^{M}, X^{t}$ and $X^{M-t}$ by powers, hence we can choose $M$ in such a way that these three elements are rigid. 
A very interesting consequence of this result is the following

Theorem 3.22. Let $X \in \operatorname{USS}(X)$ with $\ell(X)>1$. If $X$ has a rigid power, then all elements in $\operatorname{USS}(X)$ have rigid powers.

Proof. By Theorem 3.21, $C_{M}=\Delta^{k} X^{t}$ for some integers $M, k, t$, where $M, t>0$ and $\Delta^{k}$ is central. We can also assume that $M$ is a multiple of the orbit length of $X$.

Let $Y \in \operatorname{USS}(X)$. For $i \geq 1$, let $C_{i}^{\prime}, R_{i}^{\prime}, \boldsymbol{C}_{i}^{\prime}$ and $\boldsymbol{R}_{i}^{\prime}$ denote the elements analogous to $C_{i}, R_{i}, C_{i}$ and $\boldsymbol{R}_{i}$, defined for $Y$ instead of $X$.

Let $\alpha$ be a positive element such that $\alpha^{-1} X \alpha=Y$. In [24], the $M$-th transport of $\alpha$ is defined as the element $\beta$ such that $\alpha^{-1} \boldsymbol{C}_{M} \beta=\boldsymbol{C}_{M}^{\prime}$. It is shown in [24] that some iterated transport of $\alpha$ will be equal to $\alpha$. Hence, replacing $M$ by a multiple if necessary, we can assume that $\beta=\alpha$. But then $\boldsymbol{C}_{M}^{\prime}=\alpha^{-1} \boldsymbol{C}_{M} \alpha=\alpha^{-1} \Delta^{k} X^{t} \alpha=$ $\Delta^{k} \alpha^{-1} X^{t} \alpha=\Delta^{k} Y^{t}$. By Theorem 3.21 this means that $Y$ also has a rigid power.

3.4. Consequences for pseudo-Anosov braids. The results from the previous subsection have a very important consequence in the case of braid groups. The structure of centralizers of pseudo-Anosov braids is well known, and this allows to show that pseudo-Anosov braids in their ultra summit set have rigid powers.

Theorem 3.23. Let $X \in B_{n}$ be a pseudo-Anosov braid. If $X \in \operatorname{USS}(X)$ and $\ell(X)>1$, then $X$ has a rigid power.

Proof. Let $N$ be the orbit length of $X$ under cycling. Then $C_{N}$ commutes with $X$. It is known [27], [26] that if $X$ is pseudo-Anosov, every element in the centralizer of $X$ has a common power with $X$, up to multiplication by a central power of $\Delta$. Hence $\left(\boldsymbol{C}_{N}\right)^{r}=\Delta^{k} X^{t}$ for some integers $r, k, t$, where $\Delta^{k}$ is central. Moreover we can assume that $r>0$, otherwise we consider the inverse of the above equation. Since $N$ is the orbit length of $X,\left(\boldsymbol{C}_{N}\right)^{r}=C_{N r}$, hence taking $M=N r$ one has $C_{M}=\Delta^{k} X^{t}$ for some positive $M$ and some integers $k, t$ such that $\Delta^{k}$ is central. By Theorem 3.21, we only need to show that $t>0$.

Suppose that $t<0$. Replacing $M$ by a multiple if necessary, we can assume that $M \geq\|\Delta\|$, hence $\varphi\left(\boldsymbol{C}_{\boldsymbol{M}}\right)=F(X)=\varphi\left(\boldsymbol{C}_{\boldsymbol{M r}}\right)=\varphi\left(X^{t r}\right)$ for every $r \geq 1$. We can also assume that $M$ is a (positive) multiple of $t$, hence $t-M$ will be a (negative) multiple of $t$, and then $\varphi\left(\boldsymbol{C}_{\boldsymbol{M}}\right)=\varphi\left(X^{t-\boldsymbol{M}}\right)$.

On the other hand, by Lemma 2.4, we know that $X^{M}=C_{M} \boldsymbol{R}_{M} \Delta^{M p}$, where $p=\inf (X)$. Hence $\boldsymbol{R}_{M}=X^{M-t} \Delta^{-k-M p}$, and then $\iota\left(\boldsymbol{R}_{M}\right)=\iota\left(X^{M-t}\right)=$ $\partial\left(\varphi\left(X^{t-M}\right)\right)$. This would contradict Proposition 2.8, which states that $\varphi\left(\boldsymbol{C}_{\boldsymbol{M}}\right) \iota\left(\boldsymbol{R}_{\boldsymbol{M}}\right)$ is left weighted. Therefore $t>0$, and Theorem 3.21 implies that $X$ has a rigid power.

Corollary 3.24. Every pseudo-Anosov braid has a rigid power, up to conjugacy. 
Proof. Let $Y$ be a pseudo-Anosov braid, and let $X \in \mathrm{SU}(Y)$. That is, $X$ is conjugate to $Y$ and all powers of $X$ belong to their ultra summit set.

We know that powers of $\Delta$ are not pseudo-Anosov but periodic, hence $\ell(X) \geq 1$. We will first show that we have $\ell\left(X^{m}\right)>1$ for some $m>1$. Indeed, if $\ell\left(X^{m}\right)=1$ for all $m>1$, since the set of simple elements is finite we would have $X^{a}=\Delta^{u} s$ and $X^{b}=\Delta^{v} s$ for the same simple element $s$ and $a \neq b$. But then $X^{b-a}=\Delta^{v-u}$, which is not possible since a pseudo-Anosov braid cannot be periodic.

Since the property of being pseudo-Anosov is preserved by powers, $X^{m}$ is a pseudo-Anosov braid such that $\ell\left(X^{m}\right)>1$. Moreover, $X^{m} \in \operatorname{USS}\left(X^{m}\right)$, as $X \in$ $\mathrm{SU}(Y)$. Hence we can apply Theorem 3.23 to $X^{m}$ and it follows that some power of $X^{m}$, thus some power of $X$, is rigid. Since $X$ is conjugate to $Y$, the result follows.

We remark that generic elements of $B_{n}$ are pseudo-Anosov. This means that most elements in $B_{n}$ have rigid powers, up to conjugacy.

3.5. A bound for the rigid power of an element. In this section we will show that if $X \in \operatorname{USS}(X)$ has a rigid power, and several powers of $X$ belong to their ultra summit sets, then $X^{m}$ is rigid for some small $m$, namely $m<\|\Delta\|^{3}$. Moreover, if $\ell(X)>1$ we can take $m<\|\Delta\|^{2}$. In the particular case of braid groups, using the Artin structure one has $\|\Delta\|=n(n-1) / 2$, and using the Birman-Ko-Lee structure $\|\Delta\|=n-1$. Hence in both cases the bound is polynomial on the number of strands, and does not depend on the length of the braid.

We first need to show two results concerning elements having rigid powers and absolute final factors.

Proposition 3.25. Let $X \in \operatorname{USS}(X)$ with $\ell(X)>1$. Suppose that $X$ has a rigid power, and that $X^{t} \in \operatorname{USS}\left(X^{t}\right)$ for some $t>1$. Then $F(X)=F\left(X^{t}\right)$.

Proof. By Theorem 3.21, one has $C_{M}=\Delta^{k} X^{r}$ for some $M, k, r$ such that $M, r>0$ and $\Delta^{k}$ is central. Replacing $M$ by a multiple if necessary, so that $M$ is a multiple of the orbit length of $X$ under cycling, one has $C_{[-M, M]}=C_{M}=\Delta^{k} X^{r}$.

In the same way, $X^{t}$ also has a rigid power. Moreover, since $\ell\left(C_{t}\right) \geq 1, \ell\left(\boldsymbol{R}_{t}\right) \geq 1$ and $\boldsymbol{C}_{t} \boldsymbol{R}_{t}$ is left weighted, one has $\ell\left(X^{t}\right)>1$. Hence we can apply Theorem 3.21 to $X^{t}$ and we obtain $C_{\left[-M^{\prime}, M^{\prime}\right]}^{(t)}=C_{M^{\prime}}^{(t)}=\Delta^{k^{\prime}} X^{t r^{\prime}}$ for some $M^{\prime}, r^{\prime}>0$ and some $k^{\prime}$ such that $\Delta^{k^{\prime}}$ is central.

Replacing $M$ and $M^{\prime}$ above by some suitable multiples, we can assume that $r=$ $t r^{\prime}$, and also that $M, M^{\prime} \geq\|\Delta\|$. Hence $F\left(X^{t}\right)=\varphi\left(\boldsymbol{C}_{\left[-M^{\prime}, M^{\prime}\right]}\right)=\varphi\left(\Delta^{k^{\prime}} X^{t r^{\prime}}\right)=$ $\varphi\left(\Delta^{k} X^{r}\right)=\varphi\left(C_{[-M, M]}\right)=F(X)$.

Proposition 3.26. Let $X \in \operatorname{USS}(X)$ with $\ell(X)>1$, and suppose that $X$ has a rigid power. If $\mathcal{R}(X)>0$, then $\iota(X)=I(X)$. If $\mathcal{R}\left(X^{-1}\right)>0$, then $\varphi(X)=F(X)$. 
Proof. By Theorem 3.21, there exists some $M>0$ such that $C_{M}=\Delta^{k} X^{t}$ and $\boldsymbol{R}_{M}=\Delta^{s} X^{M-t}$, where $t>0, M-t>0$, and both $\Delta^{k}$ and $\Delta^{s}$ are central.

Suppose that $\mathcal{R}\left(X^{-1}\right)>0$. By Corollary 3.6 this implies that $\varphi\left(X^{m}\right)=\varphi(X)$ for every $m \geq 1$. We can assume that $M$ is a multiple of the orbit length of $X$, hence $C_{[-M, M]}=C_{M}$. If one chooses $M$ big enough (replacing it by a multiple if necessary), one has $F(X)=\varphi\left(\boldsymbol{C}_{[-M, M]}\right)=\varphi\left(\boldsymbol{C}_{M}\right)=\varphi\left(X^{t}\right)=\varphi(X)$.

Now suppose that $\mathcal{R}(X)>0$. Then $\iota\left(X^{m}\right)=\iota(X)$ for every $m>0$, by Corollary 3.6. In the same way as above, since $M$ is a multiple of the orbit length of $X$ and $M-t>0$, replacing $M$ (and thus $t$ ) by a multiple if necessary one has $I(X)=\iota\left(\boldsymbol{R}_{[-M, M]}\right)=\iota\left(\boldsymbol{R}_{M}\right)=\iota\left(\Delta^{s} X^{M-t}\right)=\iota\left(X^{M-t}\right)=\iota(X)$.

In order to obtain the claimed bound on rigid powers, we need to investigate how the left normal form of $\boldsymbol{C}_{[k, m]}$ is modified when we multiply it on the left by $C_{k}$. We actually show the following, more general result.

Proposition 3.27. Let $X \in \operatorname{USS}(X)$ with $\ell(X)>1$, and suppose that $X$ has a rigid power. Let $t>1$ be such that $X^{t} \in \operatorname{USS}\left(X^{t}\right)$. Consider $C_{1} \ldots C_{m}=\Delta^{k} y_{1} \ldots y_{s}$ and $C_{m+1} \ldots C_{m+t}=\Delta^{q} z_{1} \ldots z_{r}$ in left normal form. Then, the final $t-1$ factors in the left normal form of $C_{1} \ldots C_{m+t}$ are precisely $z_{2} \ldots z_{r}$.

Proof. We will need to use the factors $C_{i}$ and $R_{i}$ corresponding to the element $Y=$ $\left(\mathbf{c}^{m}(X)\right)^{t}$. In order to avoid an excessive use of indices, we will denote them by $C_{i}^{\prime}$ and $R_{i}^{\prime}$. That is, $C_{1}^{\prime}=\iota(Y)$, and the other elements $C_{i}^{\prime}$ and $R_{i}^{\prime}$ are defined in the same way as the corresponding elements for $X$.

We know by Theorem 2.9 that $C_{m+1} \ldots C_{m+t}$ is equal to the product of the first $t$ factors (including $\Delta^{\prime}$ s) in the left normal form of $\left(\mathbf{c}^{m}(X)\right)^{t} \Delta^{-p t}$ (where $\left.p=\inf (X)\right)$. Hence $C_{1}^{\prime}=\iota\left(\left(\mathbf{c}^{m}(X)\right)^{t}\right)=\tau^{-q}\left(z_{1}\right)$, and $\tau^{-q}\left(z_{2}\right) \preccurlyeq R_{1}^{\prime}$.

Now one has

$$
\begin{aligned}
C_{1} \ldots C_{m} C_{m+1} \ldots C_{m+t} & =C_{1} \ldots C_{m} \Delta^{q} z_{1} \ldots z_{r} \\
& =C_{1} \ldots C_{m} \tau^{-q}\left(z_{1}\right) \tau^{-q}\left(z_{2}\right) \Delta^{q} z_{3} \ldots z_{r} .
\end{aligned}
$$

According to Proposition 2.12 and the definition of absolute final factors, one has $\varphi\left(C_{1} \ldots C_{m}\right) \succcurlyeq F\left(\mathbf{c}^{m}(X)\right)$. Recall from the proof of Proposition 2.23 that if $X^{t} \in$ $\mathrm{USS}\left(X^{t}\right)$ then $\left(\mathbf{c}^{m}(X)\right)^{t}$ also belongs to its ultra summit set, since the action of cycling or decycling any power of $X$ (in particular $X$ ) preserves the set of powers of $X$ belonging to their ultra summit set. Moreover, since $X$ has a rigid power, Theorem 3.22 implies that $\mathbf{c}^{m}(X)$ also has a rigid power. We can then apply Proposition 3.25 to obtain $F\left(\mathbf{c}^{m}(X)\right)=F\left(\left(\mathbf{c}^{m}(X)\right)^{t}\right)=F(Y)$. Hence $\varphi\left(C_{1} \ldots C_{m}\right) \succcurlyeq F(Y)$.

This yields the following:

$$
\varphi\left(C_{1} \ldots C_{m} \tau^{-q}\left(z_{1}\right)\right)=\varphi\left(C_{1} \ldots C_{m} C_{1}^{\prime}\right) \succcurlyeq \varphi\left(F(Y) C_{1}^{\prime}\right)=\varphi\left(C_{-a+1}^{\prime} \ldots C_{-1}^{\prime} C_{0}^{\prime} C_{1}^{\prime}\right)
$$


for $a$ big enough. But we know by Lemma 2.5 that the decomposition

$$
\varphi\left(C_{-a+1}^{\prime} \ldots C_{-1}^{\prime} C_{0}^{\prime} C_{1}^{\prime}\right) R_{1}^{\prime}
$$

is left weighted. Hence

$$
\varphi\left(C_{1} \ldots C_{m} \tau^{-q}\left(z_{1}\right)\right) \tau^{-q}\left(z_{2}\right)
$$

is also left weighted, and the factors $z_{2} \ldots z_{r}$ are not modified when computing the left normal form of $C_{1} \ldots C_{m+t}$.

The fact that the left normal forms of $\boldsymbol{C}_{[-m, m]}$ are not modified too much when one increases $m$, implies a strong property on the initial factors of powers of $X$ : they are comparable by $\preccurlyeq$. First we need the following technical result.

Lemma 3.28. Let $A=\Delta^{p} x_{1} \ldots x_{r} \in G$, and let $s$ be a simple element. Then either $\iota(A s) \preccurlyeq \iota(A)$ or $\iota(A) \preccurlyeq \iota(A s)$.

Proof. Suppose there is no unexpected $\Delta$ when multiplying $A$ by $s$, that is, $\inf (A s)=$ $p$. Then $\iota\left(\Delta^{p} x_{1} \ldots x_{r} s\right)=\tau^{-p}\left(x_{1} t\right)$ for some (possibly trivial) simple element $t$. Hence $\iota(A)=\tau^{-p}\left(x_{1}\right) \preccurlyeq \tau^{-p}\left(x_{1} t\right)=\iota(A s)$.

Now suppose there is an unexpected $\Delta$, that is, $\inf (A s)=p+1$. Let $\alpha=$ $\left(x_{2} \ldots x_{r} s\right) \wedge \Delta$. Then $\iota(A s)=\iota\left(\Delta^{p} x_{1} \ldots x_{r} s\right)=\iota\left(\Delta^{p} x_{1} \alpha\right)$. Moreover, $x_{1} \alpha=$ $\Delta \beta$ for some simple element $\beta$, that is, $\alpha=\partial\left(x_{1}\right) \beta$. Since $\alpha$ is simple, it follows that $\beta \preccurlyeq \partial^{2}\left(x_{1}\right)=\tau\left(x_{1}\right)$. Therefore

$$
\iota(A s)=\iota\left(\Delta^{p} x_{1} \alpha\right)=\iota\left(\Delta^{p+1} \beta\right) \preccurlyeq \iota\left(\Delta^{p+1} \tau\left(x_{1}\right)\right)=\tau^{-p}\left(x_{1}\right)=\iota(A) .
$$

Proposition 3.29. Let $X \in \operatorname{USS}(X)$ with $\ell(X)>1$. Suppose that $X$ has a rigid power, and let $t \geq 1$ such that $X^{t} \in \operatorname{USS}\left(X^{t}\right)$. Then for every $m \geq 1$, the simple elements $\iota\left(C_{1} \ldots C_{m}\right)$ and $\iota\left(C_{1} \ldots C_{m+t}\right)$ are comparable. That is, either

$$
\iota\left(C_{1} \ldots C_{m}\right) \preccurlyeq \iota\left(C_{1} \ldots C_{m+t}\right)
$$

or

$$
\iota\left(C_{1} \ldots C_{m+t}\right) \preccurlyeq \iota\left(C_{1} \ldots C_{m}\right) .
$$

Proof. Write $C_{1} \ldots C_{m}=\Delta^{k} y_{1} \ldots y_{s}$ and $C_{m+1} \ldots C_{m+t}=\Delta^{q} z_{1} \ldots z_{r}$. We know by Proposition 3.27 that

$$
\varphi\left(y_{1} \ldots y_{s} \tau^{-q}\left(z_{1}\right)\right) \tau^{-q}\left(z_{2}\right)
$$

is left weighted. Hence

$$
\iota\left(C_{1} \ldots C_{m+t}\right)=\iota\left(\Delta^{k} y_{1} \ldots y_{s} \tau^{-q}\left(z_{1}\right)\right),
$$

where $\tau^{-q}\left(z_{1}\right)$ is simple. By Lemma 3.28, this implies that $\iota\left(C_{1} \ldots C_{m+t}\right)$ is comparable to $\iota\left(\Delta^{k} y_{1} \ldots y_{s}\right)=\iota\left(C_{1} \ldots C_{m}\right)$, as we wanted to show. 
We can finally state the result concerning the initial factors of powers of $X$.

Corollary 3.30. Let $X \in \operatorname{USS}(X)$ with $\ell(X)>1$, and suppose that $X$ has a rigid power. If $X^{m} \in \operatorname{USS}\left(X^{m}\right)$ for $m=1, \ldots,\|\Delta\|$, then the set

$$
\left\{\iota(X), \iota\left(X^{2}\right), \ldots, \iota\left(X^{\|\Delta\|}\right)\right\}
$$

is totally ordered by $\preccurlyeq$ (although the total order given by $\preccurlyeq$ does not necessarily coincide with the above enumeration).

Proof. We just need to recall from Theorem 2.9 that $\iota\left(X^{m}\right)=\iota\left(C_{1} \ldots C_{m}\right)$ for every $m \geq 1$, and use the above result. Since every two elements are comparable by $\preccurlyeq$, the set is totally ordered.

Corollary 3.31. With the above conditions, there exist some integers $a, b$ with $1 \leq a<b \leq\|\Delta\|$ such that $\iota\left(X^{a}\right)=\iota\left(X^{b}\right)$.

Proof. The length of a strict chain of simple elements $1 \prec s_{1} \prec s_{2} \prec \cdots \prec s_{r} \prec \Delta$ is bounded by $\|\Delta\|$. Since the elements in $\left\{\iota(X), \iota\left(X^{2}\right), \ldots, \iota\left(X^{\|\Delta\|}\right)\right\}$ are totally ordered, the lack of a repeated pair would provide a chain of bigger length, which is not possible.

It is important to notice that, in the sequence $\iota(X), \iota\left(X^{2}\right), \iota\left(X^{3}\right), \ldots$, when one encounters the first repetition, the sequence becomes periodic. And the period is the distance between the two repeated elements. This is given by the following result.

Proposition 3.32. With the above conditions, if $\iota\left(X^{a}\right)=\iota\left(X^{b}\right)$ then $\iota\left(X^{a+k}\right)=$ $\iota\left(X^{b+k}\right)$ for every $k \geq 0$.

Proof. By hypothesis $\iota\left(C_{1} \ldots C_{a}\right)=\iota\left(X^{a}\right)=\iota\left(X^{b}\right)=\iota\left(C_{1} \ldots C_{b}\right)$. Applying to this equality the transport defined in [24], one obtains $\iota\left(C_{2} \ldots C_{a+1}\right)=$ $\iota\left(C_{2} \ldots C_{b+1}\right)$. Let $\iota=\iota\left(C_{2} \ldots C_{a+1}\right)=\iota\left(C_{2} \ldots C_{b+1}\right)$. By Proposition 3.27, if we multiply $C_{2} \ldots C_{a+1}$ or $C_{2} \ldots C_{b+1}$ on the left by $C_{1}$, only their initial factors (which in both cases are equal to $\iota$ ) are modified. Moreover, since $\sup \left(C_{1} \ldots C_{a+1}\right)=a+1$, it follows that $\sup \left(C_{1} \iota\right)=2$. Hence the initial factor of $C_{1} \ldots C_{a+1}$ is equal either to $\Delta \wedge\left(C_{1} \iota\right)$ (if the infimum does not increase) or to $\left(C_{1} \iota\right) \Delta^{-1}$ (if the infimum increases). In any case, $\iota\left(C_{1} \ldots C_{a+1}\right)=\iota\left(C_{1} \iota\right)$. In the same way, $\iota\left(C_{1} \ldots C_{b+1}\right)=\iota\left(C_{1} \iota\right)$, hence $\iota\left(X^{a+1}\right)=\iota\left(X^{b+1}\right)$. Induction on $k$ finishes the proof.

The above results can be used to bound the smallest power of $X$ having some rigidity.

Proposition 3.33. Let $X \in \mathrm{USS}(X)$ with $\ell(X)>1$, and suppose that $X$ has a rigid power. If $X^{t} \in \operatorname{USS}\left(X^{t}\right)$ for $t=1, \ldots,\|\Delta\|$, then $\mathcal{R}\left(X^{m}\right)>0$ for some positive $m<\|\Delta\|$. 
Proof. We know by Corollary 3.31 that $\iota\left(X^{a}\right)=\iota\left(X^{b}\right)$ for some $1 \leq a<b \leq\|\Delta\|$, and by Proposition 3.32 that the sequence $\iota\left(X^{a}\right), \iota\left(X^{a+1}\right), \iota\left(X^{a+2}\right), \ldots$ is periodic of period $d=b-a$.

Since the interval $[a, b]$ has length $d$, there exists a unique $m, a \leq m<b$, which is a multiple of $d$. Then $\iota\left(X^{m}\right)=\iota\left(X^{m+m}\right)=\iota\left(\left(X^{m}\right)^{2}\right)$. Hence, by Lemma 3.4, we will have $\mathcal{R}\left(X^{m}\right)>0$ if we show that $\inf \left(X^{2 m}\right)=2 \inf \left(X^{m}\right)$.

Suppose that $X^{m}=\Delta^{p} y_{1} \ldots y_{r}$. Since $m<b \leq\|\Delta\|$ one has $X^{m} \in \operatorname{USS}\left(X^{m}\right)$, so $\Delta \nless y_{2} \ldots y_{r} \tau^{-p}\left(y_{1}\right)$, which implies $\Delta \not y_{2} \ldots y_{r} \tau^{-p}\left(y_{1} \ldots y_{r}\right)$ since $\tau^{-p}\left(y_{1}\right)$ is the biggest simple prefix of $\tau^{-p}\left(y_{1} \ldots y_{r}\right)$. Then

$$
y_{1} \Delta \nless y_{1} y_{2} \ldots y_{r} \tau^{-p}\left(y_{1} \ldots y_{r}\right) .
$$

But if $\inf \left(X^{2 m}\right)=2 p+1$ then, since $X^{2 m}=\left(X^{m}\right)^{2}=\Delta^{p} y_{1} \ldots y_{r} \Delta^{p} y_{1} \ldots y_{r}$, we would have $\Delta \preccurlyeq \tau^{p}\left(y_{1} \ldots y_{r}\right) y_{1} \ldots y_{r}$, and since $\iota\left(X^{2 m}\right)=\iota\left(X^{m}\right)=\tau^{-p}\left(y_{1}\right)$, it would follow that $\Delta \tau^{p+1}\left(y_{1}\right) \preccurlyeq \tau^{p}\left(y_{1} \ldots y_{r}\right) y_{1} \ldots y_{r}$. Applying $\tau^{-p}$ to this inequality, we would obtain $\Delta \tau\left(y_{1}\right)=y_{1} \Delta \preccurlyeq y_{1} \ldots y_{r} \tau^{-p}\left(y_{1} \ldots y_{r}\right)$, a contradiction. Therefore $\inf \left(X^{2 m}\right)=2 p=\inf \left(X^{m}\right)$, and since $\iota\left(X^{2 m}\right)=\iota\left(X^{m}\right)$ it follows from Lemma 3.4 that $\mathcal{R}\left(X^{m}\right)>0$.

Theorem 3.34. Let $X \in \operatorname{USS}(X)$ with $\ell(X)>1$, and suppose that $X$ has a rigid power. If $X^{t} \in \operatorname{USS}\left(X^{t}\right)$ for every $t$ such that $-\|\Delta\| \leq t \leq\|\Delta\|$, then there is some $m<\|\Delta\|^{2}$ such that $X^{m}$ is rigid.

Proof. By Proposition 3.33, $\mathcal{R}\left(X^{p}\right)>0$ for some $0<p<\|\Delta\|$. Applying Proposition 3.33 to $X^{-1}$, one obtains that $\mathcal{R}\left(X^{-q}\right)>0$ for some $0<q<\|\Delta\|$.

Let $m=\operatorname{lcm}(p, q) \leq p q<\|\Delta\|^{2}$. Since $X^{m}$ is a power of $X^{p}$, it follows from Corollary 3.11 that $X^{m}$ belongs to a closed orbit under cycling, hence it has maximal infimum in its conjugacy class [20]. In the same way, since $X^{-m}$ is a power of $X^{-q}$, it follows from Corollary 3.11 that $X^{-m}$ belongs to a closed orbit under cycling, thus $X^{m}$ belongs to a closed orbit under decycling, and hence it has minimal supremum in its conjugacy class [20]. Therefore $X^{m} \in \operatorname{SSS}\left(X^{m}\right)$, and since it belongs to a closed orbit under cycling, $X^{m} \in \operatorname{USS}\left(X^{m}\right)$.

Moreover, by Proposition 3.9, $\mathcal{R}\left(X^{m}\right)>0$ and $\mathcal{R}\left(X^{-m}\right)>0$, since they are powers of $X^{p}$ and $X^{-q}$. This implies by Proposition 3.26 that $\iota\left(X^{m}\right)=I\left(X^{m}\right)$ and $\varphi\left(X^{m}\right)=F\left(X^{m}\right)$. Since $F\left(X^{m}\right) I\left(X^{m}\right)$ is left weighted by Proposition 2.20 , it follows that $\varphi\left(X^{m}\right) \iota\left(X^{m}\right)$ is left weighted as written, hence $X^{m}$ is rigid, as we wanted to show.

Remark. The proof of the above result is based on the fact that if an element $X$ is such that $\mathcal{R}(X)>0$ and $\mathcal{R}\left(X^{-1}\right)>0$, and if $X$ has a rigid power, then $X$ is already rigid. The hypothesis of $X$ having a rigid power is necessary, since we could have $X$ and $X^{-1}$ with some rigidity without $X$ being rigid, even if $X \in \mathrm{SU}(X)$. For instance, if we 
consider the reducible braid $X=\sigma_{1} \sigma_{3} \cdot \sigma_{3} \in B_{4}$, we have $\mathcal{R}(X)=\mathcal{R}\left(X^{-1}\right)=1 / 2$, but neither $X$ nor any power of $X$ is rigid, since $X^{m}=\left(\sigma_{1} \sigma_{3}\right)^{m} \cdot \sigma_{3}^{m}$. In this case $X \in \mathrm{SU}(X)$ and $\mathcal{R}\left(X^{m}\right)=1 / 2$ for every $m \neq 0$.

We can also find a bound for the smallest rigid power in the case $\ell(X)=1$, thanks to the following result.

Lemma 3.35. Let $X \in G$ with $\ell(X)=1$. If $X^{t} \in \operatorname{USS}\left(X^{t}\right)$ for $t=1, \ldots,\|\Delta\|$, then $\ell\left(X^{m}\right) \neq 1$ for some $m \leq\|\Delta\|$.

Proof. Suppose that $\ell\left(X^{t}\right)=1$ for $t=1, \ldots,\|\Delta\|$. We will show that the set $\left\{\iota(X), \iota\left(X^{2}\right), \ldots, \iota\left(X^{\|\Delta\|}\right)\right\}$ is totally ordered by showing that any two elements in that set are comparable. Indeed, given $s, t \in\{1, \ldots,\|\Delta\|\}$ with $s<t$, we have $\ell\left(X^{t-s}\right)=1$, hence $\iota\left(X^{t}\right)=\iota\left(X^{s} X^{t-s}\right)=\iota\left(X^{s} \iota\left(X^{t-s}\right)\right)$. Since $\iota\left(X^{t-s}\right)$ is simple, Lemma 3.28 implies that either $\iota\left(X^{t}\right) \preccurlyeq \iota\left(X^{s}\right)$ or $\iota\left(X^{s}\right) \preccurlyeq \iota\left(X^{t}\right)$. Therefore, $\left\{\iota(X), \iota\left(X^{2}\right), \ldots, \iota\left(X^{\|\Delta\|}\right)\right\}$ is a totally ordered set of proper simple elements, thus $\iota\left(X^{a}\right)=\iota\left(X^{b}\right)$ for some $1 \leq a<b \leq\|\Delta\|$. But since $\ell\left(X^{a}\right)=\ell\left(X^{b}\right)=1$, this means that $X^{b-a}$ is a power of $\Delta$, a contradiction. Therefore, $\ell\left(X^{m}\right) \neq 1$ for some $m \leq\|\Delta\|$.

We can finally remove the hypothesis $\ell(X)>1$ in order to bound the rigid power of an element.

Theorem 3.36. Let $X \in \operatorname{USS}(X)$, and suppose that $X$ has a rigid power. If $X^{t} \in$ $\mathrm{USS}\left(X^{t}\right)$ for every $t$ such that $-\|\Delta\|^{2} \leq t \leq\|\Delta\|^{2}$, then there is some $m<\|\Delta\|^{3}$ such that $X^{m}$ is rigid.

Proof. If $\ell(X)>1$ the result follows from Theorem 3.34. If $\ell(X)=1$, Lemma 3.35 implies that $\ell\left(X^{r}\right) \neq 1$ for some $r \leq\|\Delta\|$. We cannot have $\ell\left(X^{r}\right)=0$, otherwise $X$ would be periodic and would not have a rigid power. Hence $\ell\left(X^{r}\right)>1$, and $\left(X^{r}\right)^{t} \in \operatorname{USS}\left(\left(X^{r}\right)^{t}\right)$ for $-\|\Delta\| \leq t \leq\|\Delta\|$. The hypothesis of Theorem 3.34 are then satisfied by $X^{r}$, hence $\left(X^{r}\right)^{s}$ is rigid for some $s<\|\Delta\|^{2}$. Therefore $X^{r s}$ is rigid with $r s<\|\Delta\|^{3}$.

In the case of braid groups, the above result implies the following.

Theorem 3.37. If $X \in B_{n}$ is a pseudo-Anosov braid, then $\operatorname{USS}\left(X^{m}\right)$ consists of rigid braids, for some $m<\|\Delta\|^{3}$. Moreover, if the canonical length of the elements in $\mathrm{USS}(X)$ is greater than 1 , then $m<\|\Delta\|^{2}$.

Proof. By Corollary 3.24, some conjugate $Y$ of $X$ has a rigid power. Moreover, one can choose $Y \in \mathrm{SU}(X)$, hence by Theorem $3.36 Y^{m}$ is rigid for $m<\|\Delta\|^{3}$ (and $m<\|\Delta\|^{2}$ if $\ell(Y)>1$ ). In the proof of Theorem 3.36 we see that we can assume 
$\ell\left(Y^{m}\right)>1$, hence it follows from Theorem 3.15 that $\operatorname{USS}\left(Y^{m}\right)$ consists of rigid elements.

To summarize the consequences for pseudo-Anosov braids, we can solve the CDP/CSP problem for two pseudo-Anosov elements $X, Y \in B_{n}$ using rigid braids. We just need to compute an element in $\operatorname{USS}\left(X^{t}\right)$ for each $t=1,2, \ldots$ until we find one of them, say $\tilde{X}^{m} \in \operatorname{USS}\left(X^{m}\right)$ which is rigid and has canonical length greater than one. By the above result, $m<\|\Delta\|^{3}$. Then all elements in $\operatorname{USS}\left(X^{m}\right)$ will be rigid, so the computation of $\operatorname{USS}\left(X^{m}\right)$ is easier than in the general case, as will be seen in [9], and we will possibly be able to bound the size of $\operatorname{USS}\left(X^{m}\right)$. Moreover, since pseudo-Anosov braids have unique roots, if one solves the CDP/CSP for $X^{m}$ and $Y^{m}$, finding some conjugating element $Z$, then $Z$ is also a conjugating element for $X$ and $Y$, so this solves the CDP/CSP for $X$ and $Y$.

\section{References}

[1] S. I. Adyan, Fragments of the word $\Delta$ in the braid group. Mat. Zametki 36 (1984), 25-34; English transl. Math. Notes 36 (1984), 505-510. Zbl 0599.20044 MR 757642

[2] I. Anshel, M. Anshel and D. Goldfeld, An algebraic method for public-key cryptography. Math. Res. Lett. 6 (1999), 287-291. Zbl 0944.94012 MR 1713130

[3] E. Artin, Theorie der Zöpfe. Abh. Math. Sem. Univ. Hamburg 4 (1925), 47-72. JFM 51.0450.01

[4] D. Benardete, M. Gutiérrez, and Z. Nitecki, A combinatorial approach to reducibility of mapping classes. In Mapping class groups and moduli spaces of Riemann surfaces (Göttingen, 1991/Seattle, WA, 1991), Contemp. Math. 150, Amer. Math. Soc., Providence, RI, 1993, 1-31. Zbl 0804.57005 MR 1234257

[5] D. Bernardete, Z. Nitecki, and M. Gutiérrez, Braids and the Nielsen-Thurston classification. J. Knot Theory Ramifications 4 (1995), 549-618. Zbl 0874.57010 MR 1361083

[6] D. Bessis, The dual braid monoid. Ann. Sci. École Norm. Sup. (4) 36 (2003), 647-683. Zbl 1064.20039 MR 2032983

[7] D. Bessis, F. Digne, and J. Michel, Springer theory in braid groups and the Birman-Ko-Lee monoid. Pacific J. Math. 205 (2002), 287-309. Zbl 1056.20023 MR 1922736

[8] M. Bestvina, M. Handel, Train-tracks for surface homeomorphisms. Topology 34 (1995), 109-140. Zbl 0837.57010 MR 1308491

[9] J. S. Birman, V. Gebhardt, and J. González-Meneses, Conjugacy in Garside groups II: structure of the ultra summit set. Groups Geom. Dyn., to appear; preprint 2006, arXiv:math.GT/0606652

[10] J. S. Birman, V. Gebhardt, and J. González-Meneses, Conjugacy in Garside groups III: periodic braids. J. Algebra, to appear; preprint 2006, arXiv:math.GT/0609616

[11] J. S. Birman, K. H. Ko, and S. J. Lee, A new approach to the word and conjugacy problems in the braid groups. Adv. Math. 139 (1998), 322-353. Zbl 0937.20016 MR 1654165 
[12] J. S. Birman, K. H. Ko, and S. J. Lee, The infimum, supremum, and geodesic length of a braid conjugacy class. Adv. Math. 164 (2001), 41-56. Zbl 1063.20039 MR 1870512

[13] N. Bourbaki, Groupes et algèbres de Lie, Chapitres IV, V et VI: Groupes de Coxeter et systèmes de Tits, groupes engendrés par des réflexions, systèmes de racines. Hermann, Paris 1968. Zbl 0186.33001 MR 0240238

[14] E. Brieskorn and K. Saito, Artin-Gruppen und Coxeter-Gruppen. Invent. Math. 17 (1972), 245-271. Zbl 0243.20037 MR 0323910

[15] J. Crisp, private communication.

[16] P. Dehornoy, Groupes de Garside. Ann. Sci. École Norm. Sup. (4) 35 (2002), 267-306. Zbl 1017.20031 MR 1914933

[17] P. Dehornoy and L. Paris, Gaussian groups and Garside groups, two generalisations of Artin groups. Proc. London Math. Soc. (3) 79 (1999), 569-604. Zbl 1030.20021 MR 1710165

[18] P. Deligne, Les immeubles des groupes de tresses généralisés. Invent. Math 17 (1972), 273-302. Zbl 0238.20034 MR 0422673

[19] I. Dynnikov and B. Wiest, On the complexity of braids. J. Eur. Math. Soc., to appear; preprint 2004, arXiv:math.GT/0403177

[20] E. A. Elrifai and H. R. Morton, Algorithms for positive braids. Quart. J. Math. Oxford Ser. (2) 45 (1994), 479-497. Zbl 0839.20051 MR 1315459

[21] D. B. A. Epstein, J. W. Cannon, D. F. Holt, S. V. F. Levy, M. S. Paterson, and W. P. Thurston, Word processing in groups. Jones and Bartlett Publishers, Boston 1992. Zbl 0764.20017 MR 1161694

[22] N. Franco and J. González-Meneses, Conjugacy problem for braid groups and Garside groups. J. Algebra 266 (2003), 112-132. Zbl 1043.20019 MR 1994532

[23] F. Garside, The braid group and other groups. Quart. J. Math. Oxford Ser. (2) 20 (1969), 235-254. Zbl 0194.03303 MR 0248801

[24] V. Gebhardt, A new approach to the conjugacy problem in Garside groups. J. Algebra 292 (2005), 282-302. Zbl 1105.20032 MR 2166805

[25] J. González-Meneses, The $n$th root of a braid is unique up to conjugacy. Algebr. Geom. Topol. 3 (2003), 1103-1118. Zbl 1063.20041 MR 2012967

[26] J. González-Meneses and B. Wiest, On the structure of the centralizer of a braid. Ann. Sci. École Norm. Sup. (4) 37 (2004), 729-757. Zbl 1082.20024 MR 2103472

[27] N. V. Ivanov, Subgroups of Teichmüller modular groups. Transl. Math. Monogr. 115, Amer. Math. Soc., Providence, RI, 1992. Zbl 0776.57001 MR

[28] K. H. Ko, S. J. Lee, J. H. Cheon, J. W. Han, J.-s. Kang, and C. Park, New public-key cryptosystem using braid groups. In Advances in cryptology - CRYPTO 2000 (Santa Barbara, CA), Lecture Notes in Comput. Sci. 1880, Springer, Berlin 2000, 166-183. Zbl 0995.94531 MR 1850042

[29] E.-K. Lee and S. J. Lee, A Garside-theoretic approach to the reducibility problem in braid groups. Preprint 2005, arXiv:math.GT/0506188. 
[30] E. K. Lee and S. J. Lee, Abelian subgroups of Garside groups. Comm. Algebra, to appear; preprint 2006, arXiv:math.GT/0609683.

[31] E. K. Lee and S. J. Lee, Translation numbers in a Garside group are rational with uniformly bounded denominators. J. Pure Appl. Algebra, to appear; preprint 2006, arXiv:math.GT/0604061

[32] E. K. Lee and S. J. Lee, Some power of an element in a Garside group is conjugate to a periodically geodesic element. Bull. Lond. Math. Soc., to appear; preprint 2006, arXiv:math.GN/0604144

[33] S. J. Lee, Garside groups are strongly translation discrete. J. Algebra 309 (2007), 594-609.

[34] J. Michel, A note on words in braid monoids. J. Algebra 215 (1999), 366-377. Zbl 0937.20017 MR 1684142

[35] J. Nielsen, Investigations in the topology of closed orientable surfaces I, II, III. In Collected mathematical papers, vol. 1, Birkhäuser, Boston 1986, 223-341; 348-400; 401-458. Zbl 0609.01050 MR 0865335

[36] M. Picantin, Petits Groupes Gaussiens. PhD thesis, Université de Caen, Caen 2000; http://www.liafa.jussieu.fr/ picantin/publi.html

[37] M. Picantin, The conjugacy problem in small Gaussian groups. Comm. Algebra 29 (2001), 1021-1039. Zbl 0988.20024 MR 1842395

[38] W. Thurston, On the geometry and dynamics of diffeomorphisms of surfaces Bull. Amer. Math. Soc. (N.S.) 19 (1988), 417-431. Zbl 0674.57008 MR 956596

[39] P. J. Xu, The genus of closed 3-braids. J. Knot Theory Ramifications 1 (1992), 303-326. Zbl 0773.57007 MR 1180404

Received September 29, 2006; revised March 20, 2007

J. S. Birman, Department of Mathematics, Barnard College and Columbia University, 2990 Broadway, New York, New York 10027, U.S.A.

E-mail: jb@math.columbia.edu

V. Gebhardt, School of Computing and Mathematics, University of Western Sydney,

Locked Bag 1797, Penrith South DC NSW 1797, Australia

E-mail: v.gebhardt@uws.edu.au

J. González-Meneses, Departamento de Álgebra, Universidad de Sevilla, Apdo. 1160, 41080 Sevilla, Spain

E-mail: meneses@us.es 